\title{
Silver-Catalyzed N-H Functionalization of Aryl/Aryl Diazoalkanes with Anilines
}

\author{
Feifei He, ${ }^{\#}$ Claire Empel ${ }^{\#}$ and Rene M. Koenigs* \\ Institute of Organic Chemistry, RWTH Aachen University, \\ Landoltweg 1, D-52074 Aachen, Germany \\ e-mail: rene.koenigs@rwth-aachen.de
}

Supporting Information

\section{Table of Contents}

$\begin{array}{ll}\text { General Information } & \text { S1 }\end{array}$

$\begin{array}{ll}\text { Important Safety Note } & \text { S1 }\end{array}$

Source and Preparation of Starting Materials $\quad$ S2

General Procedures $\quad$ S3

Reaction Optimization $\quad$ S4

Physical Data $\quad$ S5

$\begin{array}{lr}\text { References } & \text { S22 }\end{array}$

$\begin{array}{lr}\text { Spectra } & \text { S23 }\end{array}$ 


\section{General Information}

Unless otherwise noted, all commercially available compounds were used as provided without further purification. Chemicals used in this manuscript were purchased from Sigma Aldrich, Alfa Aesar, Fluorochem and Carl Roth.

Solvents used in reactions were p.A. grade. All reactions were performed under argon using degassed solvents. Solvents for chromatography were technical grade and distilled prior to use. Analytical thin-layer chromatography (TLC) was performed on Macherey-Nagel silica gel aluminium plates with F-254 indicator, visualised by irradiation with UV light. Column chromatography was performed using silica gel Merck 60 (particle size $0.063-0.2 \mathrm{~mm}$ ). Solvent mixtures are understood as volume/volume.

${ }^{1} \mathrm{H}$ NMR, ${ }^{19} \mathrm{~F}$ NMR and ${ }^{13} \mathrm{C}$ NMR were recorded on a Varian AV600/AV400 or an Agilent DD2 $400 \mathrm{NMR}$ spectrometer in $\mathrm{CDCl}_{3}$. Data are reported in the following order: chemical shift $(\delta)$ in ppm; multiplicities are indicated br (broadened singlet), s (singlet), d (doublet), t (triplet), q (quartet), m (multiplet); coupling constants $(J)$ are in Hertz (Hz).

HRMS data were recorded on a ThermoFisher Scientific LTQ Orbitrap XL using ESI ionization or on a Finnigan MAT 95 using EI ionization at $70 \mathrm{eV}$.

IR spectra were recorded on a Perkin Elmer-100 spectrometer and are reported in terms of frequency of absorption $\left(\mathrm{cm}^{-1}\right)$. Blue LEDs used in this manuscript were Kessil PR160L, 467 $\mathrm{nm}$. Reactions were irradiated from $4 \mathrm{~cm}$, temperature was set to ambient and cooling was realized with a fan.

\section{Important safety note}

Handling of diazo compounds should only be done in a well-ventilated fume cupboard using an additional blast shield. No incidents occurred when handling diazoalkanes, yet the reader should be aware of carcinogenicity and explosiveness of the herein described diazo compounds. General safety precautions when working with diazomethane and its derivatives should be followed. Any reactions described in this manuscript should not be performed without strict risk assessment and proper safety precautions. 


\section{Source and Preparation of Staring Materials}

\section{General Procedure for Starting Material Synthesis}<smiles>[R][X]c1ccc(C(=O)c2ccccc2)cc1</smiles>

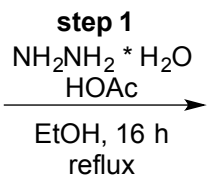<smiles>C[R]1cccc(C(=N)c2cc[R]cc2)c1</smiles>

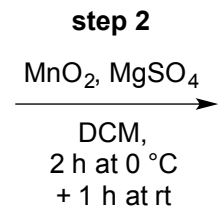<smiles>[R][R]1ccc(C(=N)c2ccc([R])cc2)cc1</smiles>

The diary diazo compounds were prepared in accordance to a modified procedure by Zhou et $a l .{ }^{[1]}$ In a first step, the corresponding benzophenone $(5 \mathrm{mmol})$ was dissolved in $30 \mathrm{~mL} \mathrm{EtOH}$, followed by the addition of hydrazine monohydrate $(50 \mathrm{mmol})$ and $0.5 \mathrm{~mL}$ concentrated acetic acid. The resulting mixture was refluxed for $16 \mathrm{~h}$ overnight. After cooling to room temperature, the corresponding hydrazone participated as crystals and was filtered. In a second step, the crude hydrazone was dissolved in $30 \mathrm{~mL}$ DCM at $0{ }^{\circ} \mathrm{C}$. To the stirring solution activated $\mathrm{MgO}_{2}$ ( $25 \mathrm{mmol})$ and $\mathrm{MgSO}_{4}(6 \mathrm{mmol})$ were added at once. The reaction mixture was stirred at $0{ }^{\circ} \mathrm{C}$ for $2 \mathrm{~h}$ and for an additional $1 \mathrm{~h}$ at room temperature. After filtration, the solvent was removed under reduced pressure and the product was purified by silica column chromatography using $n$-pentane : $\mathrm{Et}_{3} \mathrm{~N}$ as eluent. All diaryldiazo compounds were isolated as solids or liquids.

Note: Diaryldiazo compounds are light-sensitive; to avoid thermal decomposition, they should be stored in the freezer and should be used within 4 weeks after preparation.<smiles>[R]c1ccc(C(=N)c2ccc(Br)cc2)cc1</smiles>

$1 \mathrm{a}, \mathrm{R}=\mathrm{R}^{\prime}=\mathrm{H}$, ref. 2 1b, $R=O M e, R^{\prime}=O M e$, ref. 3 1c, $\mathrm{R}=\mathrm{Cl}, \mathrm{R}^{\prime}=\mathrm{OMe}$, ref. 1<smiles>N#CC(c1ccccc1)c1ccc(-c2ccccc2)cc1</smiles>

1d, ref. 3<smiles>Cc1cccc(C(=N)c2ccccc2)c1</smiles>

$1 e$,

characterized see page S5<smiles>Cc1ccccc1C(=N)c1ccccc1</smiles>

1f, ref. 4<smiles>N#CC(c1ccccc1)c1ccccc1Cl</smiles>

$1 \mathrm{~g}$, ref. 4<smiles>COc1ccc(C(=N)c2cccc([N+](=O)[O-])c2)cc1</smiles>

1h, characterized see page S5<smiles>COc1ccc(C(=N)c2ccc([N+](=O)[O-])cc2)cc1</smiles>

1i, ref. 2<smiles>COc1ccc(C(=N)c2cc(C)ccc2C)cc1</smiles>

1j,

characterized see page S6

\section{Sources of Staring Materials and Silver Catalysts}

\begin{tabular}{|l|l|l|}
\hline Supplier & Staring Materials & Silver Catalysts \\
\hline abcr & $\mathbf{9 j}, \mathbf{1 3 f}$ & \\
\hline Acros organics & $\mathbf{9 b}, \mathbf{9 m}, \mathbf{9 n}, \mathbf{1 1 b}, \mathbf{1 3 a}, \mathbf{1 3 e}$ & \\
\hline Alfa aeser & $\mathbf{9 d}, \mathbf{9 h}, \mathbf{9 k}, \mathbf{9 0}, \mathbf{9 q}, \mathbf{1 1 a}, \mathbf{1 1 g}, \mathbf{1 3 d}$ & $\mathrm{AgNTf}_{2}, \mathrm{AgOTf}_{\mathrm{AgPF}}, \mathrm{AgSbF}_{6}$ \\
\hline Fluka & $\mathbf{9 a}$ & \\
\hline Fluorochem & $\mathbf{9 g}, \mathbf{9 1}, \mathbf{1 1 e}, \mathbf{1 1}$ & \\
\hline Sigma Aldrich & $\mathbf{9 c}, \mathbf{9 e}, \mathbf{9 f}, \mathbf{9 i}, \mathbf{9 p}, \mathbf{1 1 c}, \mathbf{1 1 d}, \mathbf{1 3 b}$ & $\mathrm{AgBF}_{4}$ \\
\hline TCI & $\mathbf{1 3 c}$ & \\
\hline
\end{tabular}




\section{General Procedures}

\section{General Procedure for N-H insertion reaction of primary anilines (GP-1)}

In an oven-dried test tube, $\mathrm{AgPF}_{6}(3 \mathrm{~mol} \%)$ was put under vacuum and flushed with argon for three times. Dry, degassed DCM $(0.5 \mathrm{~mL})$ was added. Aniline 9 and aryl $/$ aryl diazo $1(0.2 \mathrm{mmol}$, 1.0 eq.) were dissolved in $0.5 \mathrm{~mL}$ of dry, degassed DCM and was added to the reaction mixture in one portion. The reaction mixture was stirred for 10 mins at room temperature. The product was purified by silica column chromatography using $n$-hexane: ethyl acetate as eluent.

\section{General Procedure for $\mathrm{N}-\mathrm{H}$ insertion reaction of secondary anilines (GP-2)}

In an oven-dried test tube $\mathrm{AgPF}_{6}(3 \mathrm{~mol} \%)$ was put under vacuum and flushed with argon for three times. Dry, degassed DCM $(0.5 \mathrm{~mL})$ was added. Aniline 11 and aryl/aryl diazo $1(0.2$ mmol, 1.0 eq.) were dissolved in $0.5 \mathrm{~mL}$ of dry, degassed DCM and was added to the reaction mixture over the period of $24 \mathrm{~h}$ using a syringe pump. The reaction mixture was stirred for additional $1 \mathrm{~h}$ at room temperature. The product was purified by silica column chromatography using $n$-hexane: ethyl acetate as eluent.

\section{1 mmol Scale Experiment}

In an oven-dried test tube, $\mathrm{AgPF}_{6}(3 \mathrm{~mol} \%, 7.58 \mathrm{mg})$ was put under vacuum and flushed with argon for three times. Dry, degassed DCM $(5.0 \mathrm{~mL})$ was added. Aniline 9a $(93.1 \mathrm{mg}, 1.0 \mathrm{mmol}$, 1.0 eq.) and aryl/aryl diazo $1 \mathrm{a}(194 \mathrm{mg}, 1.0 \mathrm{mmol}, 1.0$ eq.) were dissolved in $5.0 \mathrm{~mL}$ of dry, degassed DCM and was added to the reaction mixture in one portion. The reaction mixture was stirred for 10 mins at room temperature. The product was purified by silica column chromatography using $n$-hexane: ethyl acetate $80: 1 \rightarrow 40: 1$ as eluent and was obtained as a colorless oil in $78 \%$ yield $(203.3 \mathrm{mg})$. 


\section{Reaction Optimization}

\section{Reaction Optimization for Primary Anilines}

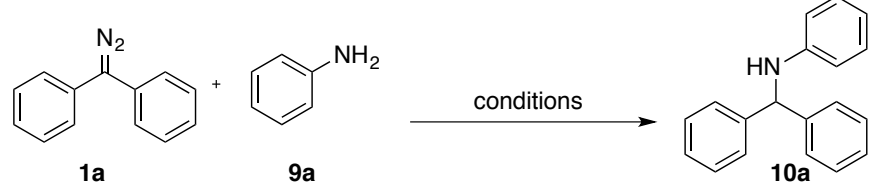

\begin{tabular}{|c|c|c|c|c|c|c|}
\hline Entry $^{[a]}$ & Catalyst & Additive & Solvent & Time & Eq. 1a:9a & Yield 10a $a^{[b]}$ \\
\hline 1 & $\begin{array}{l}\text { JohnPhos }(\mathrm{AuCl}) \\
(3 \mathrm{~mol} \%)\end{array}$ & $\begin{array}{l}\mathrm{AgSbF}_{6} \\
\left(5 \mathrm{~mol}^{2}\right)\end{array}$ & DCM & $10 \mathrm{~min}$ & $1: 2$ & $73 \%$ \\
\hline 2 & $\begin{array}{l}\text { JohnPhos }(\mathrm{AuCl}) \\
(3 \mathrm{~mol} \%)\end{array}$ & $\begin{array}{l}\mathrm{NaBAr}_{4}{ }^{\mathrm{F}} \\
(5 \mathrm{~mol} \%)\end{array}$ & DCM & $10 \mathrm{~min}$ & $1: 2$ & $20 \%$ \\
\hline 3 & $\mathrm{PdCl}_{2}(3 \mathrm{~mol} \%)$ & - & DCM & $24 \mathrm{~h}$ & $1: 2$ & trace \\
\hline 4 & CuOTf (3 mol\%) & - & DCM & $2 \min$ & $1: 2$ & $29 \%$ \\
\hline 5 & $\begin{array}{l}{\left[\mathrm{Ru}(p \text {-cymene }) \mathrm{Cl}_{2}\right]_{2}} \\
(3 \mathrm{~mol} \%)\end{array}$ & - & DCM & $24 \mathrm{~h}$ & $1: 2$ & no rct. \\
\hline 6 & $\mathrm{Rh}_{2}(\mathrm{OAc})_{4}$ & - & DCM & $24 \mathrm{~h}$ & $1: 2$ & no rct. \\
\hline 7 & $\mathrm{~B}\left(\mathrm{C}_{6} \mathrm{~F}_{5}\right)_{3}$ & - & DCM & $16 \mathrm{~h}$ & $1: 2$ & $15 \%$ \\
\hline 8 & $\operatorname{AgSbF}_{6}(3 \mathrm{~mol} \%)$ & - & DCM & $10 \mathrm{~min}$ & $1: 2$ & $74 \%$ \\
\hline 9 & $\mathrm{AgPF}_{6}(3 \mathrm{~mol} \%)$ & - & DCM & $10 \mathrm{~min}$ & $1: 2$ & $84 \%$ \\
\hline 10 & $\operatorname{AgNTf}_{2}(3 \mathrm{~mol} \%)$ & - & DCM & $10 \mathrm{~min}$ & $1: 2$ & $79 \%$ \\
\hline 11 & $\mathrm{AgBF}_{4}(3 \mathrm{~mol} \%)$ & - & DCM & $10 \mathrm{~min}$ & $1: 2$ & $83 \%$ \\
\hline 12 & $\operatorname{AgOTf}(3 \mathrm{~mol} \%)$ & - & DCM & $10 \mathrm{~min}$ & $1: 2$ & $78 \%$ \\
\hline 13 & $\mathrm{AgPF}_{6}(3 \mathrm{~mol} \%)$ & - & $\mathrm{THF}$ & $10 \mathrm{~min}$ & $1: 2$ & $67 \%$ \\
\hline 14 & $\mathrm{AgPF}_{6}(3 \mathrm{~mol} \%)$ & - & $\mathrm{MeCN}$ & $10 \mathrm{~min}$ & $1: 2$ & $58 \%$ \\
\hline 15 & $\mathrm{AgPF}_{6}(3 \mathrm{~mol} \%)$ & - & toluene & $10 \mathrm{~min}$ & $1: 2$ & $60 \%$ \\
\hline 16 & $\mathrm{AgPF}_{6}(3 \mathrm{~mol} \%)$ & - & 1,2-DCE & $10 \mathrm{~min}$ & $1: 2$ & $72 \%$ \\
\hline 17 & $\mathrm{AgPF}_{6}(3 \mathrm{~mol} \%)$ & - & DCM & $10 \mathrm{~min}$ & $1: 1$ & $86 \%$ \\
\hline 18 & $\mathrm{AgPF}_{6}(3 \mathrm{~mol} \%)$ & - & DCM & $10 \mathrm{~min}$ & $2: 1$ & $74 \%$ \\
\hline 19 & $\mathrm{AgPF}_{6}(6 \mathrm{~mol} \%)$ & - & DCM & $10 \mathrm{~min}$ & $1: 1$ & $81 \%$ \\
\hline 20 & $\mathrm{AgPF}_{6}(10 \mathrm{~mol} \%)$ & - & $\mathrm{DCM}$ & $10 \mathrm{~min}$ & $1: 1$ & $78 \%$ \\
\hline 21 & $\mathrm{HPF}_{6}(3 \mathrm{~mol} \%)$ & - & DCM & $24 \mathrm{~h}$ & $1: 2$ & no rct. \\
\hline 22 & - & - & DCM & $24 \mathrm{~h}$ & $1: 2$ & no rct. \\
\hline 23 & blue LEDs & - & DCM & $30 \mathrm{~min}$ & $1: 2$ & $35 \%$ \\
\hline
\end{tabular}

[a] reaction conditions: In an oven-dried test tube, catalyst and additive were out under vacuum and flushed with argon for three times. Dry, degassed solvent indicated $(0.5 \mathrm{~mL})$ was added. Aniline and aryl/aryl-diazo was dissolved in $0.5 \mathrm{~mL}$ of dry, degassed solvent indicated and was added to the reaction mixture in one portion. The reaction mixture was stirred at room temperature until the aryl/aryl diazo was consumed. [b] isolated yield. 


\section{Physical Data}

\section{1-(Diazo(phenyl)methyl)-3-methylbenzene (1e)}<smiles>Cc1cccc(C(=N)c2ccccc2)c1</smiles>

Compound 1e was prepared according to the general procedure for starting material synthesis on a $5 \mathrm{mmol}$ scale and was obtained after silica column chromatography ( $n$-pentane : $\mathrm{Et}_{3} \mathrm{~N}$ 1:1 $\rightarrow 20: 1) ; 54 \%$, (561 mg); red liquid.

${ }^{1}$ H NMR (600 MHz, Chloroform-d): $\delta={ }^{1} \mathrm{H}$ NMR (600 MHz, Chloroform-d) $\delta 7.50-7.43$ (m, $2 \mathrm{H}), 7.38-7.32(\mathrm{~m}, 3 \mathrm{H}), 7.29-7.22(\mathrm{~m}, 1 \mathrm{H}), 7.21-7.18(\mathrm{~m}, 2 \mathrm{H}), 7.12-7.06(\mathrm{~m}, 1 \mathrm{H}), 2.43$ (s, 3H) ppm.

${ }^{13}$ C NMR (151 MHz, Chloroform-d): $\delta=138.9,129.7,129.4,129.1,129.0,126.6,125.8,125.5$, $125.2,122.4,21.6 \mathrm{ppm}$.

HRMS (EI): $m / z$ : [M] ${ }^{+}$Calcd. for $\mathrm{C}_{14} \mathrm{H}_{12} \mathrm{~N}_{2}$ : 208.09950; Found: 208.09920 .

IR (KBr): 3574, 3029, 2919, 2863, 2723, 2328, 2163, 2034, 1944, 1873, 1799, 1660, 1594, $1491,1447,1378,1305,1267,1174,1078,1033,963,891,830,778,751,691 \mathrm{~cm}^{-1}$.

\section{1-(Diazo(4-methoxyphenyl)methyl)-3-nitrobenzene (1h)}<smiles>COc1ccc(C(=N)c2cccc([N+](=O)[O-])c2)cc1</smiles>

Compound $\mathbf{1} \mathbf{j}$ was prepared according to the general procedure for starting material synthesis on a $5 \mathrm{mmol}$ scale and was obtained after silica column chromatography ( $n$-pentane : $\mathrm{Et}_{3} \mathrm{~N}$ 1:1 $\rightarrow$ 20:1); 69\%, (934 mg); orange solid.

${ }^{1}$ H NMR (600 MHz, Chloroform- $d$ ): $\delta=7.99-7.94(\mathrm{~m}, 1 \mathrm{H}), 7.95-7.84(\mathrm{~m}, 1 \mathrm{H}), 7.55-7.43$ (m, 1H), $7.44-7.35(\mathrm{~m}, 1 \mathrm{H}), 7.33-7.27(\mathrm{~m}, 2 \mathrm{H}), 7.08-6.95(\mathrm{~m}, 2 \mathrm{H}), 3.85(\mathrm{~s}, 3 \mathrm{H}) \mathrm{ppm}$.

${ }^{13}$ C NMR (151 MHz, Chloroform- $d$ ): $\delta=159.1,149.0,133.9,129.8,128.5,128.4,118.9,118.8$, $117.6,115.2,55.4 \mathrm{ppm}$.

IR (KBr): 3871, 3384, 3076, 2999, 2962, 2933, 2828, 2325, 2166, 2125, 2037, 1899, 1786, $1655,1605,1566,1521,1346,1244,1177,1110,1084,1031,991,961,904,866,833,794$, $727,669 \mathrm{~cm}^{-1}$.

Note: HRMS analysis of compound $\mathbf{1 h}$ was not possible due to the low stability. 


\section{2-(Diazo(4-nitrophenyl)methyl)-1,4-dimethylbenzene (1j)}<smiles>Cc1ccc(C)c(C(=N)c2ccc([N+](=O)[O-])cc2)c1</smiles>

Compound $\mathbf{1} \mathbf{j}$ was prepared according to the general procedure for starting material synthesis on a $5 \mathrm{mmol}$ scale and was obtained after silica column chromatography ( $n$-pentane : $\mathrm{Et}_{3} \mathrm{~N}$ 1:1 $\rightarrow$ 20:1); 44\%, (585 mg); orange oil.

${ }^{1}$ H NMR (400 MHz, Chloroform- $d$ ): $\delta=8.27-8.06(\mathrm{~m}, 2 \mathrm{H}), 7.24(\mathrm{t}, J=3.9 \mathrm{~Hz}, 1 \mathrm{H}), 7.22-$ $7.14(\mathrm{~m}, 2 \mathrm{H}), 6.99-6.90(\mathrm{~m}, 2 \mathrm{H}), 2.34(\mathrm{~s}, 3 \mathrm{H}), 2.21(\mathrm{~s}, 3 \mathrm{H}) \mathrm{ppm}$.

${ }^{13}$ C NMR (101 MHz, Chloroform- $d$ ): $\delta=143.6,140.2,136.7,135.5,131.6,131.2,130.5,125.0$, $124.5,123.6,121.4,20.8,19.3 \mathrm{ppm}$.

IR (KBr): 3623, 3357, 3077, 2917, 2863, 2733, 2644, 2323, 2120, 2039, 1788, 1721, 1662 , 1585, 1492, 1378, 1305, 1257, 1189, 1110, 1039, 1006, 953, 903, 839, 803, 744, $688 \mathrm{~cm}^{-1}$.

Note: HRMS analysis of compound $\mathbf{1} \mathbf{j}$ was not possible due to the low stability.

\section{$N$-Benzhydrylaniline (10a)}<smiles>c1ccc(NC(c2ccccc2)c2ccccc2)cc1</smiles>

The titled compound 10a was synthesized according to the general procedure GP- 1 on a 0.2 mmol scale and was obtained after silica gel column chromatography using $n$-hexane/EtOAc $80: 1 \rightarrow 40: 1$ as colorless oil $(86 \%, 45 \mathrm{mg})$.

${ }^{1}$ H NMR (600 MHz, Chloroform- $d$ ): $\delta=7.40-7.31(\mathrm{~m}, 8 \mathrm{H}), 7.29-7.23(\mathrm{~m}, 2 \mathrm{H}), 7.16-7.06$ $(\mathrm{m}, 2 \mathrm{H}), 6.73-6.67(\mathrm{~m}, 1 \mathrm{H}), 6.58-6.50(\mathrm{~m}, 2 \mathrm{H}), 5.51(\mathrm{~s}, 1 \mathrm{H}), 4.24(\mathrm{~s}, 1 \mathrm{H}) \mathrm{ppm}$.

${ }^{13}$ C NMR (151 MHz, Chloroform- $d$ ): $\delta=147.3,142.9,129.1,128.7,127.4,127.3,117.6,113.4$, $63.0 \mathrm{ppm}$.

HRMS (ESI): $m / z$ : $[\mathrm{M}+\mathrm{Na}]^{+}$Calcd. for $\mathrm{C}_{19} \mathrm{H}_{17} \mathrm{NNa}^{+}: 282.1253$; Found: 282.1251 .

IR (KBr): 3410, 3054, 3026, 2924, 2852, 2324, 2159, 2086, 1951, 1813, 1765, 1599, 1497 , $1450,1425,1312,1266,1180,1105,1072,1027,992,919,872,822,744,695 \mathrm{~cm}^{-1}$. 


\section{$N$-Benzhydryl-4-methylaniline (10b)}<smiles>Cc1ccc(NC(c2ccccc2)c2ccccc2)cc1</smiles>

The titled compound 10b was synthesized according to the general procedure GP-1 on a 0.2 mmol scale and was obtained after silica gel column chromatography using $n$-hexane/EtOAc $40: 1 \rightarrow 20: 1$ as colorless oil $(66 \%, 36 \mathrm{mg})$.

${ }^{1}$ H NMR (600 MHz, Chloroform- $d$ ): $\delta=7.42-7.33(\mathrm{~m}, 8 \mathrm{H}), 7.31-7.27(\mathrm{~m}, 2 \mathrm{H}), 6.97(\mathrm{~d}, J=$ $8.0 \mathrm{~Hz}, 2 \mathrm{H}), 6.54-6.47(\mathrm{~m}, 2 \mathrm{H}), 5.50(\mathrm{~s}, 1 \mathrm{H}), 4.15(\mathrm{~s}, 1 \mathrm{H}), 2.25(\mathrm{~s}, 3 \mathrm{H}) \mathrm{ppm}$.

${ }^{13}$ C NMR (151 MHz, Chloroform- $d$ ): $\delta=145.1,143.1,129.6,128.7,127.4,127.3,126.8,113.5$, 63.3, $20.4 \mathrm{ppm}$.

HRMS (ESI): $m / z$ : $[\mathrm{M}+\mathrm{Na}]^{+}$Calcd. for $\mathrm{C}_{20} \mathrm{H}_{19} \mathrm{NNa}^{+}$: 296.1409; Found: 296.1405.

IR (KBr): 3409, 3026, 2919, 2862, 2733, 2160, 2045, 1952,1876, 1810, 1736, 1614, 1514, $1450,1401,1301,1264,1239,1182,1122,1065,1029,911,806,774,740,697 \mathrm{~cm}^{-1}$.

\section{$N$-Benzhydryl-4-methoxyaniline (10c)}<smiles>COc1ccc(NC(c2ccccc2)c2ccccc2)cc1</smiles>

The titled compound 10c was synthesized according to the general procedure GP-1 on a 0.2 mmol scale and was obtained after silica gel column chromatography using $n$-hexane/EtOAc $20: 1 \rightarrow 10: 1$ as colorless oil $(81 \%, 47 \mathrm{mg})$.

${ }^{1}$ H NMR (600 MHz, Chloroform- $d$ ): $\delta=7.40(\mathrm{~d}, J=8.2 \mathrm{~Hz}, 4 \mathrm{H}), 7.37-7.33(\mathrm{~m}, 4 \mathrm{H}), 7.30$ $7.24(\mathrm{~m}, 2 \mathrm{H}), 6.77-6.70(\mathrm{~m}, 2 \mathrm{H}), 6.55-6.47(\mathrm{~m}, 2 \mathrm{H}), 5.45(\mathrm{~s}, 1 \mathrm{H}), 4.03(\mathrm{~s}, 1 \mathrm{H}), 3.74(\mathrm{~s}, 3 \mathrm{H})$ ppm.

${ }^{13}$ C NMR (151 MHz, Chloroform- $d$ ): $\delta=152.1,143.2,141.7,128.7,127.4,127.3,114.7,114.6$, $63.8,55.7 \mathrm{ppm}$.

HRMS (ESI): $m / z$ : $[\mathrm{M}+\mathrm{Na}]^{+}$Calcd. for $\mathrm{C}_{20} \mathrm{H}_{19} \mathrm{NNaO}^{+}: 321.1358$; Found: 312.1354 .

IR (KBr): 3400, 3059, 3027, 2937, 2832, 2246, 2180, 2061, 1951, 1813, 1736, 1659, 1598, $1507,1451,1404,1297,1236,1177,1117,1092,1033,962,910,818,770,740,698 \mathrm{~cm}^{-1}$. 


\section{$N$-Benzhydryl-4-fluoroaniline (10d)}<smiles>Fc1ccc(NC(c2ccccc2)c2ccccc2)cc1</smiles>

The titled compound 10d was synthesized according to the general procedure GP-1 on a 0.2 mmol scale and was obtained after silica gel column chromatography using $n$-hexane/EtOAc $40: 1 \rightarrow 20: 1$ as colorless oil $(86 \%, 47 \mathrm{mg})$.

${ }^{1}$ H NMR (600 MHz, Chloroform- $d$ ): $\delta=7.42-7.33(\mathrm{~m}, 8 \mathrm{H}), 7.33-7.24(\mathrm{~m}, 2 \mathrm{H}), 6.89-6.80$ $(\mathrm{m}, 2 \mathrm{H}), 6.53-6.44(\mathrm{~m}, 2 \mathrm{H}), 5.47(\mathrm{~s}, 1 \mathrm{H}), 4.17(\mathrm{~s}, 1 \mathrm{H}) \mathrm{ppm}$.

${ }^{13}$ C NMR (151 MHz, Chloroform- $d$ ): $\delta=155.9(\mathrm{~d}, J=235.5 \mathrm{~Hz}), 143.7(\mathrm{~d}, J=2.1 \mathrm{~Hz}), 142.8$, 128.8, 127.46, 127.41, $115.5(\mathrm{~d}, J=22.3 \mathrm{~Hz}), 114.2(\mathrm{~d}, J=7.3 \mathrm{~Hz}), 63.6 \mathrm{ppm}$

${ }^{19}$ F NMR (564 MHz, Chloroform- $d$ ) $\delta=-127.7$ ppm.

HRMS (ESI): $m / z$ : [M] $]^{+}$Calcd. for $\mathrm{C}_{19} \mathrm{H}_{16} \mathrm{FN}^{+}: 277.1261$; Found: 277.1257.

IR (KBr): 3413, 3060, 3029, 2853, 2155, 1952, 1849, 1732, 1604, 1505, 1451, 1398, 1309 , $1265,1216,1157,1110,1065,1028,911,818,780,741,698 \mathrm{~cm}^{-1}$.

\section{$N$-Benzhydryl-4-chloroaniline (10e)}<smiles>Clc1ccc(NC(c2ccccc2)c2ccccc2)cc1</smiles>

The titled compound 10e was synthesized according to the general procedure GP-1 on a 0.2 mmol scale and was obtained after silica gel column chromatography using $n$-hexane/EtOAc $40: 1 \rightarrow 20: 1$ as colorless oil $(90 \%, 53 \mathrm{mg})$.

${ }^{1} \mathbf{H}$ NMR $(600 \mathrm{MHz}$, Chloroform- $d$ ): $\delta=7.40-7.34(\mathrm{~m}, 8 \mathrm{H}), 7.33-7.28(\mathrm{~m}, 2 \mathrm{H}), 7.11-7.07$ $(\mathrm{m}, 2 \mathrm{H}), 6.52-6.47(\mathrm{~m}, 2 \mathrm{H}), 5.50(\mathrm{~d}, J=3.2 \mathrm{~Hz}, 1 \mathrm{H}), 4.28(\mathrm{~d}, J=3.7 \mathrm{~Hz}, 1 \mathrm{H}) \mathrm{ppm}$.

${ }^{13}$ C NMR (151 MHz, Chloroform- $d$ ): $\delta=145.8,142.4,128.9,128.8,127.5$, 127.4, 122.3, 114.6, $63.1 \mathrm{ppm}$.

HRMS (ESI): $m / z$ : [M] ${ }^{+}$Calcd. for $\mathrm{C}_{19} \mathrm{H}_{16} \mathrm{ClN}^{+}$: 293.0965; Found: 293.0957 .

IR (KBr): 3414, 3060, 3028, 2854, 2579, 2248, 2181, 2048, 1952, 1867, 1812, 1736, 1596, 1493, 1451, 1396, 1311, 1266, 1237, 1178, 1088, 1028, 1004, 908, 813, 739, $697 \mathrm{~cm}^{-1}$. 


\section{$N$-Benzhydryl-4-bromoaniline (10f)}<smiles>Brc1ccc(NC(c2ccccc2)c2ccccc2)cc1</smiles>

The titled compound $\mathbf{1 0 f}$ was synthesized according to the general procedure GP- 1 on a 0.2 mmol scale and was obtained after silica gel column chromatography using $n$-hexane/EtOAc $40: 1 \rightarrow 20: 1$ as colorless oil $(80 \%, 54 \mathrm{mg})$.

${ }^{1}$ H NMR $(600 \mathrm{MHz}$, Chloroform- $d$ ): $\delta=7.35-7.31(\mathrm{~m}, 8 \mathrm{H}), 7.29-7.24(\mathrm{~m}, 2 \mathrm{H}), 7.20-7.15$ $(\mathrm{m}, 2 \mathrm{H}), 6.44-6.37(\mathrm{~m}, 2 \mathrm{H}), 5.45(\mathrm{~d}, J=3.7 \mathrm{~Hz}, 1 \mathrm{H}), 4.26(\mathrm{~d}, J=3.9 \mathrm{~Hz}, 1 \mathrm{H}) \mathrm{ppm}$.

${ }^{13}$ C NMR (151 MHz, Chloroform- $d$ ): $\delta=146.2,142.3,131.8,128.8,127.5,127.3,115.0,109.3$, $62.9 \mathrm{ppm}$.

HRMS (ESI): $m / z$ : [M] ${ }^{+}$Calcd. for $\mathrm{C}_{19} \mathrm{H}_{16} \mathrm{BrN}^{+}$: 337.0460; Found: 337.0449 .

IR (KBr): 3408, 3029, 2923, 2856, 2323, 2093, 1863, 1819, 1733, 1587, 1488, 1450, 1390 , $1341,1307,1268,1234,1178,1119,1067,1027,997,959,917,810,747,696,663 \mathrm{~cm}^{-1}$.

\section{4-(Benzhydrylamino)benzonitrile (10g)}<smiles>N#Cc1ccc(NC(c2ccccc2)c2ccccc2)cc1</smiles>

The titled compound $10 \mathrm{~g}$ was synthesized according to the general procedure GP- 1 on a 0.2 mmol scale and was obtained after silica gel column chromatography using $n$-hexane/EtOAc $20: 1 \rightarrow 10: 1$ as colorless oil $60 \%, 34 \mathrm{mg})$.

${ }^{1}$ H NMR (600 MHz, Chloroform- $d$ ): $\delta=7.39-7.33(\mathrm{~m}, 6 \mathrm{H}), 7.32-7.27(\mathrm{~m}, 6 \mathrm{H}), 6.54-6.50$ $(\mathrm{m}, 2 \mathrm{H}), 5.56(\mathrm{~d}, J=4.6 \mathrm{~Hz}, 1 \mathrm{H}), 4.74(\mathrm{~d}, J=4.7 \mathrm{~Hz}, 1 \mathrm{H}) \mathrm{ppm}$.

${ }^{13}$ C NMR (151 MHz, Chloroform- $d$ ): $\delta=150.1,141.3,133.6,128.9,127.8,127.3,120.2,113.1$, $99.5,62.3 \mathrm{ppm}$.

HRMS (ESI): $m / z$ : $[\mathrm{M}+\mathrm{Na}]^{+}$Calcd. for $\mathrm{C}_{20} \mathrm{H}_{16} \mathrm{~N}_{2} \mathrm{Na}^{+}: 307.1205$; Found: 307.1209 .

IR (KBr): 3820, 3357, 3060, 3030, 2860, 2604, 2318, 2213, 1952, 1892, 1760, 1602, 1516, $1451,1417,1333,1274,1173,1129,1093,1065,1028,909,823,731,699 \mathrm{~cm}^{-1}$. 


\section{$N$-Benzhydryl-3-methoxyaniline (10h)}<smiles>COc1cccc(NC(c2ccccc2)c2ccccc2)c1</smiles>

The titled compound 10h was synthesized according to the general procedure GP- 1 on a 0.2 mmol scale and was obtained after silica gel column chromatography using $n$-hexane/EtOAc $20: 1 \rightarrow 10: 1$ as colorless oil ( $83 \%, 48 \mathrm{mg})$.

${ }^{1}$ H NMR (600 MHz, Chloroform- $d$ ): $\delta=7.40-7.32(\mathrm{~m}, 8 \mathrm{H}), 7.30-7.25(\mathrm{~m}, 2 \mathrm{H}), 7.05(\mathrm{t}, J=$ $8.1 \mathrm{~Hz}, 1 \mathrm{H}), 6.32-6.26(\mathrm{~m}, 1 \mathrm{H}), 6.23-6.18(\mathrm{~m}, 1 \mathrm{H}), 6.15-6.10(\mathrm{~m}, 1 \mathrm{H}), 5.53(\mathrm{~s}, 1 \mathrm{H}), 4.28$ (s, 1H), $3.71(\mathrm{~s}, 3 \mathrm{H}) \mathrm{ppm}$.

${ }^{13}$ C NMR (151 MHz, Chloroform- $d$ ): $\delta=160.6,148.7,142.8,129.8,128.7,127.4,127.3,106.5$, 102.9, 99.5, 63.0, $55.0 \mathrm{ppm}$.

HRMS (ESI): $m / z$ : [M + Na] $]^{+}$Calcd. $\mathrm{C}_{20} \mathrm{H}_{19} \mathrm{NNaO}^{+}: 312.1358$; Found: 312.1353 .

IR (KBr): 3407, 3059, 3027, 2936, 2835, 2335, 2246, 2084, 1894, 1810, 1600, 1494, 1451, $1338,1304,1278,1245,1208,1160,1106,1040,991,970,944,909,828,739,696 \mathrm{~cm}^{-1}$.

\section{$N$-Benzhydryl-3-bromoaniline (10i)}<smiles>Brc1cccc(NC(c2ccccc2)c2ccccc2)c1</smiles>

The titled compound $\mathbf{1 0} \mathbf{i}$ was synthesized according to the general procedure GP-1 on a 0.2 mmol scale and was obtained after silica gel column chromatography using $n$-hexane/EtOAc $40: 1 \rightarrow 20: 1$ as colorless oil $(93 \%, 63 \mathrm{mg})$.

${ }^{1}$ H NMR (600 MHz, Chloroform- $d$ ): $\delta=7.38-7.33(\mathrm{~m}, 8 \mathrm{H}), 7.32-7.27(\mathrm{~m}, 2 \mathrm{H}), 6.97(\mathrm{t}, J=$ $8.0 \mathrm{~Hz}, 1 \mathrm{H}), 6.84-6.80(\mathrm{~m}, 1 \mathrm{H}), 6.74-6.71(\mathrm{~m}, 1 \mathrm{H}), 6.49-6.44(\mathrm{~m}, 1 \mathrm{H}), 5.51(\mathrm{~d}, J=4.2 \mathrm{~Hz}$, $1 \mathrm{H}), 4.32(\mathrm{~d}, J=4.3 \mathrm{~Hz}, 1 \mathrm{H}) \mathrm{ppm}$.

${ }^{13}$ C NMR (151 MHz, Chloroform- $d$ ): $\delta=148.5,142.2,130.4,128.8,127.5,127.3,123.0,120.5$, $116.2,111.9,62.7 \mathrm{ppm}$.

HRMS (ESI): $m / z$ : $[\mathrm{M}+\mathrm{Na}]^{+}$Calcd. for $\mathrm{C}_{19} \mathrm{H}_{16} \mathrm{BrNNa}^{+}: 360.0358$; Found: 360.0351 .

IR (KBr): 3411, 3060, 3027, 2856, 2336, 2089, 2018, 1953, 1890, 1810, 1761, 1656, 1591, 1486, 1451, 1410, 1317, 1278, 1234, 1172, 1108, 1065, 1027, 985, 906, 843, 739, $697 \mathrm{~cm}^{-1}$. 


\section{$N$-Benzhydryl-3-iodoaniline (10j)}<smiles>Ic1cccc(NC(c2ccccc2)c2ccccc2)c1</smiles>

The titled compound $\mathbf{1 0 j}$ was synthesized according to the general procedure GP- 1 on a 0.2 mmol scale and was obtained after silica gel column chromatography using $n$-hexane/EtOAc $40: 1 \rightarrow 20: 1$ as colorless oil $(83 \%, 64 \mathrm{mg})$.

${ }^{1}$ H NMR (600 MHz, Chloroform- $d$ ): $\delta=7.42-7.32(\mathrm{~m}, 8 \mathrm{H}), 7.34-7.27(\mathrm{~m}, 2 \mathrm{H}), 7.06-7.01$ $(\mathrm{m}, 1 \mathrm{H}), 6.96(\mathrm{t}, J=1.9 \mathrm{~Hz}, 1 \mathrm{H}), 6.84(\mathrm{t}, J=7.9 \mathrm{~Hz}, 1 \mathrm{H}), 6.51-6.47(\mathrm{~m}, 1 \mathrm{H}), 5.50(\mathrm{~d}, J=3.9$ $\mathrm{Hz}, 1 \mathrm{H}), 4.28(\mathrm{~d}, J=4.2 \mathrm{~Hz}, 1 \mathrm{H}) \mathrm{ppm}$.

${ }^{13}$ C NMR (151 MHz, Chloroform- $d$ ): $\delta=148.4,142.3,130.6,128.8,127.5,127.4,126.6,122.3$, 112.4, 95.0, $62.7 \mathrm{ppm}$.

HRMS (APCI): $m / z:[\mathrm{M}+\mathrm{H}]^{+}$Calcd. for $\mathrm{C}_{19} \mathrm{H}_{17} \mathrm{IN}^{+}:$386.0400; Found: 386.0413 .

IR (KBr): 3408, 3059, 3027, 2856, 2337, 2163, 1953, 1890, 1812, 1706, 1656, 1585, 1485, $1451,1406,1315,1281,1233,1173,1059,1027,983,906,842,736,696 \mathrm{~cm}^{-1}$.

\section{$N$-Benzhydryl-2-chloroaniline (10k)}<smiles>Clc1ccccc1NC(c1ccccc1)c1ccccc1</smiles>

The titled compound 10k was synthesized according to the general procedure GP-1 on a 0.2 mmol scale and was obtained after silica gel column chromatography using $n$-hexane/EtOAc $40: 1 \rightarrow 20: 1$ as colorless oil $(72 \%, 42 \mathrm{mg})$.

${ }^{1}$ H NMR (600 MHz, Chloroform- $d$ ): $\delta=7.41-7.34(\mathrm{~m}, 8 \mathrm{H}), 7.32-7.26(\mathrm{~m}, 3 \mathrm{H}), 7.02(\mathrm{t}, J=$ $7.8 \mathrm{~Hz}, 1 \mathrm{H}), 6.67-6.61(\mathrm{~m}, 1 \mathrm{H}), 6.52-6.47(\mathrm{~m}, 1 \mathrm{H}), 5.58(\mathrm{~d}, J=4.5 \mathrm{~Hz}, 1 \mathrm{H}), 4.94(\mathrm{~d}, J=4.6$ $\mathrm{Hz}, 1 \mathrm{H}) \mathrm{ppm}$.

${ }^{13}$ C NMR (151 MHz, Chloroform- $d$ ): $\delta=143.0,142.3,129.0,128.8,127.6,127.5,127.3,119.3$, 117.6, 112.6, $62.6 \mathrm{ppm}$.

HRMS (ESI): $m / z$ : [M] $]^{+}$Calcd. for $\mathrm{C}_{19} \mathrm{H}_{16} \mathrm{ClN}^{+}$: 293.0965; Found: 293.0956 .

IR (KBr): 3413, 3061, 3028, 2923, 2333, 2156, 1959, 1894, 1818, 1767, 1660, 1592, 1497 , 1446, 1318, 1281, 1239, 1184, 1130, 1069, 1027, 922, 883, 829, 743, $696 \mathrm{~cm}^{-1}$. 


\section{$N$-Benzhydryl-2-bromoaniline (101)}<smiles>Brc1ccccc1NC(c1ccccc1)c1ccccc1</smiles>

The titled compound $\mathbf{1 0 1}$ was synthesized according to the general procedure GP-1 on a 0.2 mmol scale and was obtained after silica gel column chromatography using $n$-hexane/EtOAc $40: 1 \rightarrow 20: 1$ as colorless oil ( $59 \%, 40 \mathrm{mg})$.

${ }^{1}$ H NMR (600 MHz, Chloroform- $d$ ): $\delta=7.39-7.33(\mathrm{~m}, 1 \mathrm{H}), 7.31-7.23(\mathrm{~m}, 8 \mathrm{H}), 7.21-7.16$ (m, 2H), $6.99-6.87(\mathrm{~m}, 1 \mathrm{H}), 6.51-6.45(\mathrm{~m}, 1 \mathrm{H}), 6.41-6.34(\mathrm{~m}, 1 \mathrm{H}), 5.48(\mathrm{~d}, J=4.6 \mathrm{~Hz}$, $1 \mathrm{H}), 4.86(\mathrm{~d}, J=4.6 \mathrm{~Hz}, 1 \mathrm{H}) \mathrm{ppm}$.

${ }^{13}$ C NMR (151 MHz, Chloroform- $d$ ): $\delta=144.0,142.3,132.2,128.8,128.3,127.5,127.3,118.1$, 112.7, 109.9, $62.7 \mathrm{ppm}$.

HRMS (ESI): $m / z$ : [M] ${ }^{+}$Calcd. for $\mathrm{C}_{19} \mathrm{H}_{16} \mathrm{BrN}^{+}:$337.0460; Found: 337.0447.

IR (KBr): 3408, 3061, 3028, 2924, 2853, 2630, 2326, 2177, 2036, 1954, 1888, 1808, 1709 , 1591, 1496, 1451, 1423, 1315, 1235, 1179, 1126, 1065, 1021, 917, 885, 833, 739, $697 \mathrm{~cm}^{-1}$.

\section{$N$-Benzhydryl-2-methylaniline (10m)}<smiles>Cc1ccccc1NC(c1ccccc1)c1ccccc1</smiles>

The titled compound $\mathbf{1 0 m}$ was synthesized according to the general procedure GP-1 on a 0.2 mmol scale and was obtained after silica gel column chromatography using $n$-hexane/EtOAc $80: 1 \rightarrow 40: 1$ as colorless oil $(64 \%, 35 \mathrm{mg})$.

${ }^{1}$ H NMR (600 MHz, Chloroform- $d$ ): $\delta=7.39-7.36(\mathrm{~m}, 4 \mathrm{H}), 7.36-7.32(\mathrm{~m}, 4 \mathrm{H}), 7.29-7.24$ (m, 2H), $7.12-7.06(\mathrm{~m}, 1 \mathrm{H}), 7.02-6.94(\mathrm{~m}, 1 \mathrm{H}), 6.69-6.63(\mathrm{~m}, 1 \mathrm{H}), 6.43(\mathrm{~d}, J=8.1 \mathrm{~Hz}$, $1 \mathrm{H}), 5.57(\mathrm{~s}, 1 \mathrm{H}), 4.07(\mathrm{~s}, 1 \mathrm{H}), 2.20(\mathrm{~s}, 3 \mathrm{H}) \mathrm{ppm}$.

${ }^{13}$ C NMR (151 MHz, Chloroform- $d$ ): $\delta=145.2,143.0,129.9,128.7,127.4,127.3,126.9,122.0$, $117.3,111.3,62.8,17.6 \mathrm{ppm}$.

HRMS (ESI): $m / z$ : [M + Na] $]^{+}$Calcd. for $\mathrm{C}_{20} \mathrm{H}_{19} \mathrm{NNaO}^{+}: 296.1409$; Found: 296.1406 .

IR (KBr): 3432, 3058, 3027, 2920, 2854, 2324, 2179, 2089, 1950, 1886, 1807, 1759, 1668 , $1601,1501,1446,1379,1309,1264,1179,1127,1053,1028,985,884,837,743,698 \mathrm{~cm}^{-1}$. 


\section{$N$-Benzhydryl-2-methoxyaniline (10n)}<smiles>COc1ccccc1NC(c1ccccc1)c1ccccc1</smiles>

The titled compound 10n was synthesized according to the general procedure GP-1 on a 0.2 mmol scale and was obtained after silica gel column chromatography using $n$-hexane/EtOAc $40: 1 \rightarrow 20: 1$ as colorless oil $(67 \%, 39 \mathrm{mg})$.

${ }^{1}$ H NMR (600 MHz, Chloroform- $d$ ): $\delta=7.29(\mathrm{~d}, J=8.3 \mathrm{~Hz}, 4 \mathrm{H}), 7.27-7.21(\mathrm{~m}, 4 \mathrm{H}), 7.20-$ $7.14(\mathrm{~m}, 2 \mathrm{H}), 6.73-6.68(\mathrm{~m}, 1 \mathrm{H}), 6.67-6.62(\mathrm{~m}, 1 \mathrm{H}), 6.61-6.54(\mathrm{~m}, 1 \mathrm{H}), 6.33-6.29(\mathrm{~m}$, $1 \mathrm{H}), 5.41(\mathrm{~d}, J=3.2 \mathrm{~Hz}, 1 \mathrm{H}), 4.76(\mathrm{~s}, 1 \mathrm{H}), 3.76(\mathrm{~s}, 3 \mathrm{H}) \mathrm{ppm}$.

${ }^{13}$ C NMR (151 MHz, Chloroform- $d$ ): $\delta=146.8,143.1,137.3,128.7,127.4,127.2,121.1,116.7$, 111.2, 109.2, 62.8, $55.4 \mathrm{ppm}$.

HRMS (ESI): $m / z$ : $[\mathrm{M}+\mathrm{Na}]^{+}$Calcd. for $\mathrm{C}_{20} \mathrm{H}_{19} \mathrm{NNaO}^{+}: 312.1358$; Found: 312.1354 .

IR (KBr): 3409, 3057, 2926, 2852, 2319, 2179, 2107, 1952, 1892, 1811, 1736, 1599, 1508, 1451, 1420, 1347, 1302, 1263, 1230, 1177, 1122, 1089, 1051, 1024, 921, 897, 834, 777, 731, $695 \mathrm{~cm}^{-1}$.

\section{Methyl 2-(benzhydrylamino)benzoate (10o)}<smiles>CC(=O)c1ccccc1NC(c1ccccc1)c1ccccc1</smiles>

The titled compound $\mathbf{1 0 0}$ was synthesized according to the general procedure GP- 1 on a 0.2 mmol scale and was obtained after silica gel column chromatography using $n$-hexane/EtOAc $20: 1 \rightarrow 10: 1$ as colorless oil $(65 \%, 41 \mathrm{mg})$.

${ }^{1}$ H NMR (600 MHz, Chloroform- $d$ ): $\delta=8.46(\mathrm{~d}, J=5.5 \mathrm{~Hz}, 1 \mathrm{H}), 8.00-7.82(\mathrm{~m}, 1 \mathrm{H}), 7.39-$ $7.35(\mathrm{~m}, 4 \mathrm{H}), 7.34-7.30(\mathrm{~m}, 4 \mathrm{H}), 7.27-7.20(\mathrm{~m}, 3 \mathrm{H}), 6.61-6.57(\mathrm{~m}, 1 \mathrm{H}), 6.54(\mathrm{~d}, J=8.5$ $\mathrm{Hz}, 1 \mathrm{H}), 5.64(\mathrm{~d}, J=5.5 \mathrm{~Hz}, 1 \mathrm{H}), 3.85(\mathrm{~s}, 3 \mathrm{H}) \mathrm{ppm}$.

${ }^{13}$ C NMR (151 MHz, Chloroform- $d$ ): $\delta=169.1,149.9,142.5,134.4,131.5,128.8,127.3,127.2$, $115.1,112.7,110.4,61.8,51.5 \mathrm{ppm}$.

HRMS (ESI): $m / z$ : [M] $]^{+}$Calcd. for $\mathrm{C}_{21} \mathrm{H}_{19} \mathrm{NO}_{2}{ }^{+}: 317.1410$; Found: 317.1401 .

IR (KBr): 3356, 3066, 3026, 2923, 2854, 2652, 2341, 2209, 2159, 2022, 1890, 1813, 1729 , $1677,1577,1503,1445,1320,1259,1234,1163,1105,1077,1047,1028,940,918,881,843$, $804,742,695 \mathrm{~cm}^{-1}$. 


\section{$N$-Benzhydrylnaphthalen-1-amine (10p)}<smiles>c1ccc(C(Nc2cccc3ccccc23)c2ccccc2)cc1</smiles>

The titled compound 10p was synthesized according to the general procedure GP-1 on a 0.2 mmol scale and was obtained after silica gel column chromatography using $n$-hexane/EtOAc $40: 1 \rightarrow 20: 1$ as colorless oil $(68 \%, 42 \mathrm{mg})$.

${ }^{1}$ H NMR $(600 \mathrm{MHz}$, Chloroform- $d$ ): $\delta=7.81-7.77(\mathrm{~m}, 1 \mathrm{H}), 7.74-7.68(\mathrm{~m}, 1 \mathrm{H}), 7.42-7.30$ $(\mathrm{m}, 6 \mathrm{H}), 7.29-7.24(\mathrm{~m}, 4 \mathrm{H}), 7.22-7.17(\mathrm{~m}, 2 \mathrm{H}), 7.16-7.11(\mathrm{~m}, 2 \mathrm{H}), 6.38-6.32(\mathrm{~m}, 1 \mathrm{H})$, $5.61(\mathrm{~s}, 1 \mathrm{H}), 4.83(\mathrm{~s}, 1 \mathrm{H}) \mathrm{ppm}$.

${ }^{13}$ C NMR (151 MHz, Chloroform- $d$ ): $\delta=142.7,142.2,134.2,128.8,128.7,127.5,127.4,126.5$, $125.7,124.8,123.4,119.9,117.7,106.4,63.1 \mathrm{ppm}$.

HRMS (ESI): $m / z$ : $[\mathrm{M}+\mathrm{K}]^{+}$Calcd. $\mathrm{C}_{23} \mathrm{H}_{19} \mathrm{KN}^{+}: 348.1149$; Found: 348.1139.

IR (KBr): 3430, 3207, 3059, 2926, 2854, 2246, 2161, 2028, 1896, 1813, 1730, 1667, 1624, $1579,1522,1472,1405,1343,1279,1251,1111,1071,1031,981,845,766,733,697 \mathrm{~cm}^{-1}$.

\section{$N$-Benzhydrylbenzo $[d][1,3]$ dioxol-5-amine (10q)}<smiles>c1ccc(C(Nc2ccc3c(c2)OCO3)c2ccccc2)cc1</smiles>

The titled compound 10q was synthesized according to the general procedure GP-1 on a 0.2 mmol scale and was obtained after silica gel column chromatography using $n$-hexane/EtOAc 20:1 $\rightarrow 10: 1$ as colorless oil $(56 \%, 34 \mathrm{mg})$.

${ }^{1}$ H NMR (600 MHz, Chloroform- $d$ ): $\delta=7.38-7.30(\mathrm{~m}, 8 \mathrm{H}), 7.29-7.23(\mathrm{~m}, 2 \mathrm{H}), 6.61-6.58$ (m, 1H), $6.19(\mathrm{~d}, J=2.3 \mathrm{~Hz}, 1 \mathrm{H}), 5.99-5.95(\mathrm{~m}, 1 \mathrm{H}), 5.82(\mathrm{~s}, 2 \mathrm{H}), 5.41(\mathrm{~s}, 1 \mathrm{H}), 4.05(\mathrm{~s}, 1 \mathrm{H})$. ppm.

${ }^{13}$ C NMR (151 MHz, Chloroform- $d$ ): $\delta=148.1,143.1,142.9,139.6,128.7,127.38,127.36$, 108.5, 105.1, 100.5, 96.4, $63.8 \mathrm{ppm}$.

HRMS (ESI): $m / z$ : $[\mathrm{M}+\mathrm{H}]^{+}$Calcd. for $\mathrm{C}_{20} \mathrm{H}_{18} \mathrm{NO}_{2}{ }^{+}$: 304.1332; Found: 304.1341 .

IR (KBr): 3407, 3060, 3027, 2880, 2774, 2247, 2045, 1953, 1811, 1756, 1631, 1487, 1450, 1343, 1293, 1200, 1138, 1096, 1035, 929, 910, 815, 788, 734, $698 \mathrm{~cm}^{-1}$. 


\section{$N$-(Bis(4-methoxyphenyl)methyl)aniline (10r)}<smiles>COc1ccc(C(Nc2ccccc2)c2ccc(OC)cc2)cc1</smiles>

The titled compound 10r was synthesized according to the general procedure GP-1 on a 0.2 mmol scale and was obtained after silica gel column chromatography using $n$-hexane/EtOAc $20: 1 \rightarrow 10: 1$ as colorless oil $(42 \%, 66 \mathrm{mg})$.

${ }^{1}$ H NMR $(600 \mathrm{MHz}$, Chloroform- $d$ ): $\delta=7.32-7.25(\mathrm{~m}, 4 \mathrm{H}), 7.18-7.12(\mathrm{~m}, 2 \mathrm{H}), 6.92-6.88$ (m, 4H), $6.74-6.70(\mathrm{~m}, 1 \mathrm{H}), 6.60-6.55(\mathrm{~m}, 2 \mathrm{H}), 5.46(\mathrm{~s}, 1 \mathrm{H}), 4.20(\mathrm{~s}, 1 \mathrm{H}), 3.82(\mathrm{~s}, 6 \mathrm{H}) \mathrm{ppm}$. ${ }^{13}$ C NMR (151 MHz, Chloroform-d): $\delta=158.7,147.4,135.4,129.1,128.5,117.5,114.0,113.4$, 61.7, $55.2 \mathrm{ppm}$.

HRMS (ESI): $m / z$ : $[\mathrm{M}+\mathrm{Na}]^{+}$Calcd. $\mathrm{C}_{21} \mathrm{H}_{21} \mathrm{NNaO}_{2}{ }^{+}: 342.1464$; Found: 342.1452 .

IR (KBr): 3402, 3003, 2954, 2835, 2331, 2070, 1891, 1769, 1602, 1502, 1461, 1427, 1305 , $1242,1173,1094,1064,1031,907,816,733,692 \mathrm{~cm}^{-1}$.

\section{$N$-((4-Chlorophenyl)(4-methoxyphenyl)methyl)aniline (10s)}<smiles>COc1ccc(C(Nc2ccccc2)c2ccc(Cl)cc2)cc1</smiles>

The titled compound 10s was synthesized according to the general procedure GP-1 on a 0.2 mmol scale and was obtained after silica gel column chromatography using $n$-hexane/EtOAc 40:1 $\rightarrow 20: 1$ as colorless solid ( $73 \%, 47 \mathrm{mg})$.

${ }^{1}$ H NMR (600 MHz, Chloroform- $d$ ): $\delta=7.26-7.18(\mathrm{~m}, 4 \mathrm{H}), 7.14-7.10(\mathrm{~m}, 2 \mathrm{H}), 7.06-7.00$ (m, 2H), $6.79-6.75(\mathrm{~m}, 2 \mathrm{H}), 6.65-6.57(\mathrm{~m}, 1 \mathrm{H}), 6.45-6.41(\mathrm{~m}, 2 \mathrm{H}), 5.34(\mathrm{~s}, 1 \mathrm{H}), 4.06(\mathrm{~s}$, $1 \mathrm{H}), 3.70(\mathrm{~s}, 3 \mathrm{H}) \mathrm{ppm}$.

${ }^{13}$ C NMR (151 MHz, Chloroform- $d$ ): $\delta=159.0,147.1,141.6,134.8,132.9,129.1,128.8$, $128.69,128.67,117.8,114.2,113.5,61.8,55.3 \mathrm{ppm}$.

HRMS (ESI): $m / z$ : $[\mathrm{M}+\mathrm{Na}]^{+}$Calcd. $\mathrm{C}_{20} \mathrm{H}_{18} \mathrm{ClNNaO}^{+}: 346.0969$; Found: $346,0959$.

IR (KBr): 3407, 3049, 3009, 2955, 2836, 2324, 2181, 2075, 1906, 1775, 1601, 1500, 1426, $1309,1246,1175,1090,1031,955,906,809,734,692 \mathrm{~cm}^{-1}$. 


\section{$N-([1,1 '-B i p h e n y l]-4-y l(p h e n y l) m e t h y l) a n i l i n e ~(10 t)$}<smiles>c1ccc(NC(c2ccccc2)c2ccc(-c3ccccc3)cc2)cc1</smiles>

The titled compound 10t was synthesized according to the general procedure GP-1 on a 0.2 mmol scale and was obtained after silica gel column chromatography using $n$-hexane/EtOAc $40: 1 \rightarrow 20: 1$ as colorless oil $(75 \%, 50 \mathrm{mg})$.

${ }^{1}$ H NMR $(600 \mathrm{MHz}$, Chloroform- $d$ ): $\delta=7.52-7.43(\mathrm{~m}, 4 \mathrm{H}), 7.37-7.30(\mathrm{~m}, 6 \mathrm{H}), 7.28-7.22$ (m, 3H), $7.21-7.16(\mathrm{~m}, 1 \mathrm{H}), 7.08-7.02(\mathrm{~m}, 2 \mathrm{H}), 6.65-6.60(\mathrm{~m}, 1 \mathrm{H}), 6.51-6.46(\mathrm{~m}, 2 \mathrm{H})$, $5.46(\mathrm{~s}, 1 \mathrm{H}), 4.18(\mathrm{~s}, 1 \mathrm{H}) \mathrm{ppm}$.

${ }^{13}$ C NMR (151 MHz, Chloroform- $d$ ): $\delta=147.3,142.9,142.0,140.7,140.3,129.1,128.8,128.7$, $127.8,127.52,127.50,127.4,127.3,127.0,117.7,113.5,62.8 \mathrm{ppm}$.

HRMS (APCI): $m / z:[\mathrm{M}+\mathrm{H}]^{+}$Calcd. $\mathrm{C}_{25} \mathrm{H}_{22} \mathrm{~N}^{+}: 336.1746$; Found: 336.1755 .

IR (KBr): 3410, 3053, 3027, 2923, 2850, 2319, 2248, 2051, 1915, 1812, 1760, 1684, 1599 , 1496, 1450, 1425, 1313, 1266, 1180, 1097, 1070, 1028, 1005, 907, 846, 730, $694 \mathrm{~cm}^{-1}$.

\section{$N$-(Phenyl(m-tolyl)methyl)aniline (10u)}<smiles>Cc1cccc(C(Nc2ccccc2)c2ccccc2)c1</smiles>

The titled compound 10u was synthesized according to the general procedure GP-1 on a 0.2 mmol scale and was obtained after silica gel column chromatography using $n$-hexane/EtOAc $40: 1 \rightarrow 20: 1$ as colorless oil $(68 \%, 37 \mathrm{mg})$.

${ }^{1}$ H NMR (600 MHz, Chloroform- $d$ ): $\delta=7.30-7.26(\mathrm{~m}, 2 \mathrm{H}), 7.25-7.21(\mathrm{~m}, 2 \mathrm{H}), 7.18-7.10$ (m, 2H), $7.10-7.05(\mathrm{~m}, 2 \mathrm{H}), 7.05-7.00(\mathrm{~m}, 2 \mathrm{H}), 7.00-6.96(\mathrm{~m}, 1 \mathrm{H}), 6.63-6.57(\mathrm{~m}, 1 \mathrm{H})$, $6.48-6.41(\mathrm{~m}, 2 \mathrm{H}), 5.37(\mathrm{~s}, 1 \mathrm{H}), 4.13(\mathrm{~s}, 1 \mathrm{H}), 2.23(\mathrm{~s}, 3 \mathrm{H}) \mathrm{ppm}$.

${ }^{13}$ C NMR (151 MHz, Chloroform- $d$ ): $\delta=147.5,143.08,143.02,138.4,129.1,128.7,128.6$, $128.19,128.17,127.4,127.3,124.5,117.6,113.5,63.1,21.5 \mathrm{ppm}$.

HRMS (ESI): $m / z$ : $[\mathrm{M}+\mathrm{Na}]^{+}$Calcd. $\mathrm{C}_{20} \mathrm{H}_{19} \mathrm{NNa}^{+}: 296.1409$; Found: 296.1403.

IR (KBr): 3409, 3025, 2919, 2857, 2332, 2019, 1940, 1813, 1686, 1599, 1497, 1451, 1425, $1312,1268,1179,1155,1067,1028,993,908,871,824,778,742,696 \mathrm{~cm}^{-1}$. 


\section{$N$-(Phenyl(o-tolyl)methyl)aniline (10v)}<smiles>Cc1ccccc1C(Nc1ccccc1)c1ccccc1</smiles>

The titled compound 10v was synthesized according to the general procedure GP- 1 on a 0.2 mmol scale and was obtained after silica gel column chromatography using $n$-hexane/EtOAc $40: 1 \rightarrow 20: 1$ as colorless solid $(82 \%, 45 \mathrm{mg})$.

${ }^{1}$ H NMR $(600 \mathrm{MHz}$, Chloroform- $d$ ): $\delta=7.38-7.31(\mathrm{~m}, 5 \mathrm{H}), 7.33-7.27(\mathrm{~m}, 1 \mathrm{H}), 7.24-7.16$ $(\mathrm{m}, 3 \mathrm{H}), 7.17-7.11(\mathrm{~m}, 2 \mathrm{H}), 6.74-6.68(\mathrm{~m}, 1 \mathrm{H}), 6.56-6.48(\mathrm{~m}, 2 \mathrm{H}), 5.69(\mathrm{~d}, J=3.4 \mathrm{~Hz}$, $1 \mathrm{H}), 4.16(\mathrm{~s}, 1 \mathrm{H}), 2.35$ (s, 3H) ppm.

${ }^{13}$ C NMR (151 MHz, Chloroform- $d$ ): $\delta=147.3,142.0,140.4,135.9,130.7,129.1,128.6,128.0$, 127.3, 127.29, 127.21, 126.3, 117.5, 113.1, 59.4, $19.4 \mathrm{ppm}$.

HRMS (ESI): $m / z$ : $[\mathrm{M}+\mathrm{Na}]^{+}$Calcd. $\mathrm{C}_{20} \mathrm{H}_{19} \mathrm{NNa}^{+}: 296.1409$; Found: 296.1401.

IR (KBr): 3411, 3053, 3024, 2921, 2331, 2159, 1919, 1815, 1764, 1599, 1497, 1454, 1425, $1380,1311,1268,1243,1179,1157,1120,1028,991,948,908,870,823,786,743,695 \mathrm{~cm}^{-1}$.

\section{$N$-((2-Chlorophenyl)(phenyl)methyl)aniline (10w)}<smiles>Clc1ccccc1C(Nc1ccccc1)c1ccccc1</smiles>

The titled compound 10w was synthesized according to the general procedure GP-1 on a 0.2 mmol scale and was obtained after silica gel column chromatography using $n$-hexane/EtOAc $40: 1 \rightarrow 20: 1$ as colorless oil $(63 \%, 37 \mathrm{mg})$.

${ }^{1}$ H NMR (600 MHz, Chloroform- $d$ ): $\delta=7.50-7.45(\mathrm{~m}, 1 \mathrm{H}), 7.33-7.28(\mathrm{~m}, 1 \mathrm{H}), 7.27-7.23$ $(\mathrm{m}, 4 \mathrm{H}), 7.23-7.12(\mathrm{~m}, 3 \mathrm{H}), 7.07-7.02(\mathrm{~m}, 2 \mathrm{H}), 6.65-6.60(\mathrm{~m}, 1 \mathrm{H}), 6.45-6.39(\mathrm{~m}, 2 \mathrm{H})$, $5.83(\mathrm{~s}, 1 \mathrm{H}), 4.13(\mathrm{~s}, 1 \mathrm{H}) \mathrm{ppm}$.

${ }^{13}$ C NMR (151 MHz, Chloroform- $d$ ): $\delta=147.0,141.4$, 139.6, 133.5, 129.9, 129.2, 128.7, $128.63,128.60,128.0,127.6,127.2,117.9,113.3,59.5 \mathrm{ppm}$.

HRMS (ESI): $m / z:[\mathrm{M}+\mathrm{H}]^{+}$Calcd. $\mathrm{C}_{19} \mathrm{H}_{17} \mathrm{ClN}^{+}: 294.1044$; Found: 294.1036.

IR (KBr): 3412, 3056, 2921, 2554, 2160, 1923, 1814, 1763, 1599, 1498, 1443, 1312, 1260 , $1183,1156,1130,1100,1071,1036,992,950,907,871,821,746,694 \mathrm{~cm}^{-1}$. 


\section{$N$-((4-methoxyphenyl)(3-nitrophenyl)methyl)aniline (10x)}<smiles>COc1ccc(C(Nc2ccccc2)c2cccc([N+](=O)[O-])c2)cc1</smiles>

The titled compound 10x was synthesized according to the general procedure GP-1. And was obtained after silica gel column chromatography using n-hexane/EtOAc 10:1 $\rightarrow$ 4:1 as yellow oil (97\%, $65 \mathrm{mg})$.

${ }^{1}$ H NMR (600 MHz, Chloroform- $d$ ): $\delta=8.19-8.14(\mathrm{~m}, 1 \mathrm{H}), 8.05-7.99(\mathrm{~m}, 1 \mathrm{H}), 7.69-7.62$ (m, 1H), $7.41(\mathrm{t}, J=7.9 \mathrm{~Hz}, 1 \mathrm{H}), 7.15-7.11(\mathrm{~m}, 2 \mathrm{H}), 7.10-7.01(\mathrm{~m}, 2 \mathrm{H}), 6.82-6.75(\mathrm{~m}$, $2 \mathrm{H}), 6.69-6.63(\mathrm{~m}, 1 \mathrm{H}), 6.47-6.40(\mathrm{~m}, 2 \mathrm{H}), 5.45(\mathrm{~s}, 1 \mathrm{H}), 4.14(\mathrm{~s}, 1 \mathrm{H}), 3.71(\mathrm{~s}, 3 \mathrm{H}) \mathrm{ppm}$.

${ }^{13}$ C NMR (151 MHz, Chloroform- $d$ ): $\delta=159.3,148.7,146.7,145.5,134.0,133.2,129.7,129.2$, $128.8,122.3,122.1,118.3,114.5,113.6,62.0,55.3 \mathrm{ppm}$.

HRMS (ESI): $m / z$ : $[\mathrm{M}+\mathrm{Na}]^{+}$Calcd. $\mathrm{C}_{20} \mathrm{H}_{18} \mathrm{~N}_{2} \mathrm{O}_{3} \mathrm{Na}^{+}$: 357.1209; Found: 357.1198 .

IR (KBr): 3403, 3052, 2934, 2838, 2249, 2187, 2055, 1919, 1601, 1503, 1432, 1347, 1311, $1247,1176,1097,1030,908,874,834,807,730,689 \mathrm{~cm}^{-1}$.

\section{$N$-Benzhydryl- $N$-methylaniline (12a)}<smiles>CN(c1ccccc1)C(c1ccccc1)c1ccccc1</smiles>

The titled compound 12a was synthesized according to the general procedure GP-2 on a 0.2 mmol scale and was obtained after silica gel column chromatography using $n$-hexane/EtOAc $40: 1 \rightarrow 20: 1$ as colorless oil $(66 \%, 36 \mathrm{mg})$.

${ }^{1}$ H NMR (600 MHz, Chloroform- $d$ ): $\delta=7.27-7.22(\mathrm{~m}, 4 \mathrm{H}), 7.22-7.18(\mathrm{~m}, 2 \mathrm{H}), 7.16-7.10$ $(\mathrm{m}, 6 \mathrm{H}), 6.74-6.70(\mathrm{~m}, 2 \mathrm{H}), 6.68-6.62(\mathrm{~m}, 1 \mathrm{H}), 6.11(\mathrm{~s}, 1 \mathrm{H}), 2.66(\mathrm{~s}, 3 \mathrm{H}) \mathrm{ppm}$.

${ }^{13}$ C NMR (151 MHz, Chloroform- $d$ ): $\delta=150.2,140.7,129.1,128.7,128.4,127.2,116.8,112.9$, 67.0, $34.5 \mathrm{ppm}$.

HRMS (ESI): $m / z$ : $[\mathrm{M}+\mathrm{H}]^{+}$Calcd. for $\mathrm{C}_{20} \mathrm{H}_{20} \mathrm{~N}^{+}$: 274.1590; Found: 274.1587 .

IR (KBr): 3060, 3026, 2947, 2873, 2793, 2162, 1950, 1897, 1741, 1597, 1492, 1449, 1368, $1296,1242,1163,1082,1030,979,949,914,864,832,766,728,698 \mathrm{~cm}^{-1}$. 


\section{$N$-Benzhydryl- $N$-ethylaniline (12b)}<smiles>CCN(c1ccccc1)C(c1ccccc1)c1ccccc1</smiles>

The titled compound 12b was synthesized according to the general procedure GP-2 on a 0.2 mmol scale and was obtained after silica gel column chromatography using $n$-hexane/EtOAc $40: 1 \rightarrow 20: 1$ as colorless oil $(70 \%, 40 \mathrm{mg})$.

${ }^{1}$ H NMR (600 MHz, Chloroform- $d$ ): $\delta=7.24-7.20(\mathrm{~m}, 4 \mathrm{H}), 7.19-7.15(\mathrm{~m}, 2 \mathrm{H}), 7.14-7.07$ $(\mathrm{m}, 6 \mathrm{H}), 6.72-6.68(\mathrm{~m}, 2 \mathrm{H}), 6.65-6.58(\mathrm{~m}, 1 \mathrm{H}), 6.06(\mathrm{~s}, 1 \mathrm{H}), 3.28(\mathrm{q}, J=7.0 \mathrm{~Hz}, 2 \mathrm{H}), 0.72$ (t, $J=6.9 \mathrm{~Hz}, 3 \mathrm{H}) \mathrm{ppm}$.

${ }^{13}$ C NMR (151 MHz, Chloroform- $d$ ): $\delta=148.9,141.0,129.1,129.0,128.3,127.1,116.8,113.8$, 66.9, 42.2, $13.6 \mathrm{ppm}$.

HRMS (ESI): $m / z$ : $[\mathrm{M}+\mathrm{H}]^{+}$Calcd. for $\mathrm{C}_{21} \mathrm{H}_{22} \mathrm{~N}^{+}: 288.1746$; Found: 288.1741 .

IR (KBr): 3088, 3027, 2980, 2929, 2875, 2645, 2319, 2156, 1958, 1906, 1813, 1755, 1663 , 1594, 1496, 1449, 1372, 1334, 1265, 1200, 1162, 1113, 1075, 1029, 999, 934, 908, 861, 815, $770,737,692 \mathrm{~cm}^{-1}$.

\section{$N$-Benzhydryl-4-methoxy- $N$-methylaniline (12c)}<smiles>COc1ccc(N(C)C(c2ccccc2)c2ccccc2)cc1</smiles>

The titled compound 12c was synthesized according to the general procedure GP-2 on a 0.2 mmol scale and was obtained after silica gel column chromatography using $n$-hexane/EtOAc $20: 1 \rightarrow 10: 1$ as colorless oil $(70 \%, 43 \mathrm{mg})$.

${ }^{1}$ H NMR (600 MHz, Chloroform- $d$ ): $\delta=7.26-7.20(\mathrm{~m}, 4 \mathrm{H}), 7.20-7.15(\mathrm{~m}, 2 \mathrm{H}), 7.16-7.11$ (m, 4H), $6.74-6.71(\mathrm{~m}, 2 \mathrm{H}), 6.70-6.66(\mathrm{~m}, 2 \mathrm{H}), 5.91(\mathrm{~s}, 1 \mathrm{H}), 3.67(\mathrm{~s}, 3 \mathrm{H}), 2.59(\mathrm{~s}, 3 \mathrm{H}) \mathrm{ppm}$. ${ }^{13}$ C NMR (151 MHz, Chloroform- $d$ ): $\delta=152.0,145.1,141.0,128.7,128.3,127.0,115.6,114.6$, $68.7,55.7,35.5 \mathrm{ppm}$.

HRMS (ESI): $m / z$ : $[\mathrm{M}+\mathrm{H}]^{+}$Calcd. for $\mathrm{C}_{21} \mathrm{H}_{22} \mathrm{NO}^{+}:$304.1695; Found: 304.1695 .

IR (KBr): 3413, 3055, 3022, 2891, 2822, 2330, 2160, 2087, 1955, 1889, 1854, 1813, 1739 , 1592, 1495, 1448, 1361, 1314, 1273, 1243, 1219, 1178, 1103, 1077, 1030, 999, 954, 914, 869, $801,735,697 \mathrm{~cm}^{-1}$. 


\section{$N$-Benzhydryl-4-chloro- $N$-methylaniline (12d)}<smiles>CN(c1ccc(Cl)cc1)C(c1ccccc1)c1ccccc1</smiles>

The titled compound 12d was synthesized according to the general procedure GP-2 on a 0.2 mmol scale and was obtained after silica gel column chromatography using $n$-hexane/EtOAc $40: 1 \rightarrow 20: 1$ as colorless oil $(91 \%, 56 \mathrm{mg})$.

${ }^{1}$ H NMR $(600 \mathrm{MHz}$, Chloroform- $d$ ): $\delta=7.27-7.22(\mathrm{~m}, 4 \mathrm{H}), 7.22-7.18(\mathrm{~m}, 2 \mathrm{H}), 7.10-7.04$ (m, 6H), $6.61(\mathrm{~d}, J=9.1 \mathrm{~Hz}, 2 \mathrm{H}), 6.02(\mathrm{~s}, 1 \mathrm{H}), 2.63(\mathrm{~s}, 3 \mathrm{H}) \mathrm{ppm}$.

${ }^{13}$ C NMR (151 MHz, Chloroform- $d$ ): $\delta=148.7,140.3,128.9,128.7,128.5,127.3,121.6,114.1$, $67.3,34.7 \mathrm{ppm}$.

HRMS (ESI): $m / z$ : $[\mathrm{M}]^{+}$Calcd. for $\mathrm{C}_{20} \mathrm{H}_{19} \mathrm{ClN}^{+}: 307.1122$; Found: 307.1124 .

IR (KBr): 3056, 3024, 2999, 2933, 2889, 2824, 2326, 2157, 2076, 1954, 1889, 1813, 1759 , 1595, 1507, 1448, 1367, 1311, 1241, 1178, 1105, 1076, 1039, 960, 912, 868, 810, 766, 727, $699 \mathrm{~cm}^{-1}$.

$N$-Benzhydryl- $N, 3-$ dimethylaniline (12e)<smiles>Cc1cccc(N(C)C(c2ccccc2)c2ccccc2)c1</smiles>

The titled compound 12e was synthesized according to the general procedure GP-2 on a 0.2 mmol scale and was obtained after silica gel column chromatography using $n$-hexane/EtOAc $40: 1 \rightarrow 20: 1$ as colorless oil $(45 \%, 26 \mathrm{mg})$.

${ }^{1}$ H NMR (600 MHz, Chloroform- $d$ ): $\delta=7.27-7.23(\mathrm{~m}, 3 \mathrm{H}), 7.22-7.18(\mathrm{~m}, 3 \mathrm{H}), 7.14-7.10$ (m, 4H), $7.03(\mathrm{t}, J=7.8 \mathrm{~Hz}, 1 \mathrm{H}), 6.57-6.52(\mathrm{~m}, 2 \mathrm{H}), 6.49(\mathrm{~d}, J=7.4 \mathrm{~Hz}, 1 \mathrm{H}), 6.12(\mathrm{~s}, 1 \mathrm{H})$, $2.64(\mathrm{~s}, 3 \mathrm{H}), 2.22(\mathrm{~s}, 3 \mathrm{H}) \mathrm{ppm}$.

${ }^{13}$ C NMR (151 MHz, Chloroform- $d$ ): $\delta=150.3,140.7,138.8,128.9,128.7,128.3,127.1,117.7$, 113.6, 110.2, 67.0, 34.4, $21.9 \mathrm{ppm}$.

HRMS (ESI): $m / z$ : $[\mathrm{M}+\mathrm{H}]^{+}$Calcd. for $\mathrm{C}_{21} \mathrm{H}_{22} \mathrm{~N}^{+}: 288.1746$; Found: 288.1743 .

IR (KBr): 3418, 3027, 2918, 2815, 2329, 2161, 2092, 1948, 1905, 1810, 1736, 1660, 1599 , $1493,1449,1367,1314,1224,1178,1107,1001,963,919,835,765,727,696 \mathrm{~cm}^{-1}$. 
<smiles>Cc1ccccc1N(C)C(c1ccccc1)c1ccccc1</smiles>

The titled compound $\mathbf{1 2 f}$ was synthesized according to the general procedure GP-2 on a 0.2 mmol scale and was obtained after silica gel column chromatography using $n$-hexane/EtOAc $40: 1 \rightarrow 20: 1$ as colorless oil $(49 \%, 28 \mathrm{mg})$.

${ }^{1}$ H NMR (600 MHz, Chloroform- $d$ ): $\delta=7.30-7.24(\mathrm{~m}, 4 \mathrm{H}), 7.19-7.13(\mathrm{~m}, 4 \mathrm{H}), 7.10-7.05$ $(\mathrm{m}, 3 \mathrm{H}), 6.92-6.87(\mathrm{~m}, 1 \mathrm{H}), 6.85-6.80(\mathrm{~m}, 1 \mathrm{H}), 6.79-6.73(\mathrm{~m}, 1 \mathrm{H}), 5.25(\mathrm{~s}, 1 \mathrm{H}), 2.41(\mathrm{~s}$, $3 \mathrm{H}), 2.37$ (s, 3H) ppm.

${ }^{13}$ C NMR (151 MHz, Chloroform- $d$ ): $\delta=151.2,142.0,133.8,130.9,128.2,128.1,126.7,125.9$, 123.2, 122.5, 72.4, 40.3, $18.3 \mathrm{ppm}$.

HRMS (ESI): $m / z$ : $[\mathrm{M}]^{+}$Calcd. for $\mathrm{C}_{21} \mathrm{H}_{21} \mathrm{~N}^{+}: 287.1668$; Found: 287.1673.

IR (KBr): 3414, 3195, 3059, 3026, 2885, 2816, 2325, 2106, 1997, 1904, 1813, 1660, 1596, 1498, 1449, 1367, 1317, 1219, 1189, 1162, 1105, 1077, 1030, 989, 956, 917, 862, 833, 797, $741,695 \mathrm{~cm}^{-1}$.

$N$-Benzhydryl- $N$-phenylaniline (12g)<smiles>c1ccc(C(c2ccccc2)N(c2ccccc2)c2ccccc2)cc1</smiles>

The titled compound $\mathbf{1 2 g}$ was synthesized according to the general procedure GP-2 on a 0.2 mmol scale and was obtained after silica gel column chromatography using $n$-hexane/EtOAc $40: 1 \rightarrow 20: 1$ as colorless oil $(48 \%, 32 \mathrm{mg})$.

${ }^{1}$ H NMR (600 MHz, Chloroform- $d$ ): $\delta=7.30-7.27(\mathrm{~m}, 4 \mathrm{H}), 7.26-7.22(\mathrm{~m}, 4 \mathrm{H}), 7.21-7.18$ (m, 2H), $7.17-7.12(\mathrm{~m}, 4 \mathrm{H}), 6.92-6.82(\mathrm{~m}, 6 \mathrm{H}), 6.41(\mathrm{~s}, 1 \mathrm{H}) \mathrm{ppm}$.

${ }^{13}$ C NMR (151 MHz, Chloroform- $d$ ): $\delta=147.2,141.1,129.4,128.7,128.0,126.9,123.3,121.6$, $68.9 \mathrm{ppm}$.

HRMS (ESI): $m / z$ : $[\mathrm{M}+\mathrm{Na}]^{+}$Calcd. for $\mathrm{C}_{25} \mathrm{H}_{21} \mathrm{NNa}^{+}: 358,1566$; Found: 358.1561 .

IR (KBr): 3398, 3026, 2925, 2324, 2111, 1893, 1821, 1735, 1587, 1490, 1447, 1393, 1312 , $1239,1165,1076,1053,1027,993,916,865,792,743,695 \mathrm{~cm}^{-1}$. 


\section{References}

[1] Yang, L.-L.; Evans, D.; Xu, B.; Li, W.-T.; Li, M.-L.; Zhu, S.-F.; Houk, K. N.; Zhou, Q.-L., Enantioselective Diarylcarbene Insertion into $\mathrm{Si}-\mathrm{H}$ Bonds Induced by Electronic Properties of the Carbenes. J. Am. Chem. Soc. 2020, 142, 12394-12399.

[2] Lee, M.; Ren, Z.; Musaev, D. G.; Davies, H. M. L. Rhodium-Stabilized Diarylcarbenes Behaving as Donor/Acceptor Carbenes. ACS Catal. 2020, 10, 6240-6247.

[3] Wu, C.; Hu, F.; Liu, Z.; Deng, G.; Ye, F.; Zhang, Y.; Wang, J, Cu(I)-catalyzed coupling of diaryldiazomethanes with terminalalkynes: an efficient synthesis of tri-aryl-substituted allenes. Tetrahedron, 2015, 71, 9696-9201.

[4] Jagannathan, J. R.; Fettinger, J. C.; Shaw, J. T.; Franz, A. J. K. Enantioselective Si-H Insertion Reactions of Diarylcarbenes for the Synthesis of Silicon-Stereogenic Silanes, J. Am. Chem. Soc. 2020, 142, 11674-11679. 


\section{Spectra}

1-(Diazo(phenyl)methyl)-3-methylbenzene (1e)

${ }^{1}$ H NMR (600 MHz, Chloroform-d)

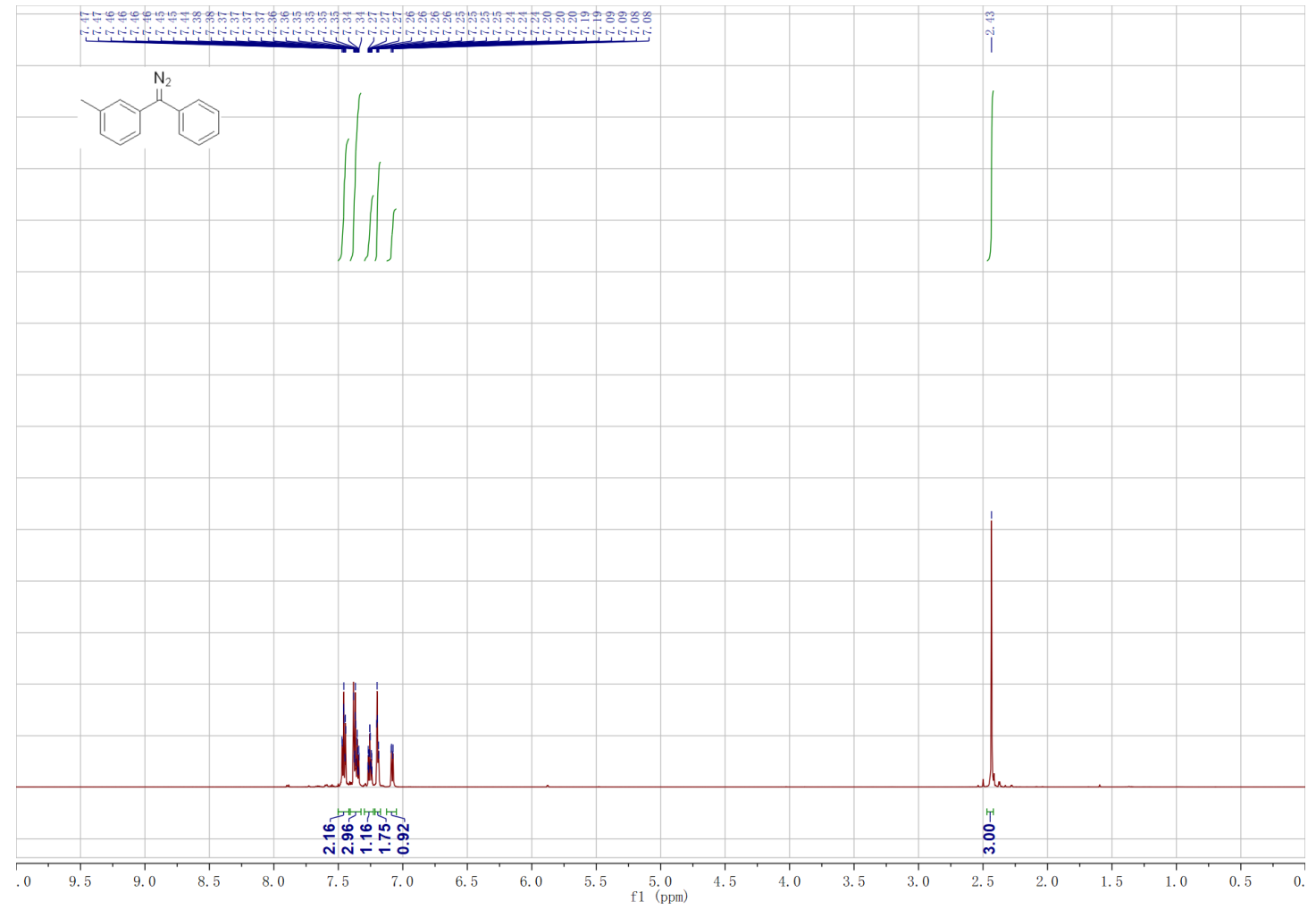

${ }^{13}$ C NMR (151 MHz, Chloroform-d)

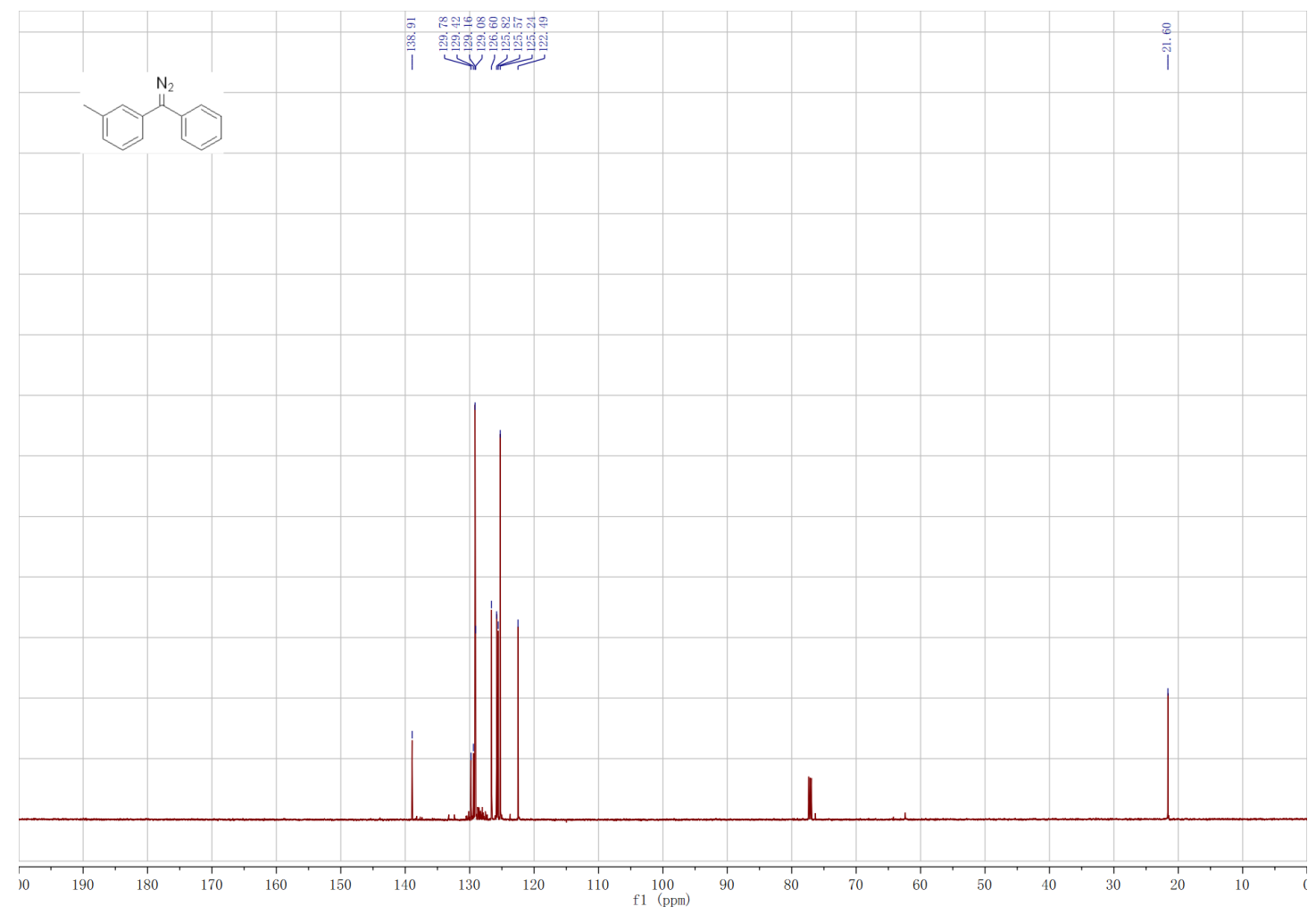


1-(Diazo(4-methoxyphenyl)methyl)-3-nitrobenzene (1h)

${ }^{1}$ H NMR (600 MHz, Chloroform- $d$ )

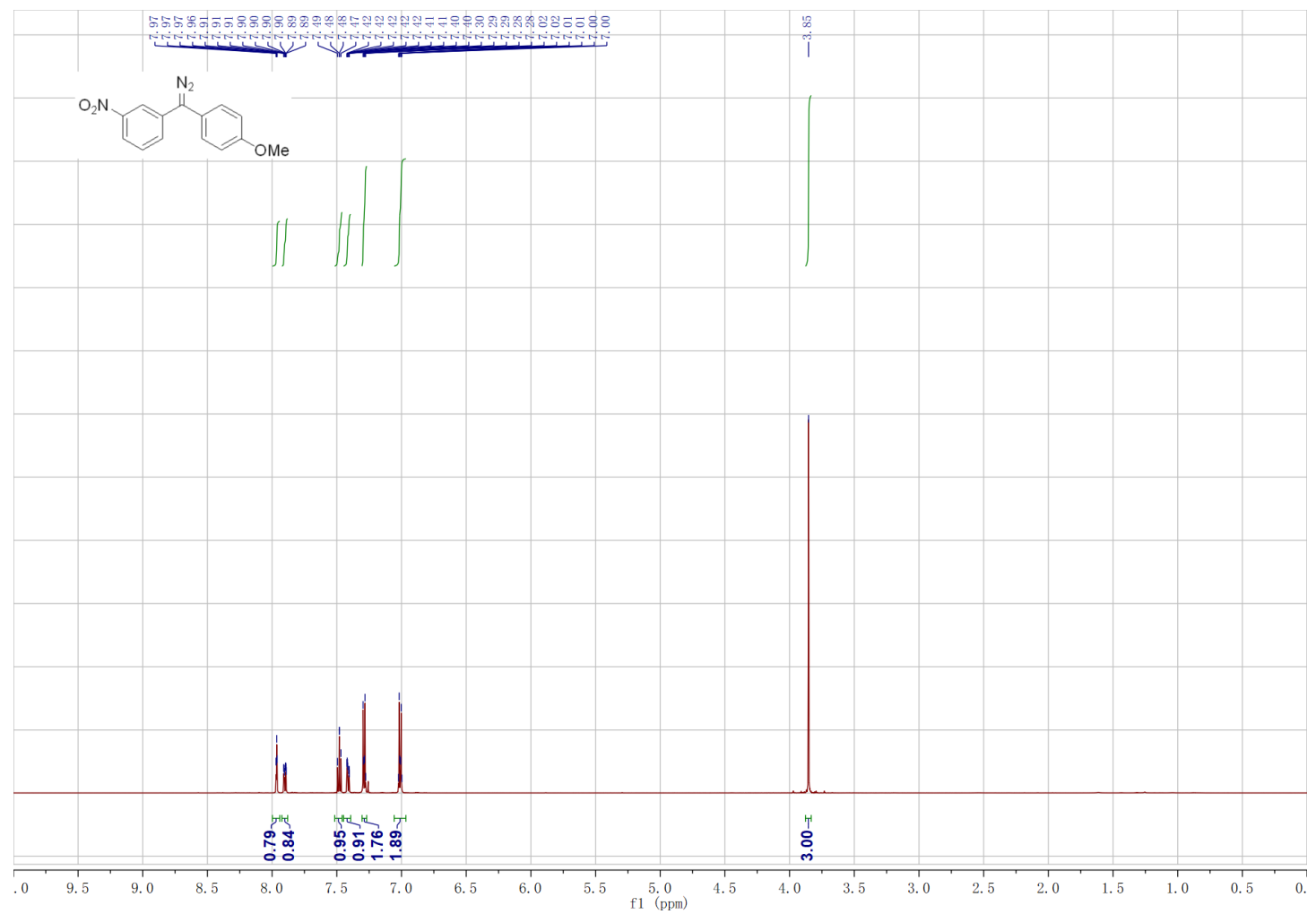

${ }^{13}$ C NMR (151 MHz, Chloroform- $d$ )

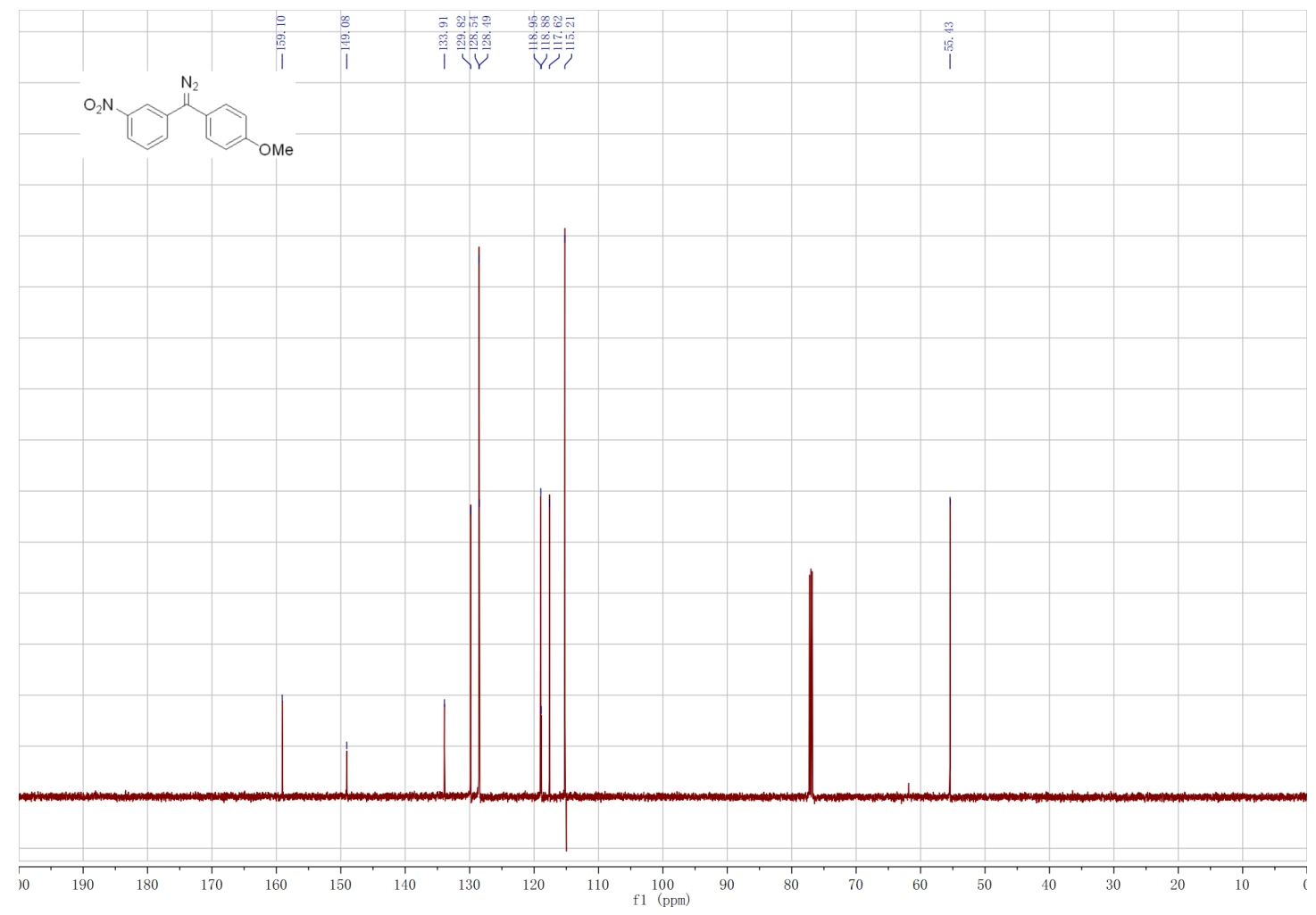


2-(Diazo(4-nitrophenyl)methyl)-1,4-dimethylbenzene (1j)

${ }^{1}$ H NMR (400 MHz, Chloroform- $d$ )

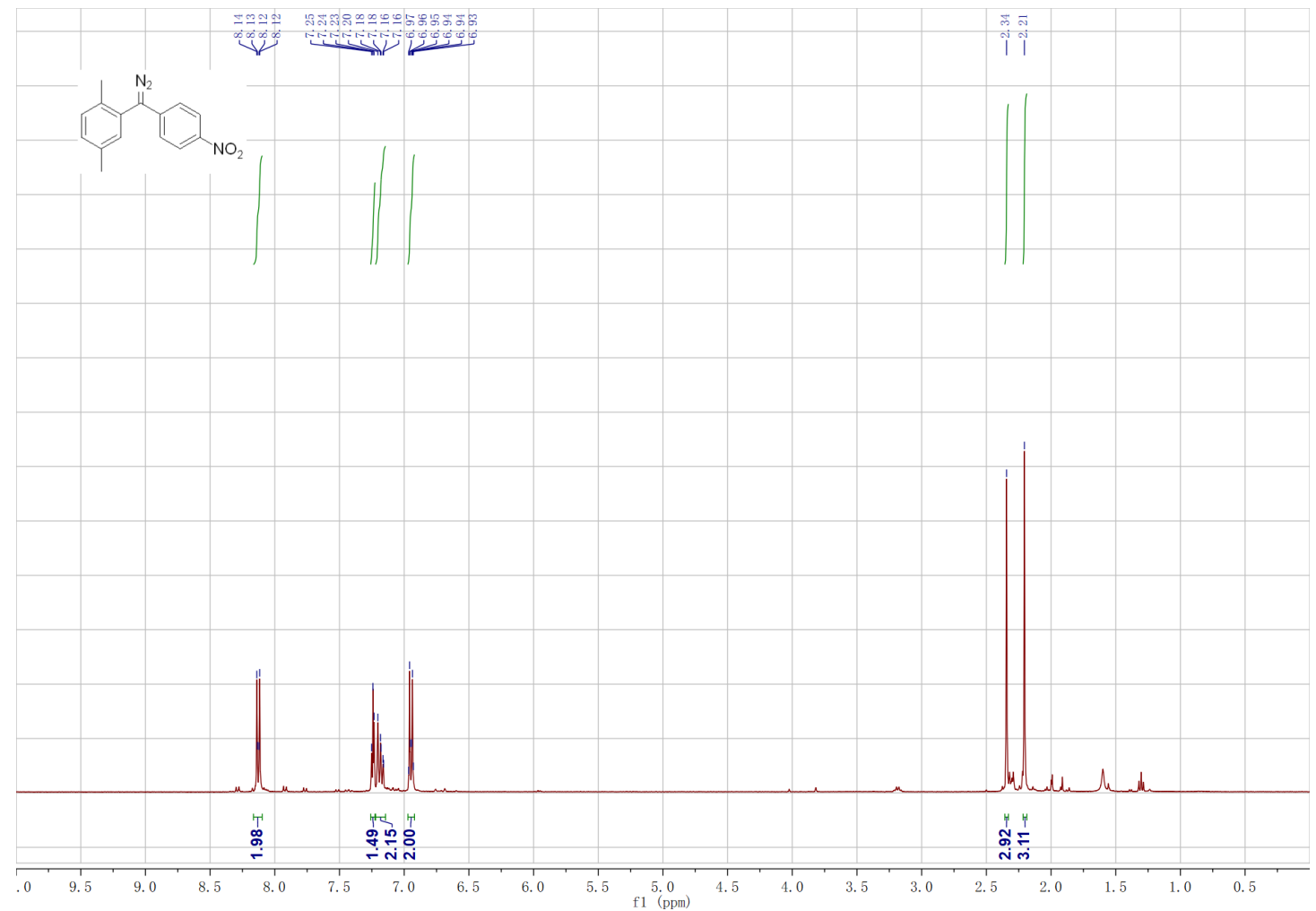

${ }^{13}$ C NMR (101 MHz, Chloroform- $d$ )

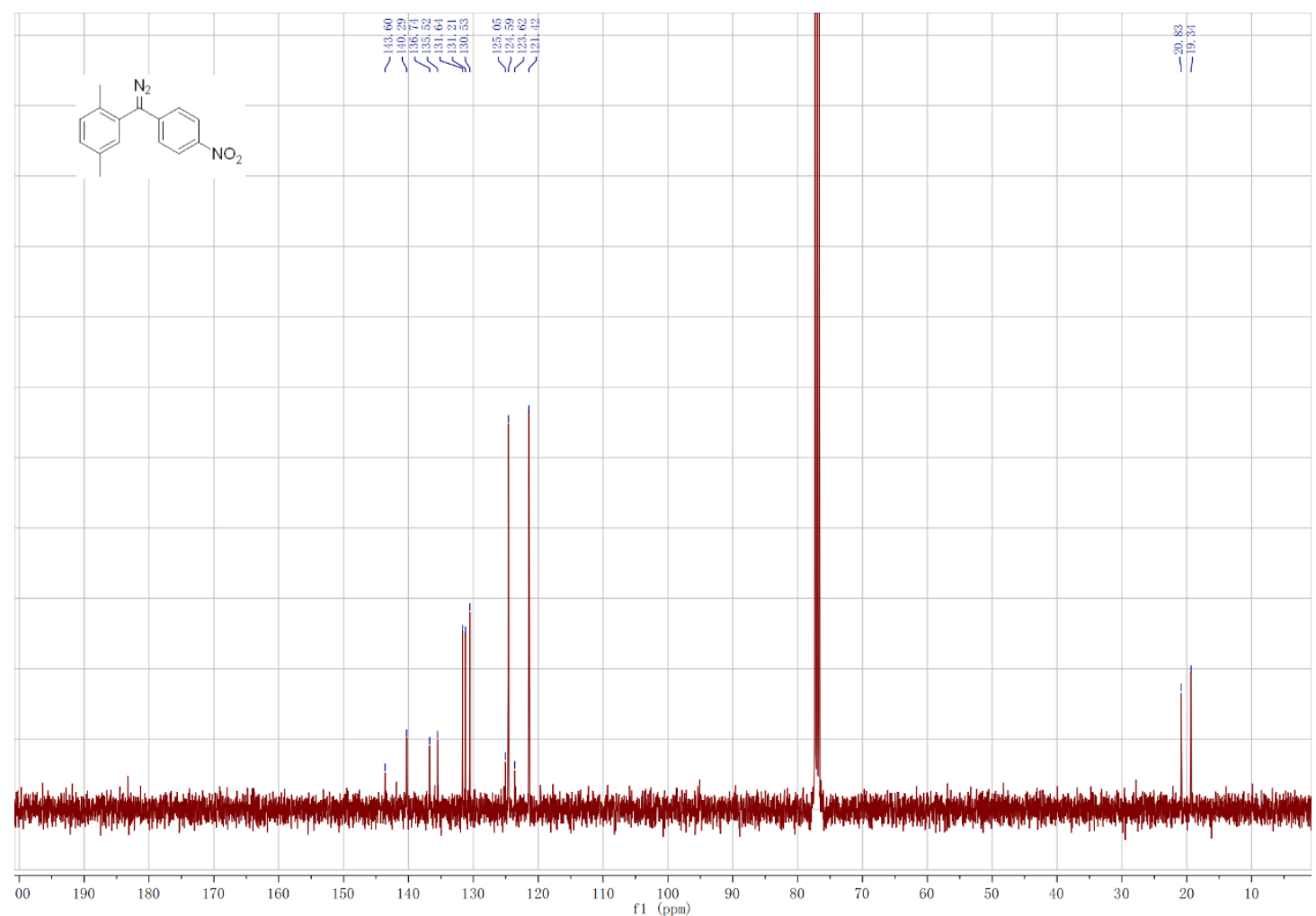


$N$-Benzhydrylaniline (10a)

${ }^{1} \mathrm{H}$ NMR (600 MHz, Chloroform- $d$ )

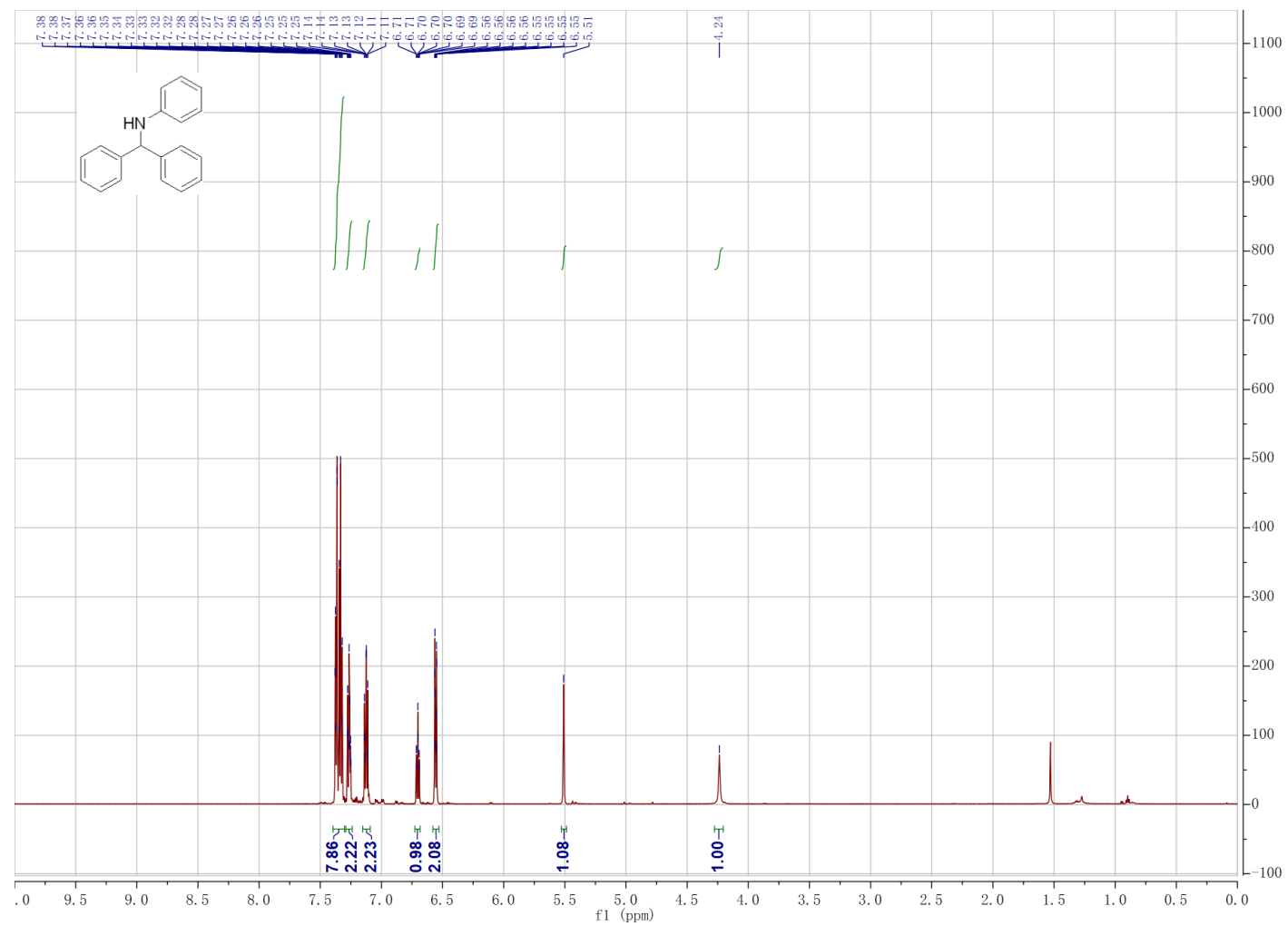

${ }^{13} \mathrm{C}$ NMR (151 MHz, Chloroform- $d$ )

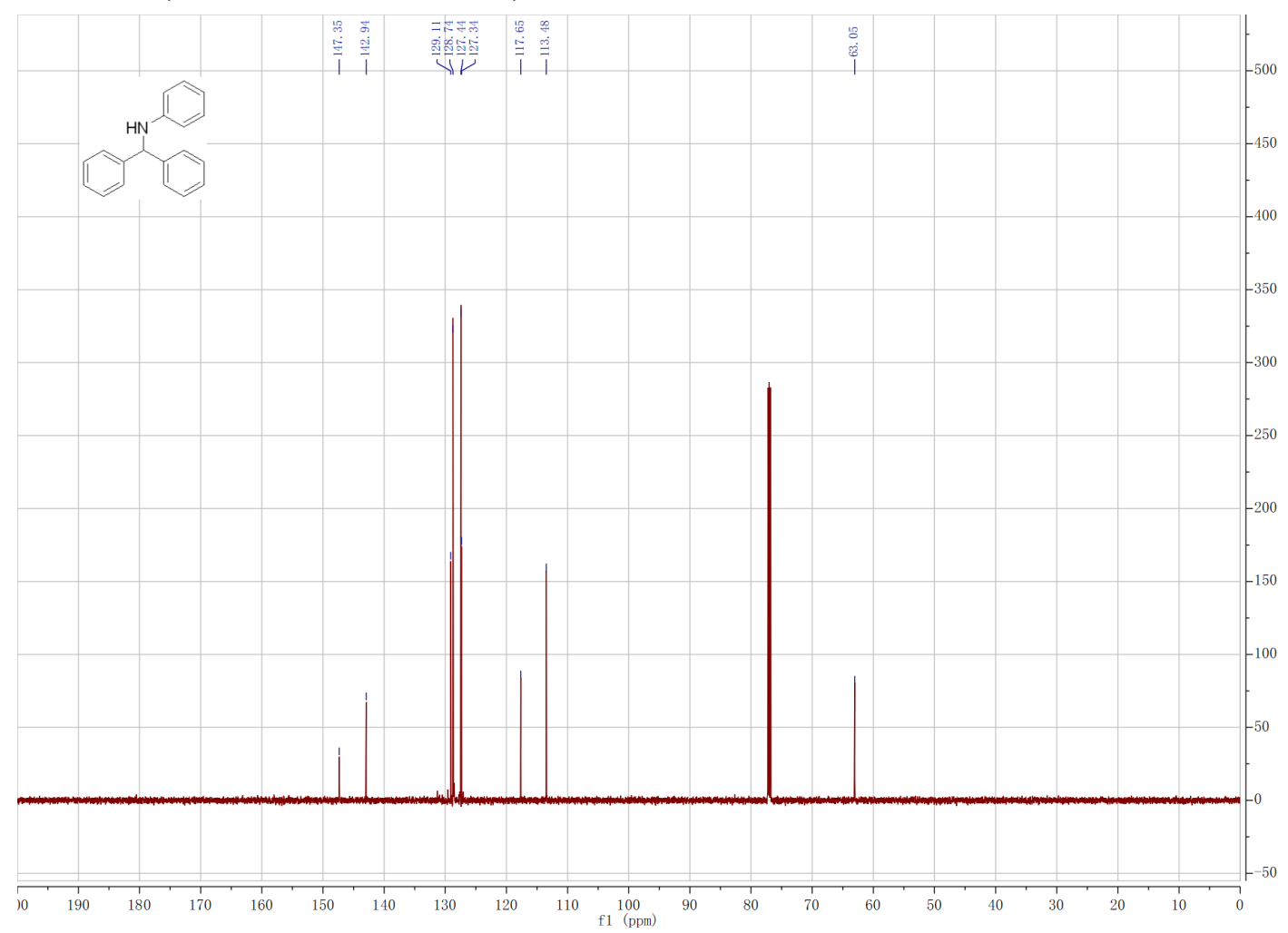


$\mathrm{N}$-Benzhydryl-4-methylaniline (10b)

${ }^{1} \mathrm{H}$ NMR (600 MHz, Chloroform- $d$ )

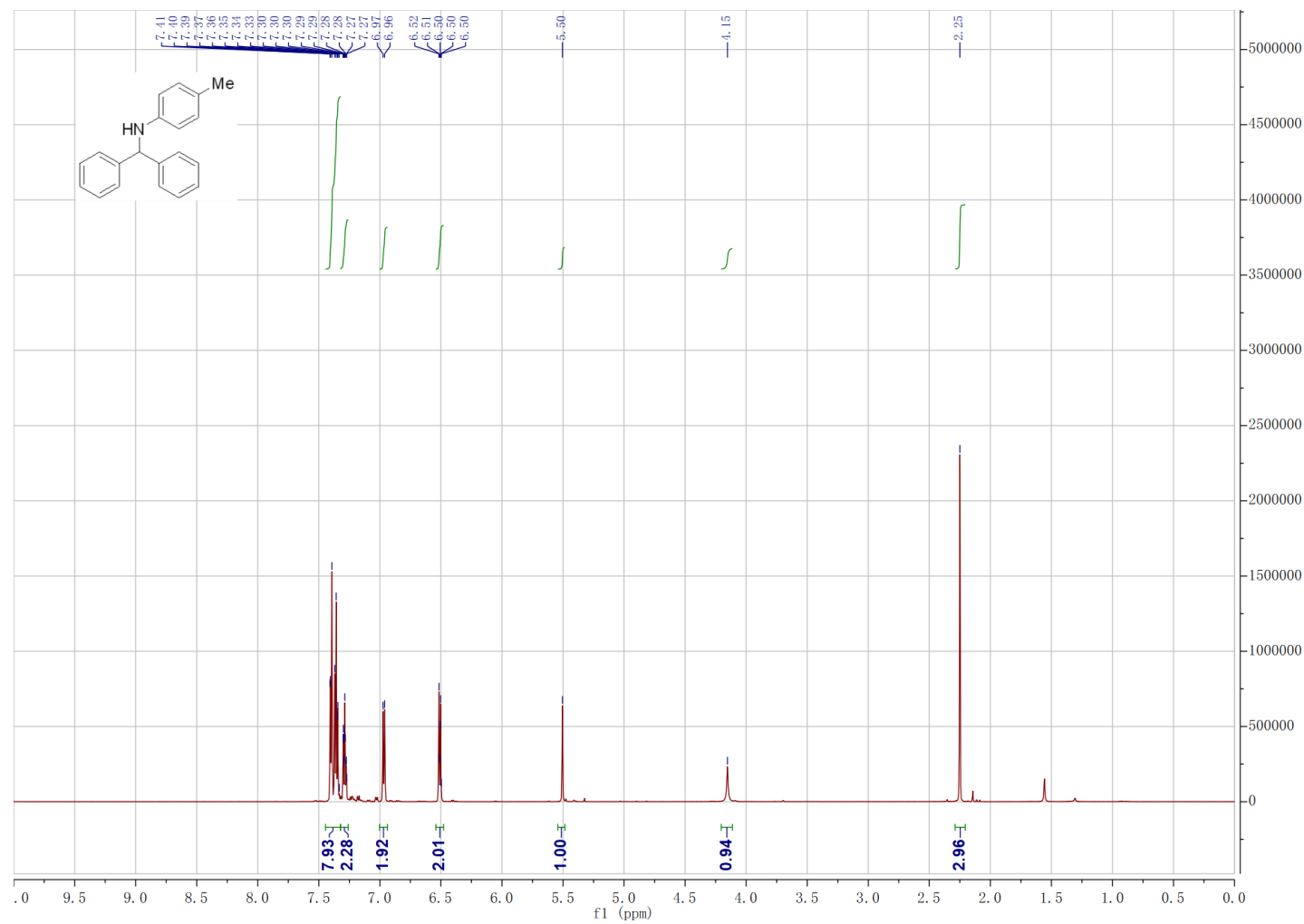

${ }^{13} \mathrm{C}$ NMR (151 MHz, Chloroform- $d$ )

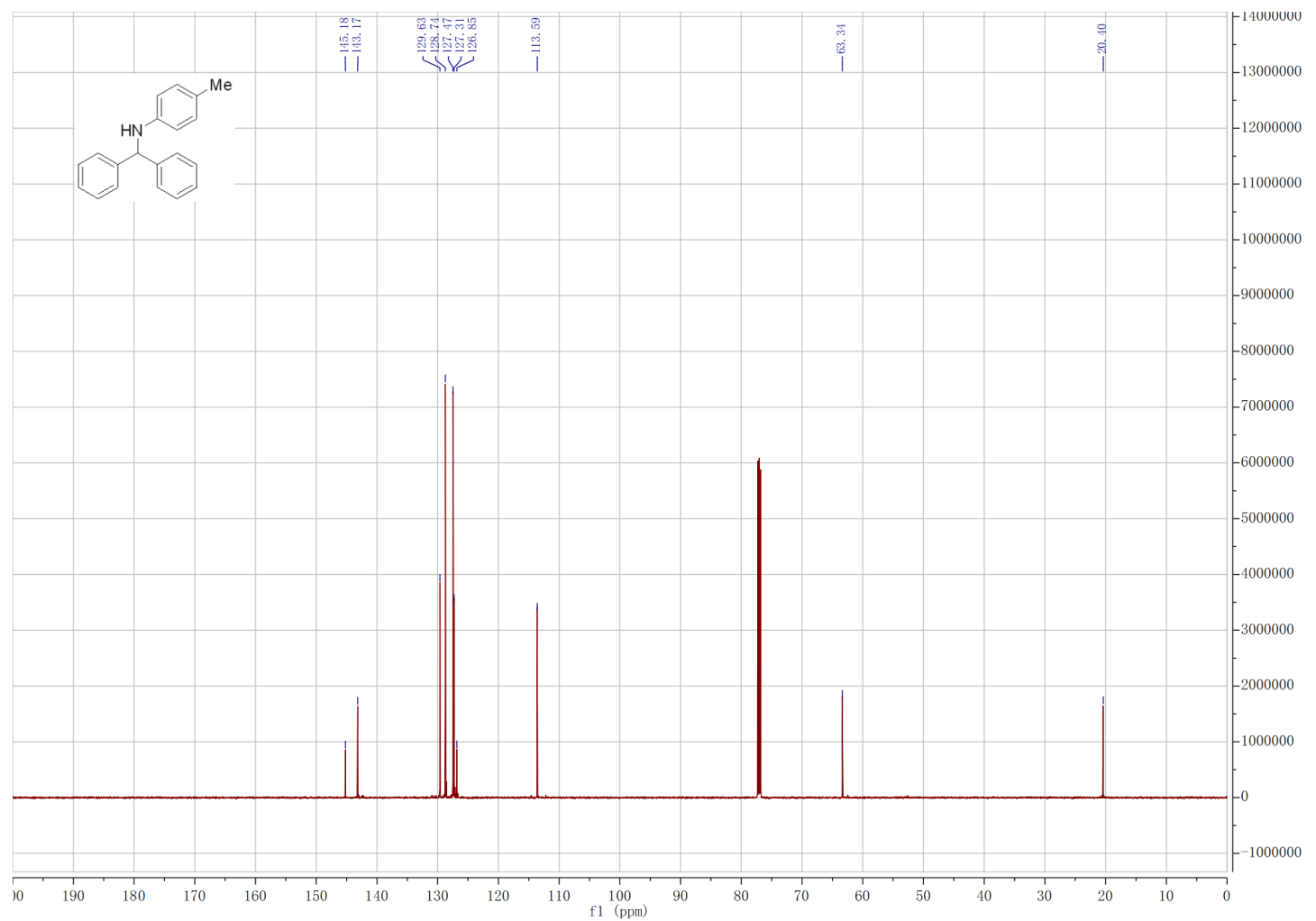


$N$-Benzhydryl-4-methoxyaniline (10c)

${ }^{1} \mathrm{H}$ NMR (600 MHz, Chloroform- $d$ )

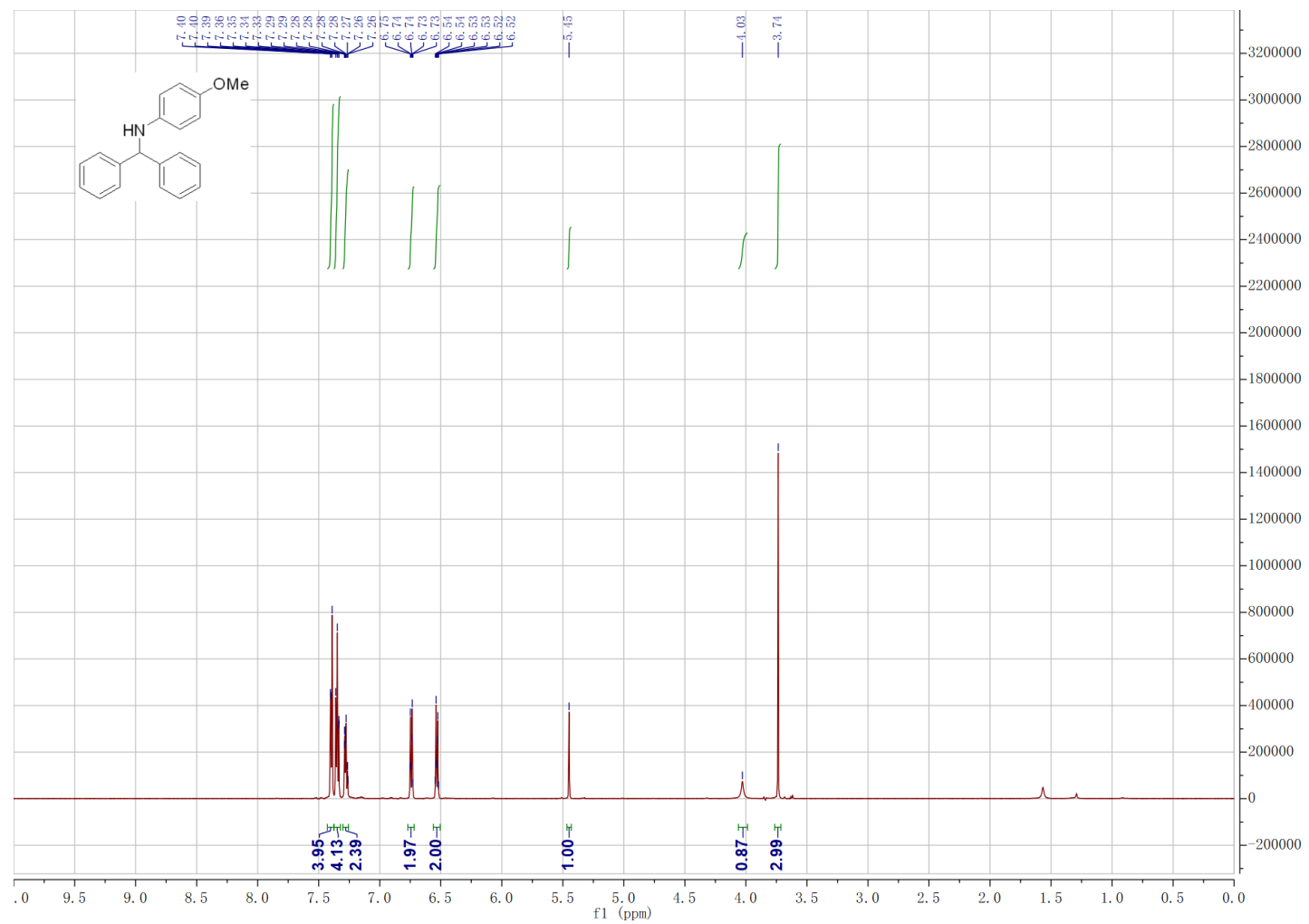

${ }^{13} \mathrm{C}$ NMR (151 MHz, Chloroform- $d$ )

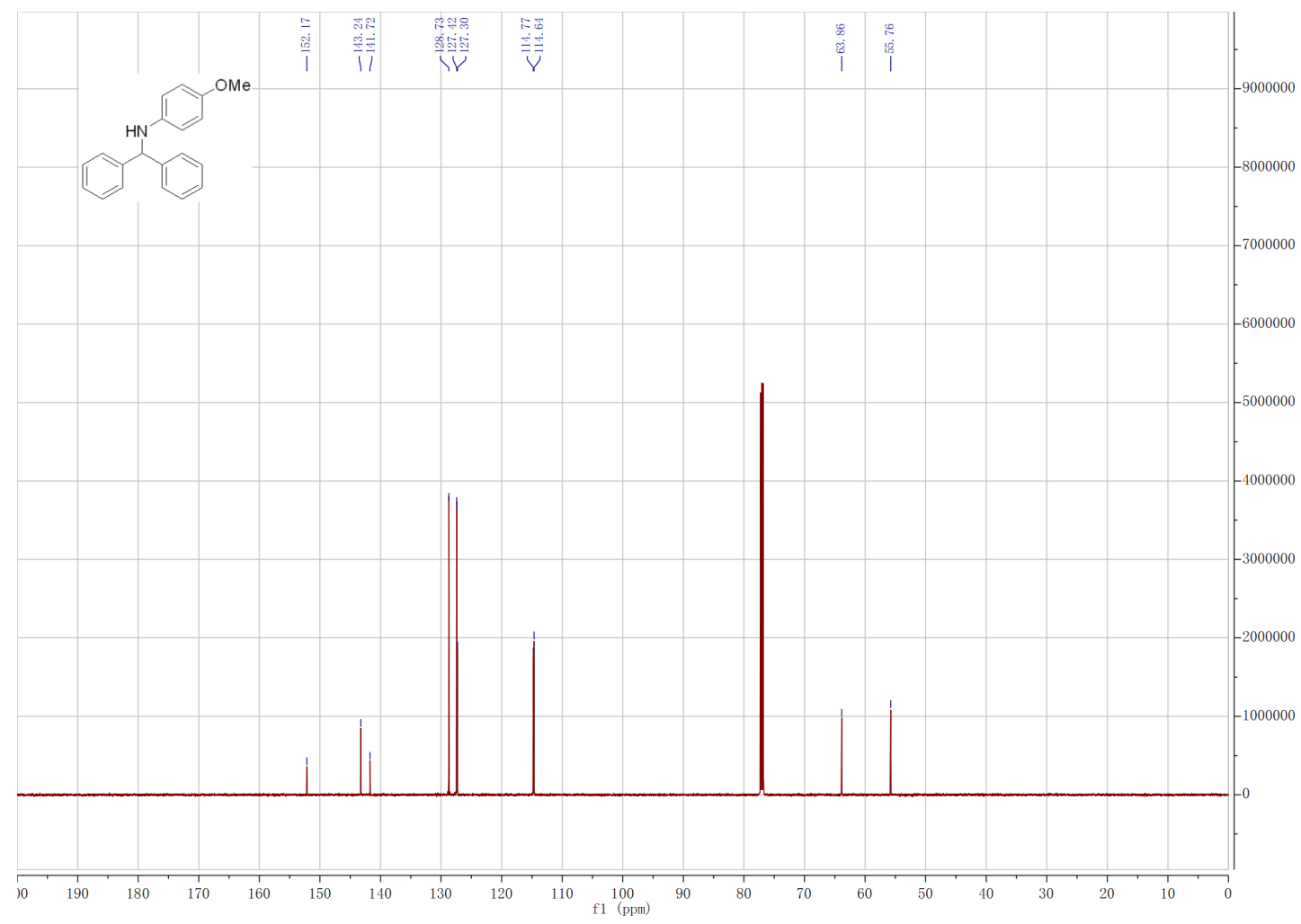


$N$-Benzhydryl-4-fluoroaniline (10d)

${ }^{1} \mathrm{H}$ NMR (600 MHz, Chloroform- $d$ )

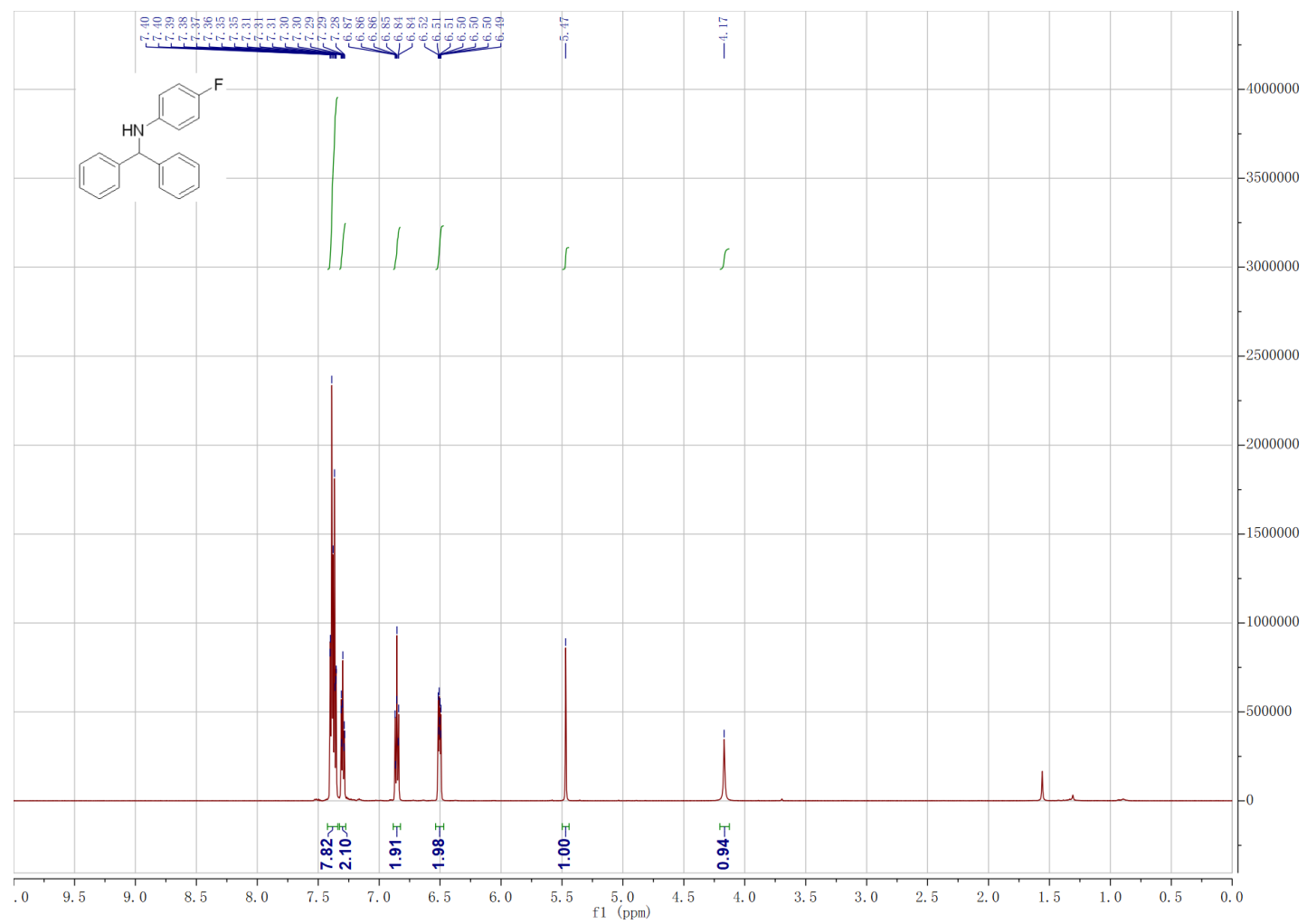

${ }^{13} \mathrm{C}$ NMR (151 MHz, Chloroform- $d$ )

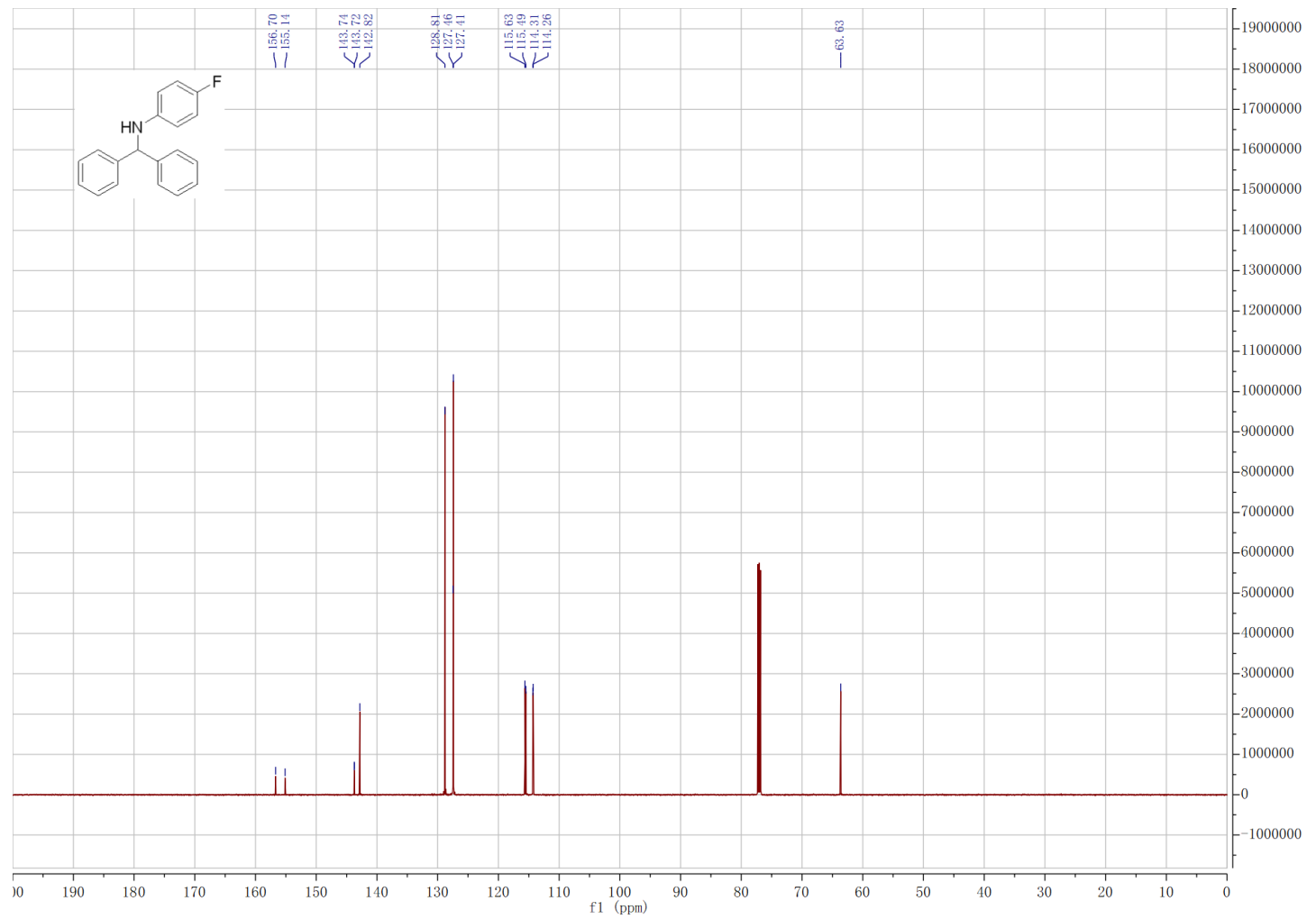


${ }^{19}$ F NMR (564 MHz, Chloroform- $d$ )

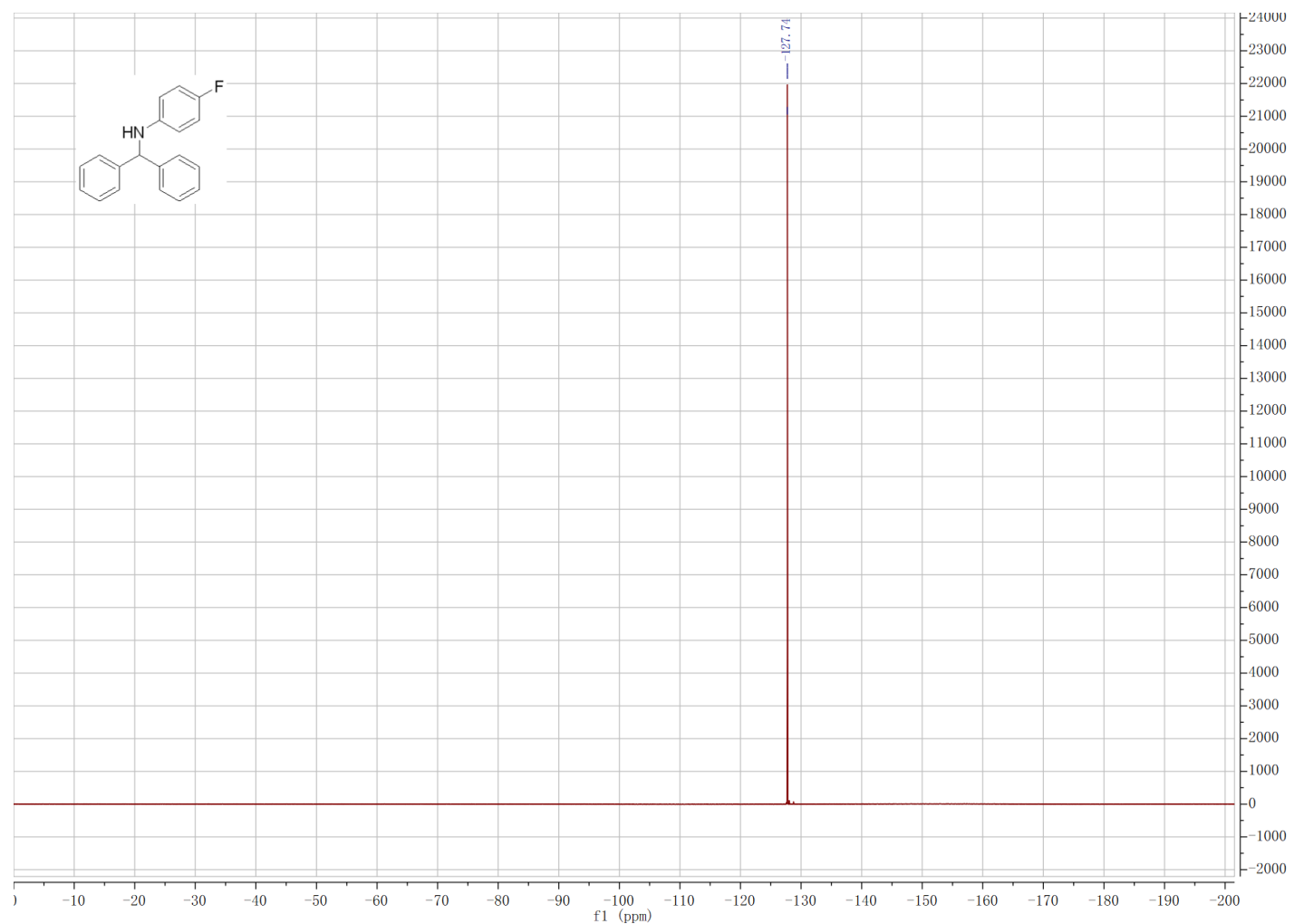


$N$-Benzhydryl-4-chloroaniline (10e)

${ }^{1} \mathrm{H}$ NMR (600 MHz, Chloroform- $d$ )

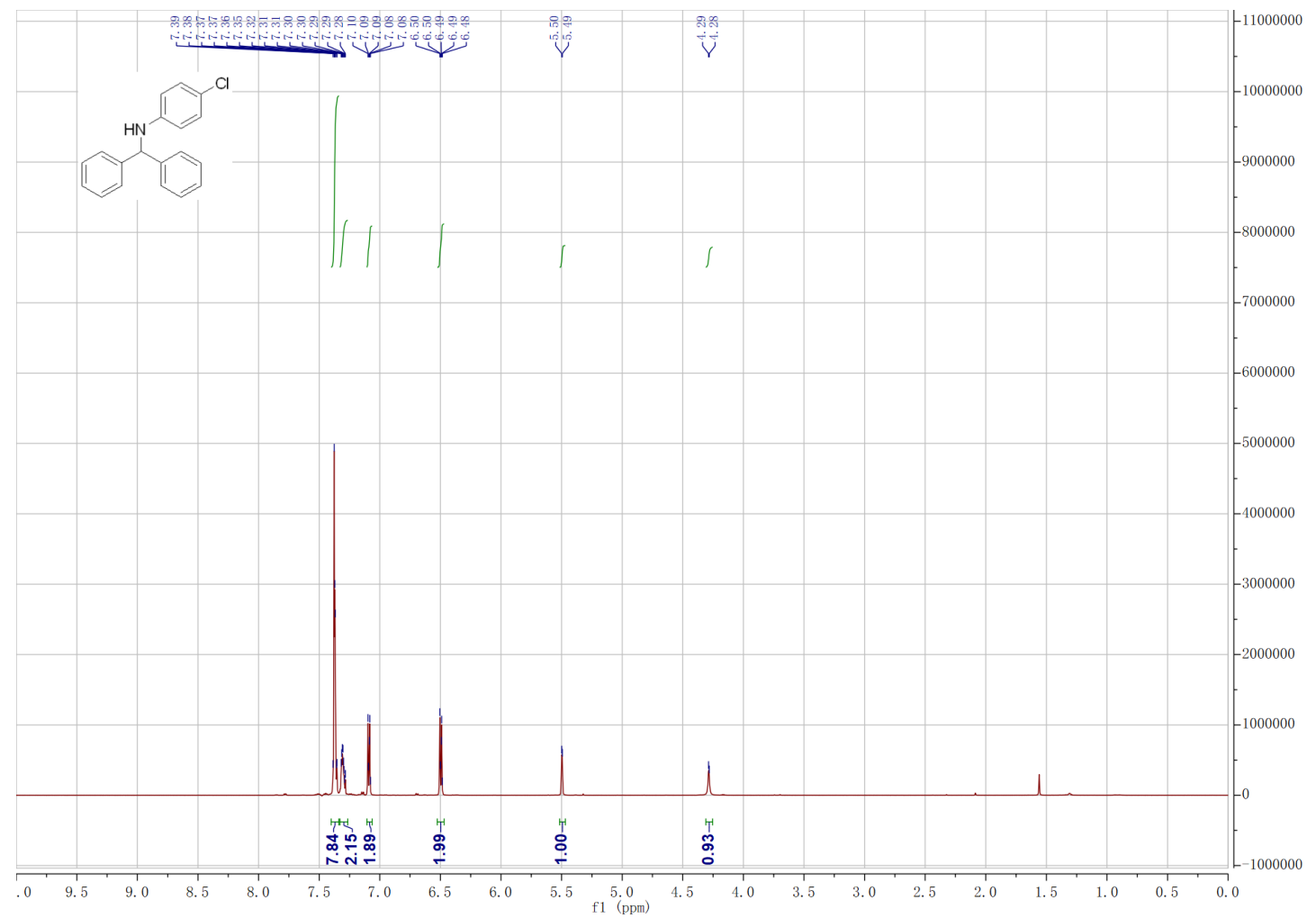

${ }^{13} \mathrm{C}$ NMR (151 MHz, Chloroform- $d$ )

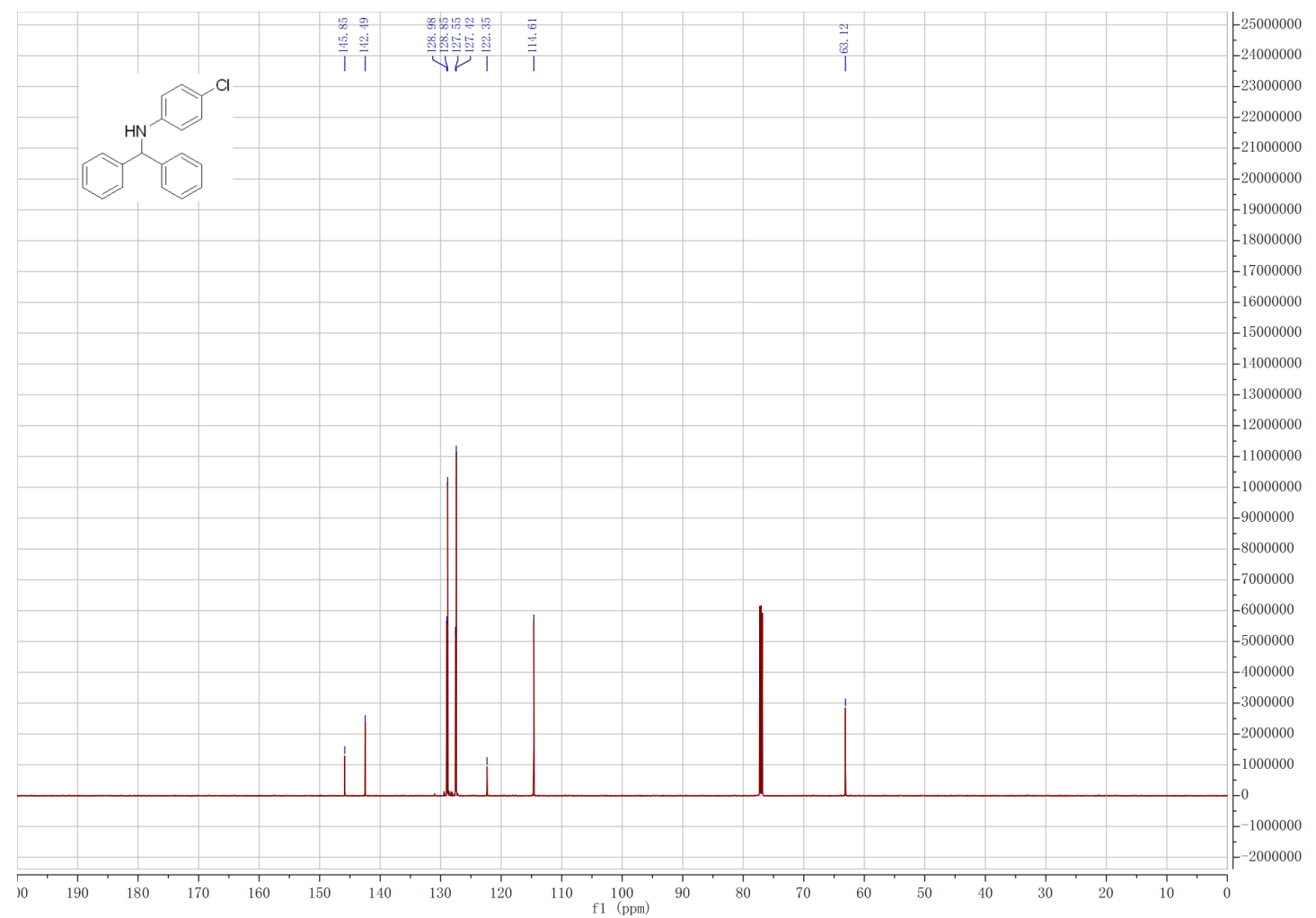


$\mathrm{N}$-Benzhydryl-4-bromoaniline (10f)

${ }^{1} \mathrm{H}$ NMR (600 MHz, Chloroform- $d$ )

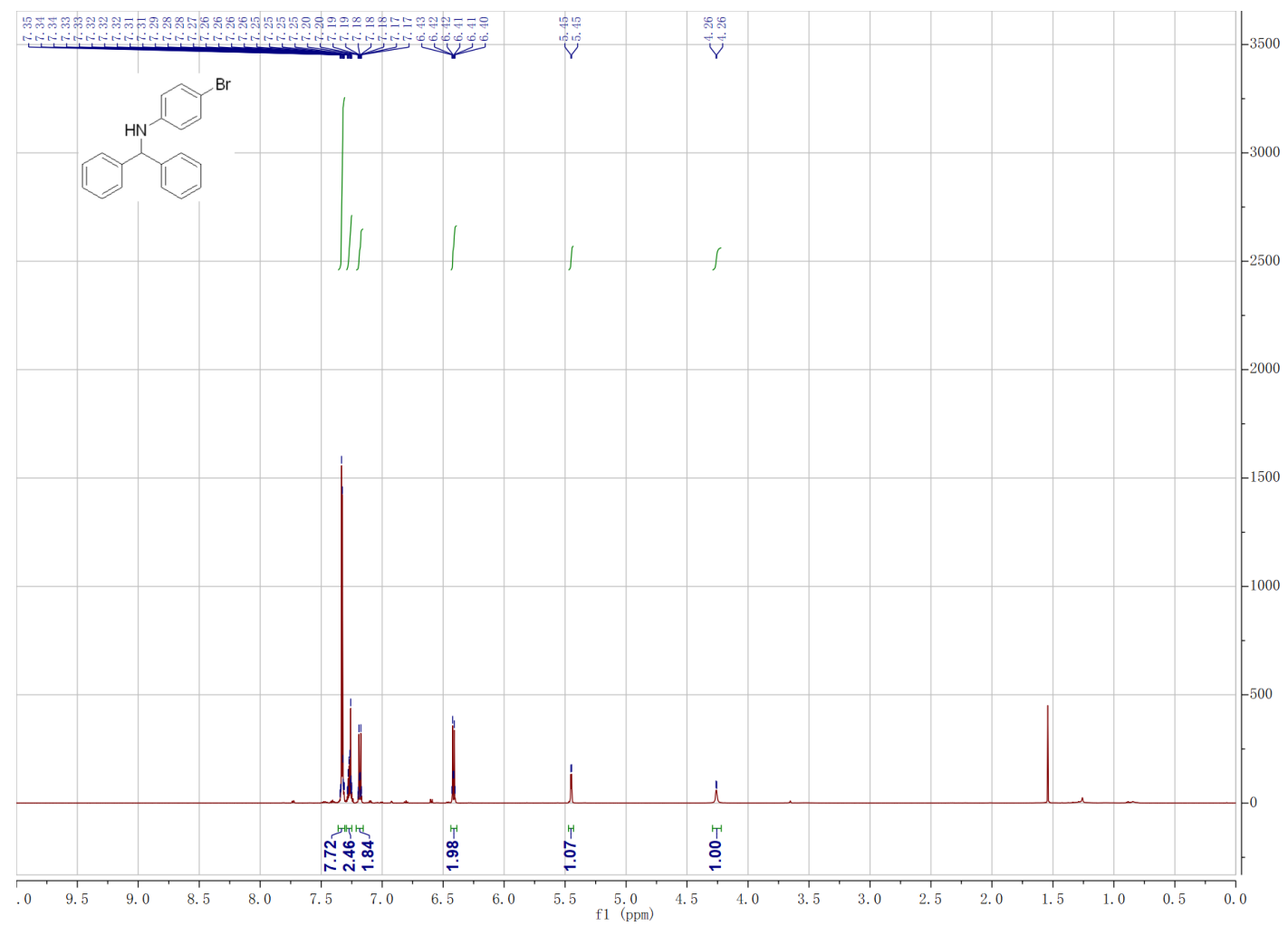

${ }^{13} \mathrm{C}$ NMR (151 MHz, Chloroform- $d$ )

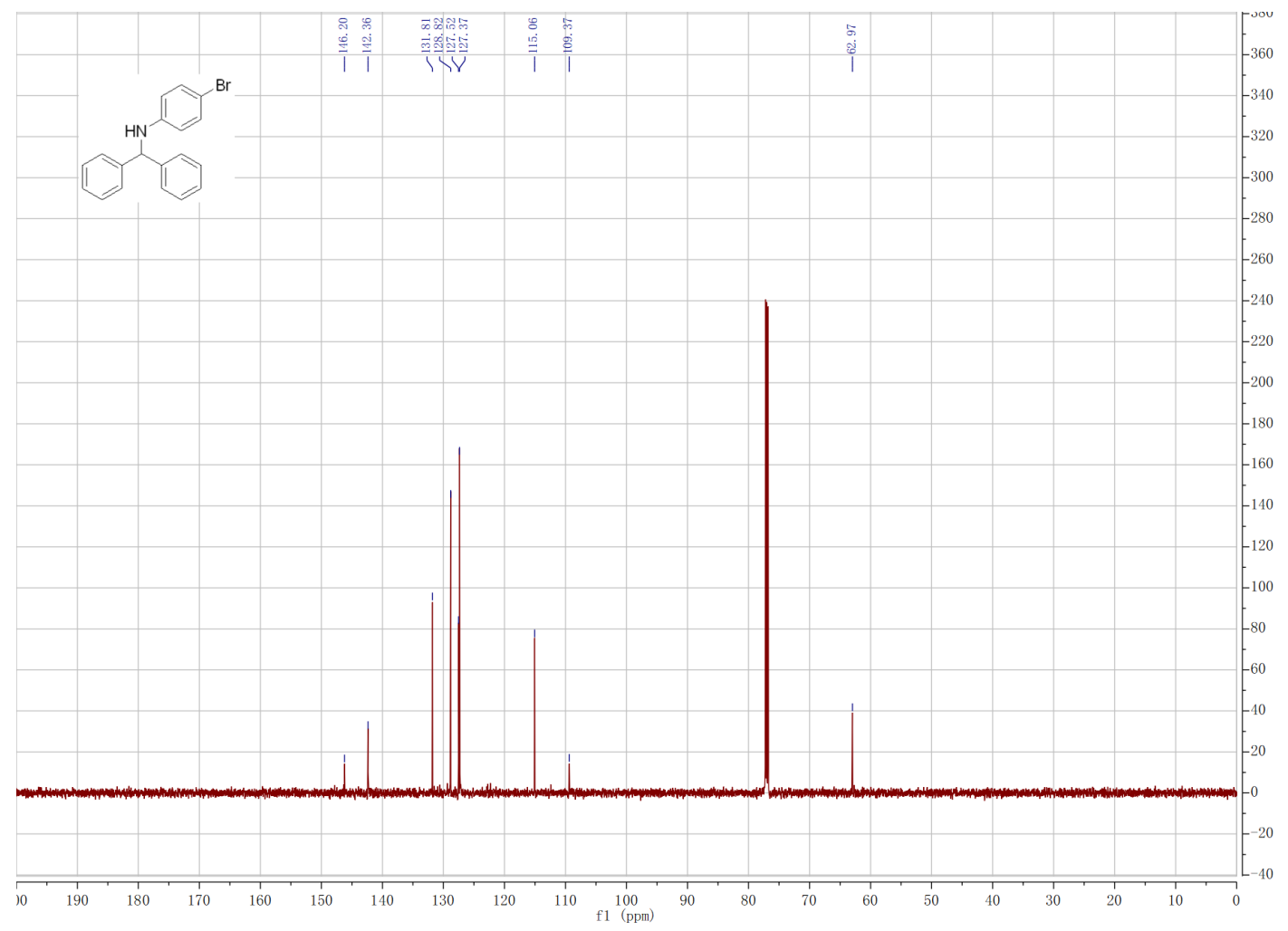


4-(Benzhydrylamino)benzonitrile (10g)

${ }^{1} \mathrm{H}$ NMR (600 MHz, Chloroform- $d$ )

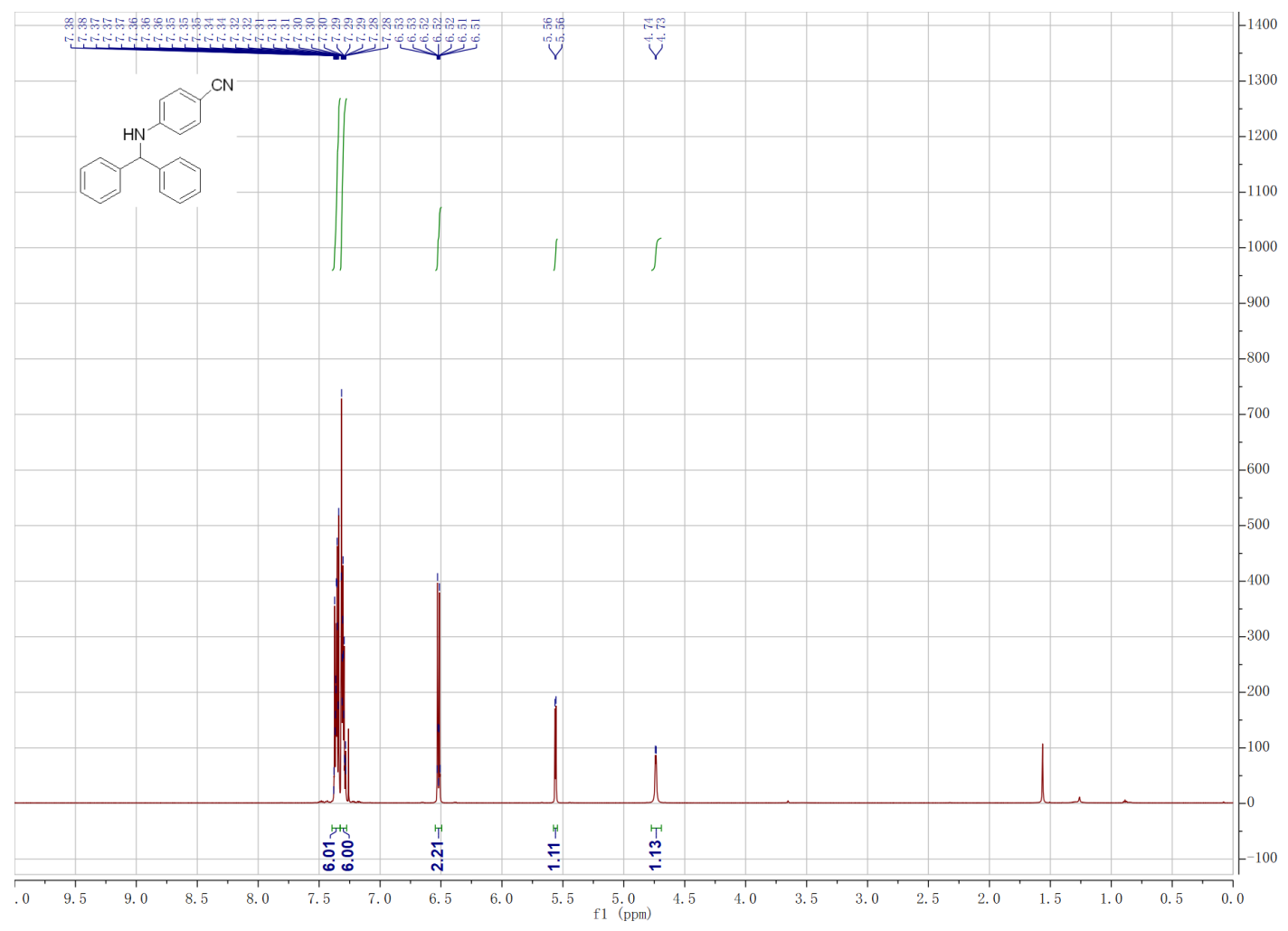

${ }^{13} \mathrm{C}$ NMR (151 MHz, Chloroform- $d$ )
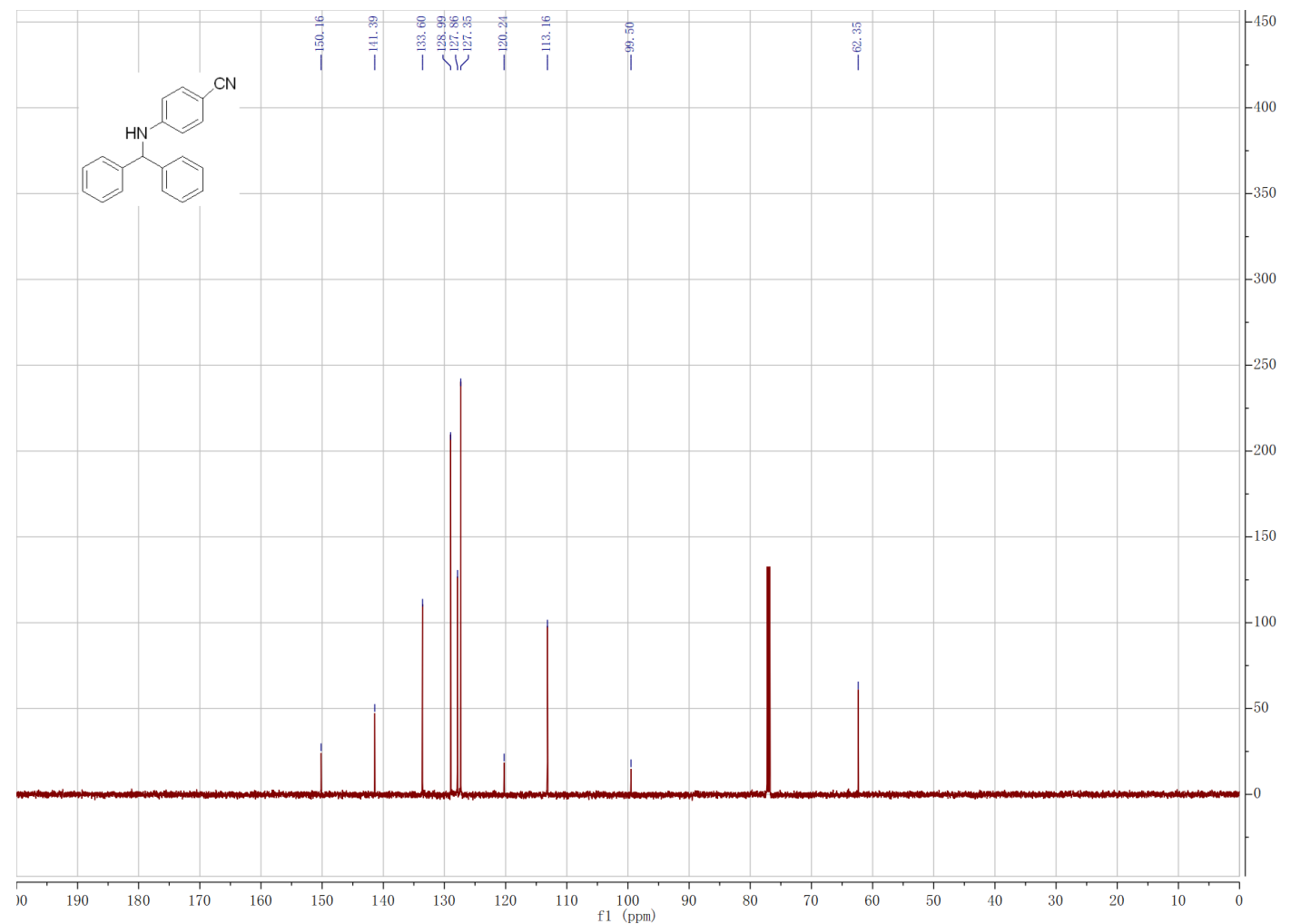
$\mathrm{N}$-Benzhydryl-3-methoxyaniline (10h)

${ }^{1} \mathrm{H}$ NMR (600 MHz, Chloroform-d)

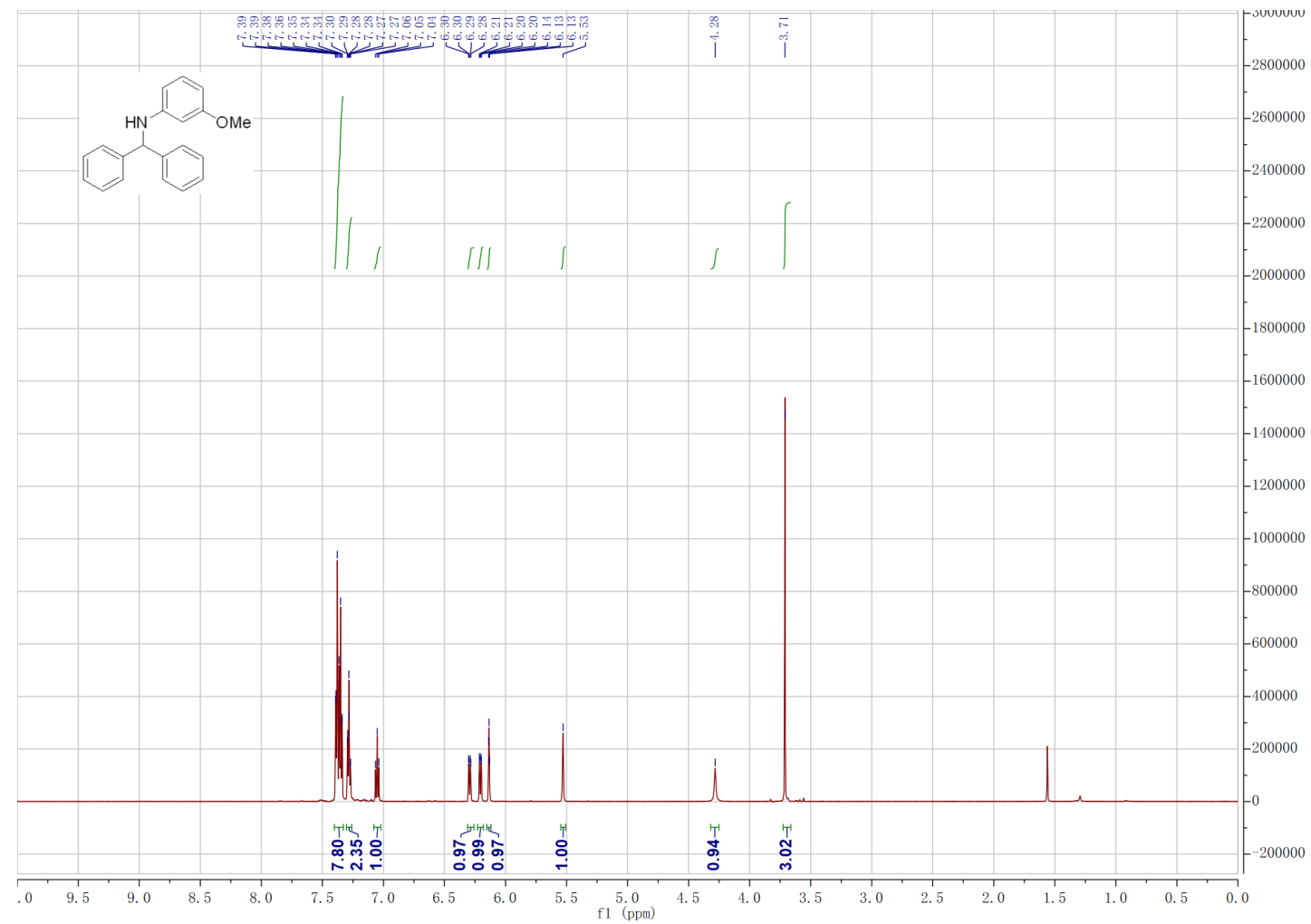

${ }^{13} \mathrm{C}$ NMR (151 MHz, Chloroform- $d$ )

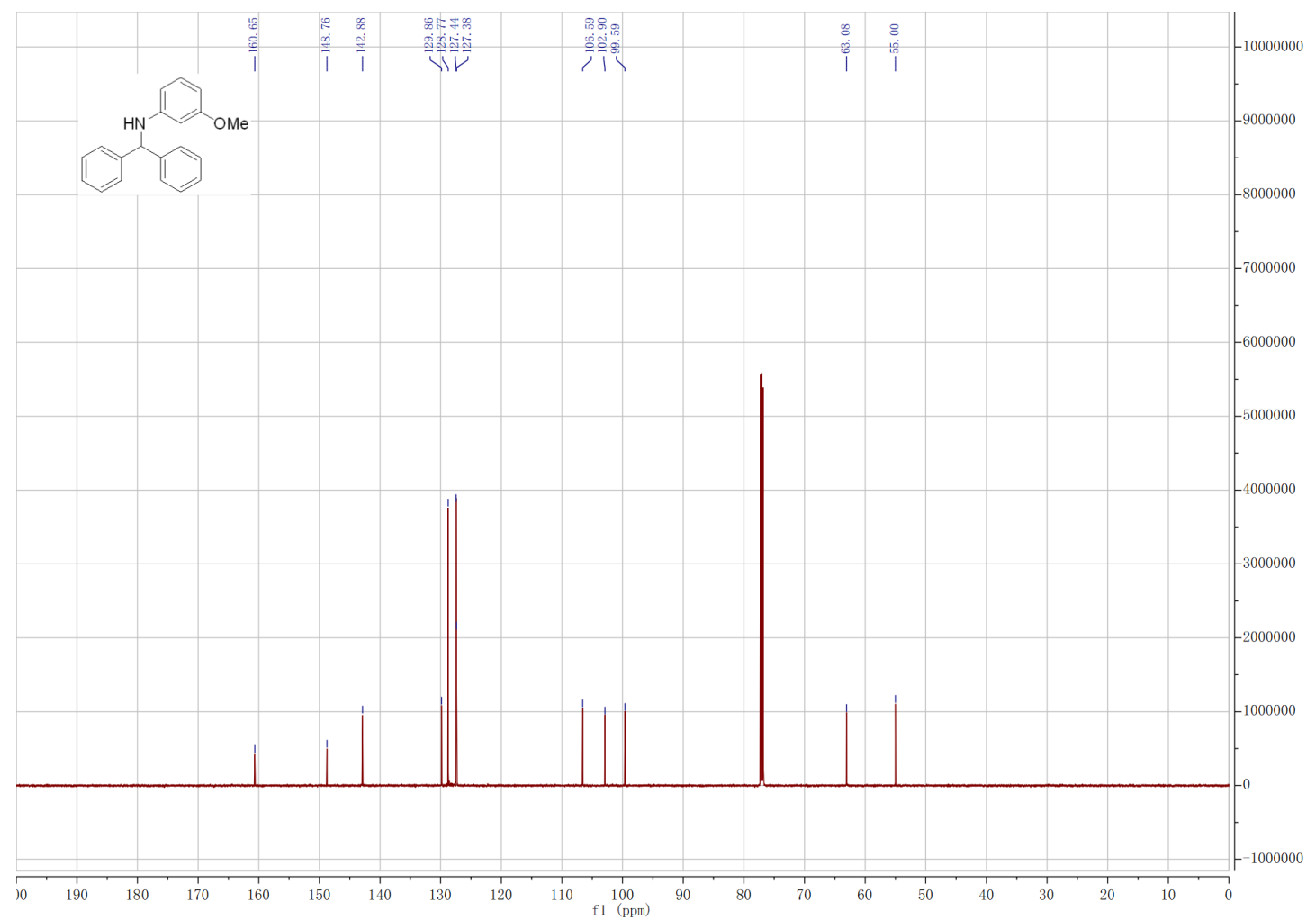


$N$-Benzhydryl-3-bromoaniline (10i)

${ }^{1} \mathrm{H}$ NMR (600 MHz, Chloroform- $d$ )

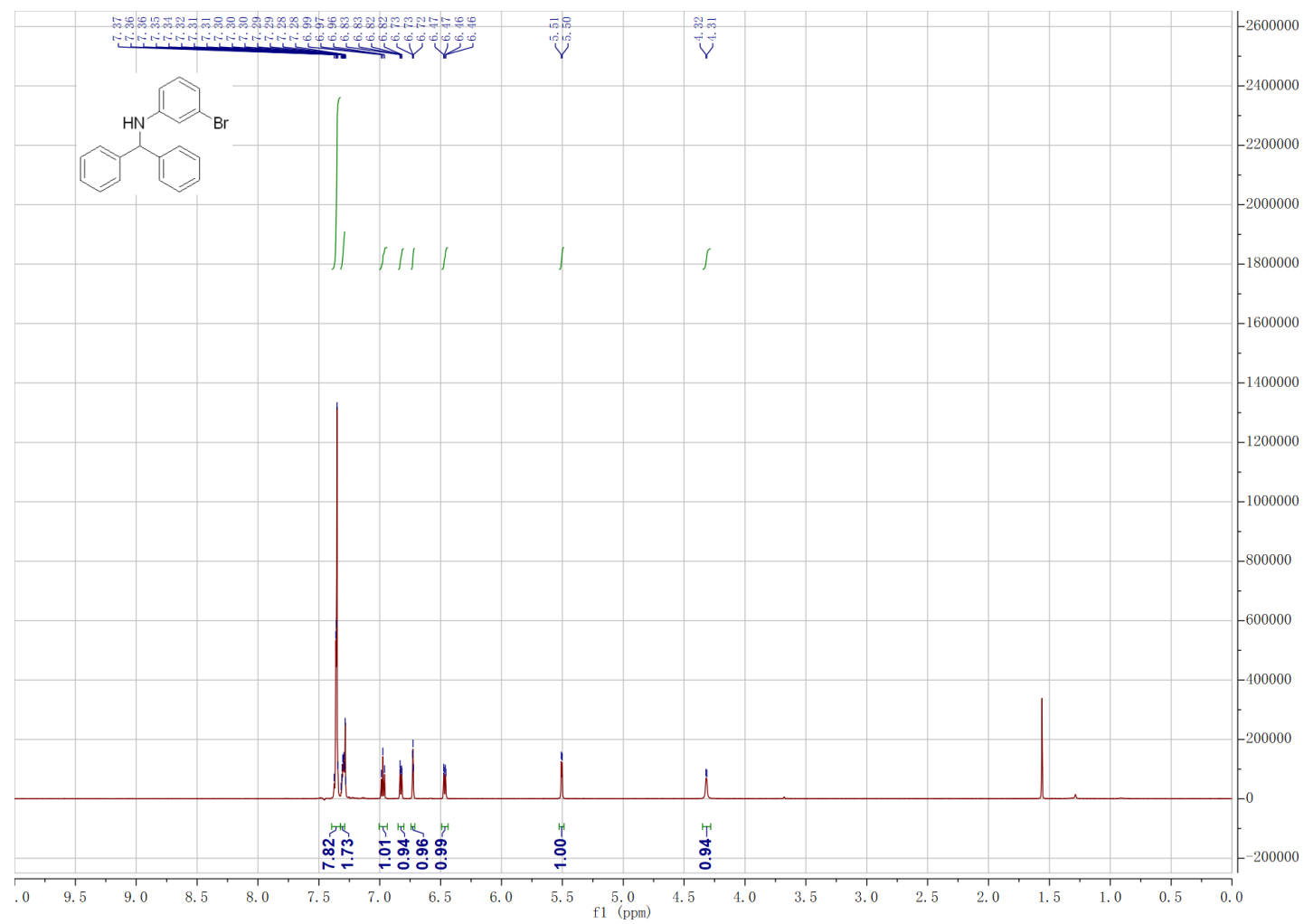

${ }^{13} \mathrm{C}$ NMR (151 MHz, Chloroform-d)

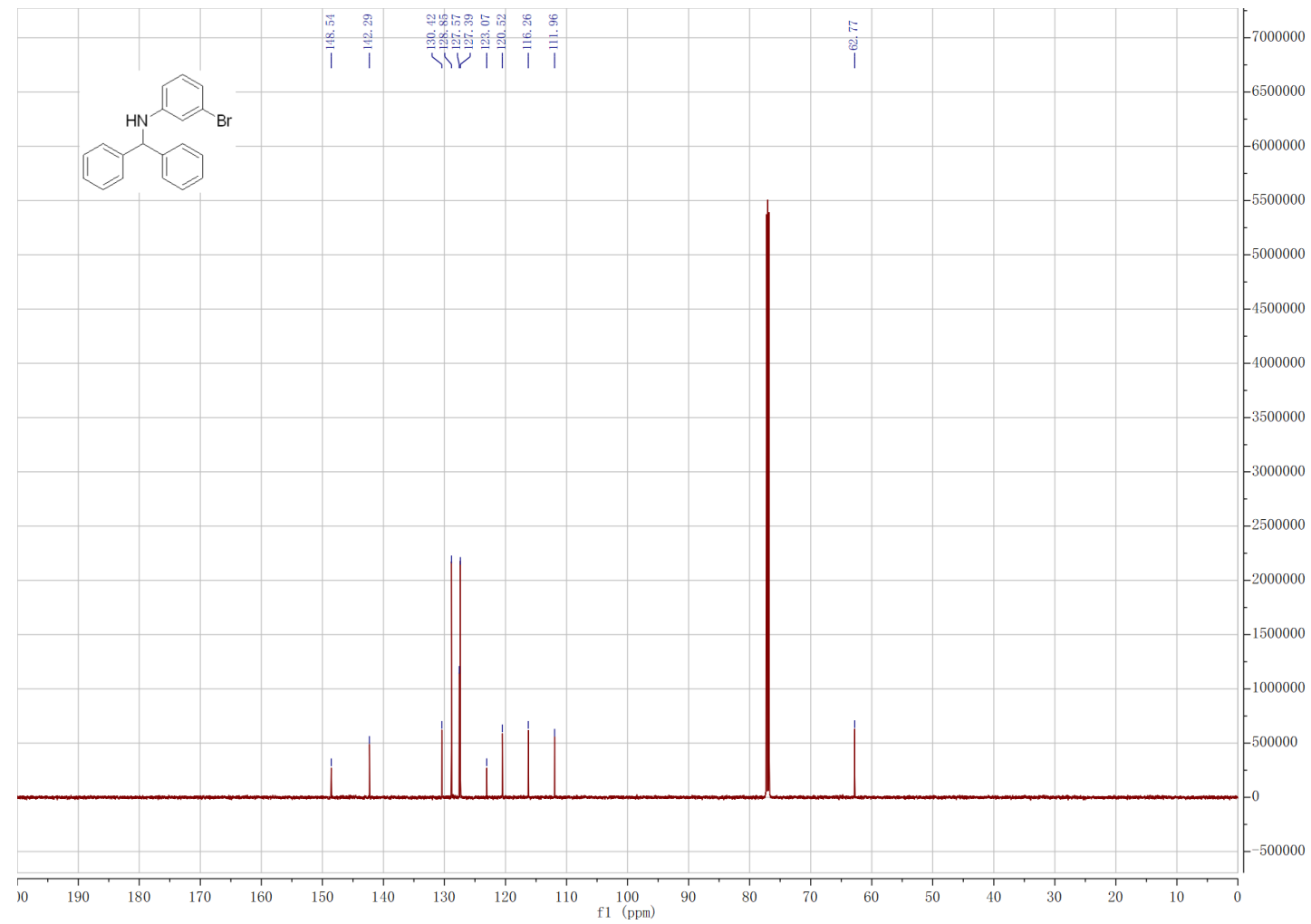


$N$-Benzhydryl-3-iodoaniline (10j)

${ }^{1} \mathrm{H}$ NMR (600 MHz, Chloroform- $d$ )

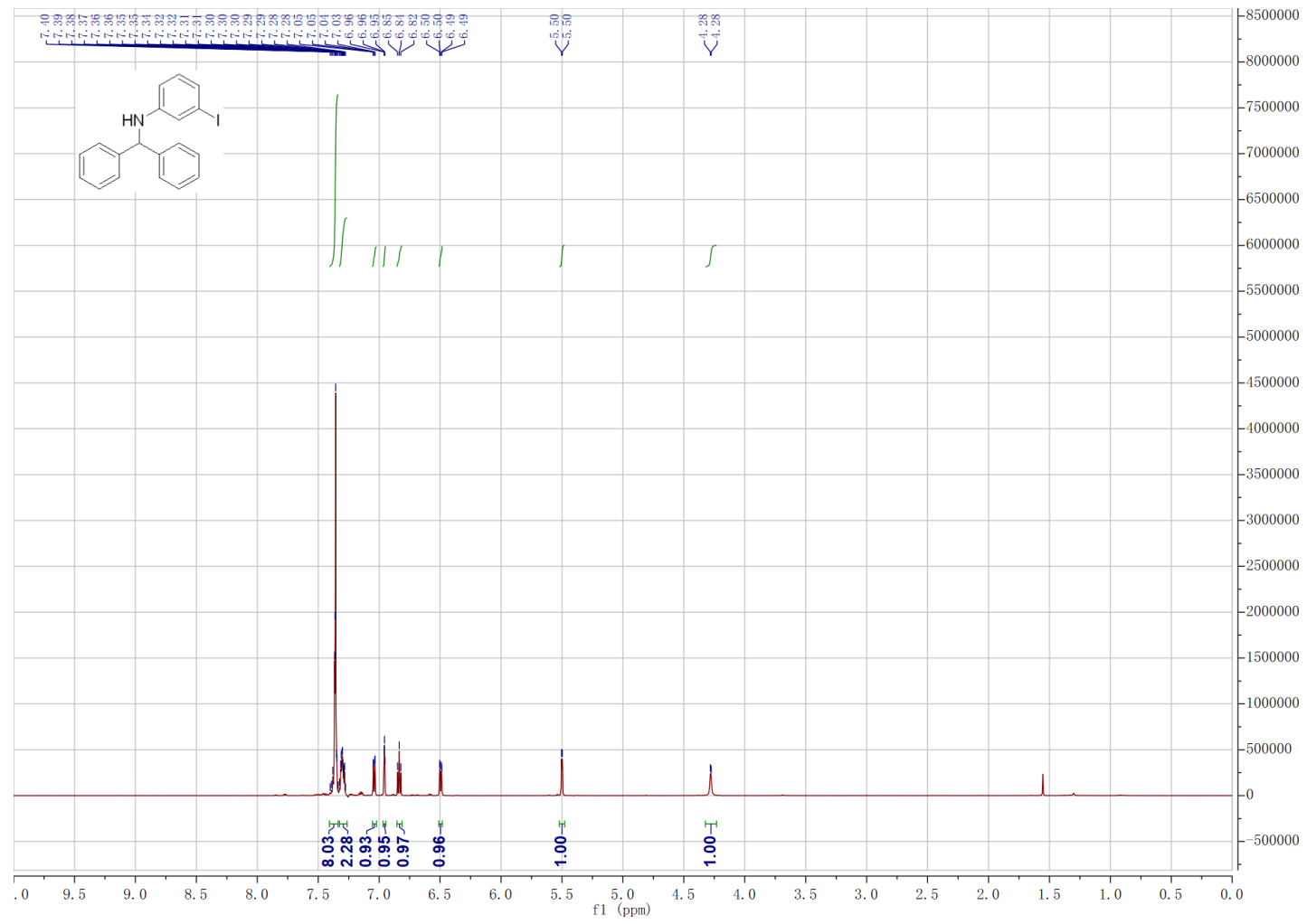

${ }^{13} \mathrm{C}$ NMR (151 MHz, Chloroform- $d$ )

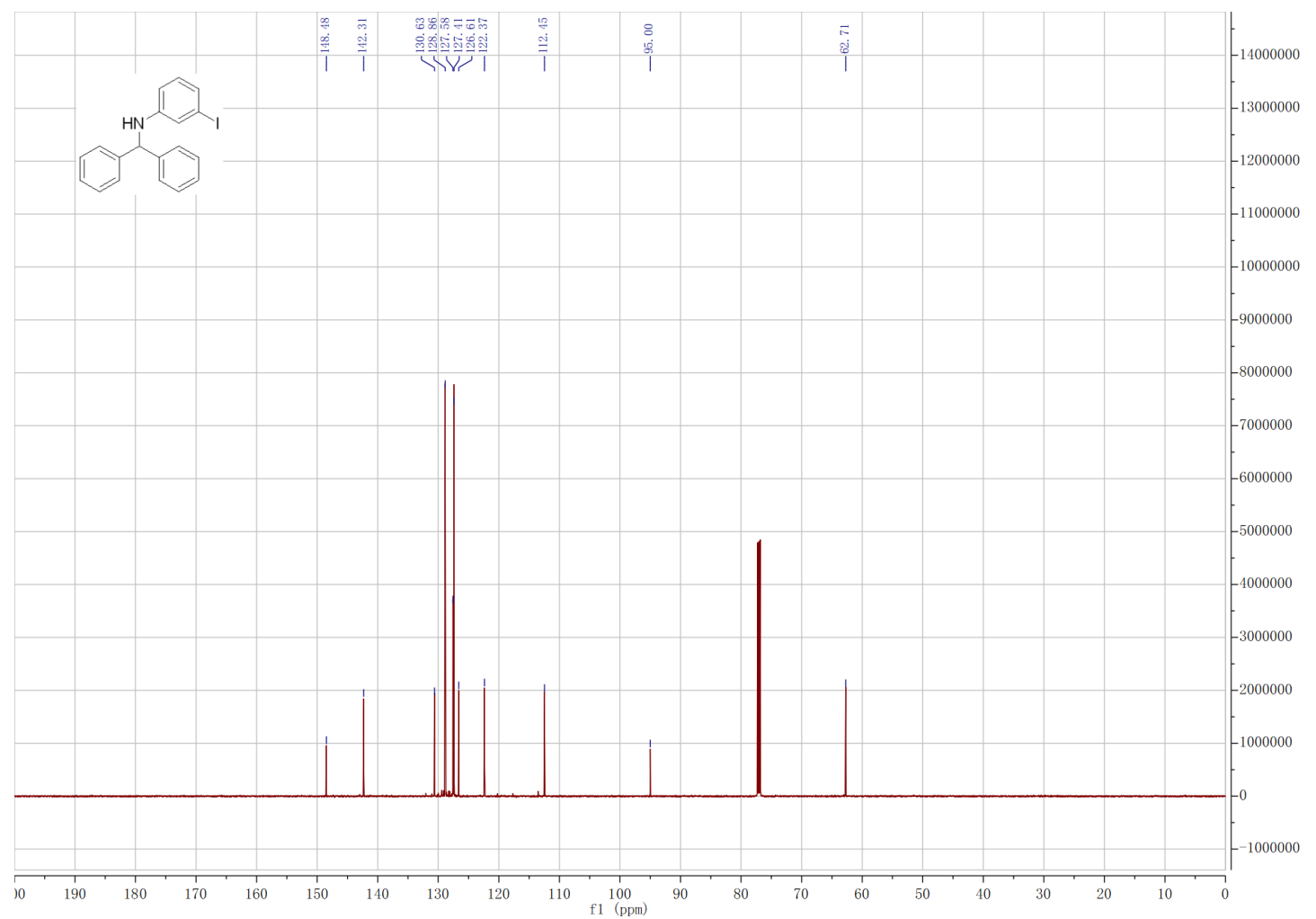


$\mathrm{N}$-Benzhydryl-2-chloroaniline (10k)

${ }^{1} \mathrm{H}$ NMR (600 MHz, Chloroform- $d$ )

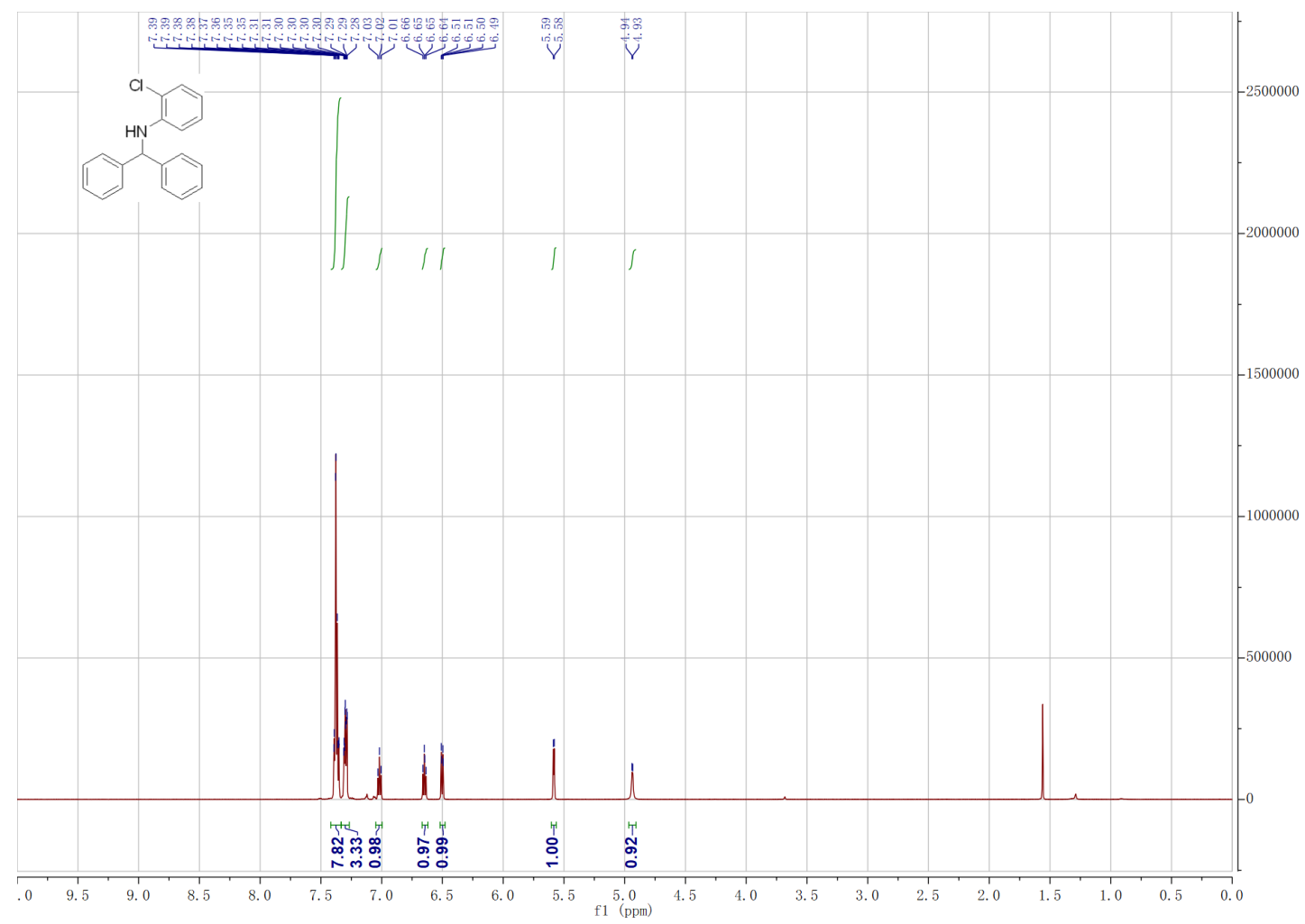

${ }^{13} \mathrm{C}$ NMR (151 MHz, Chloroform- $d$ )

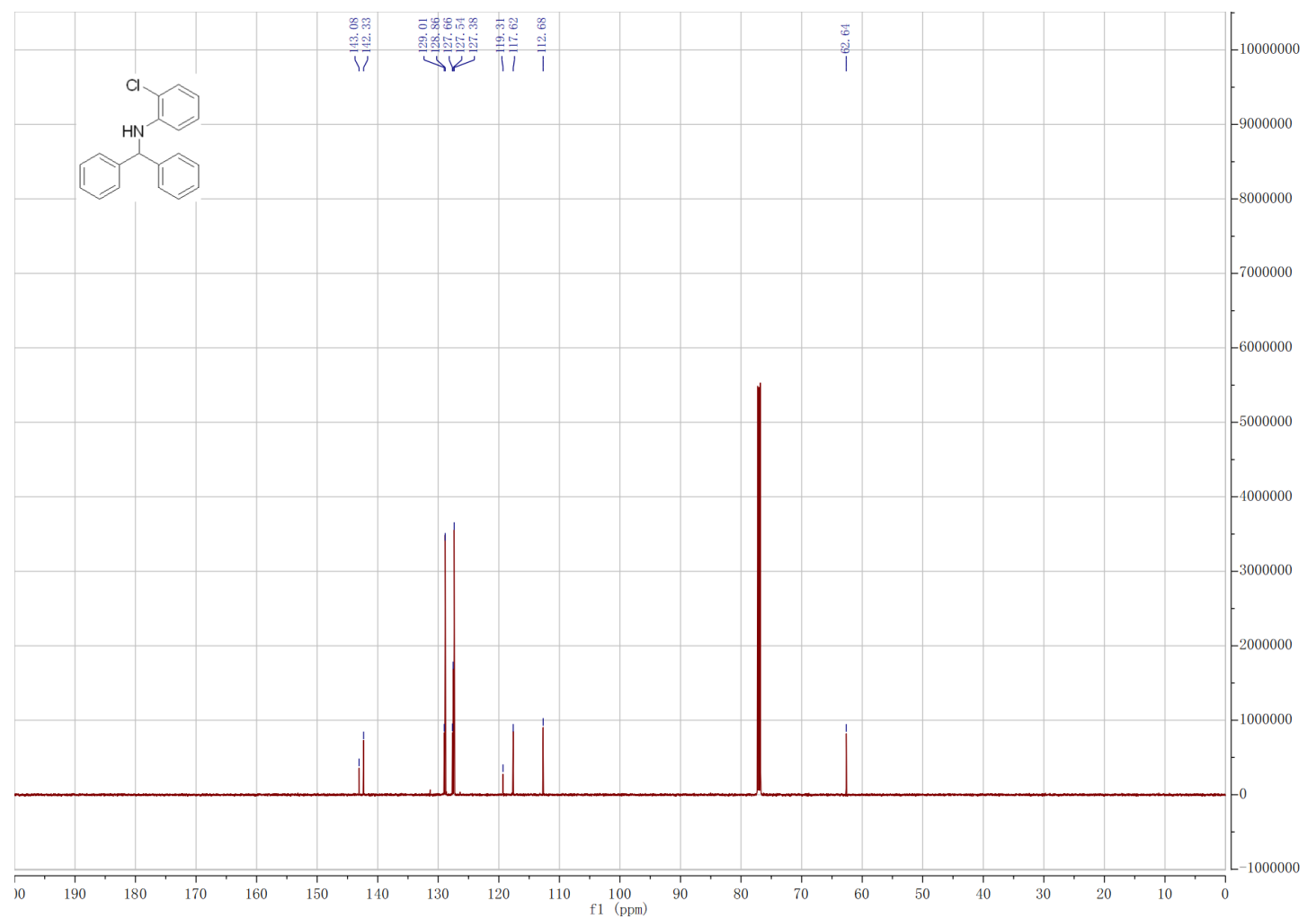


$N$-Benzhydryl-2-bromoaniline (101)

${ }^{1} \mathrm{H}$ NMR (600 MHz, Chloroform- $d$ )

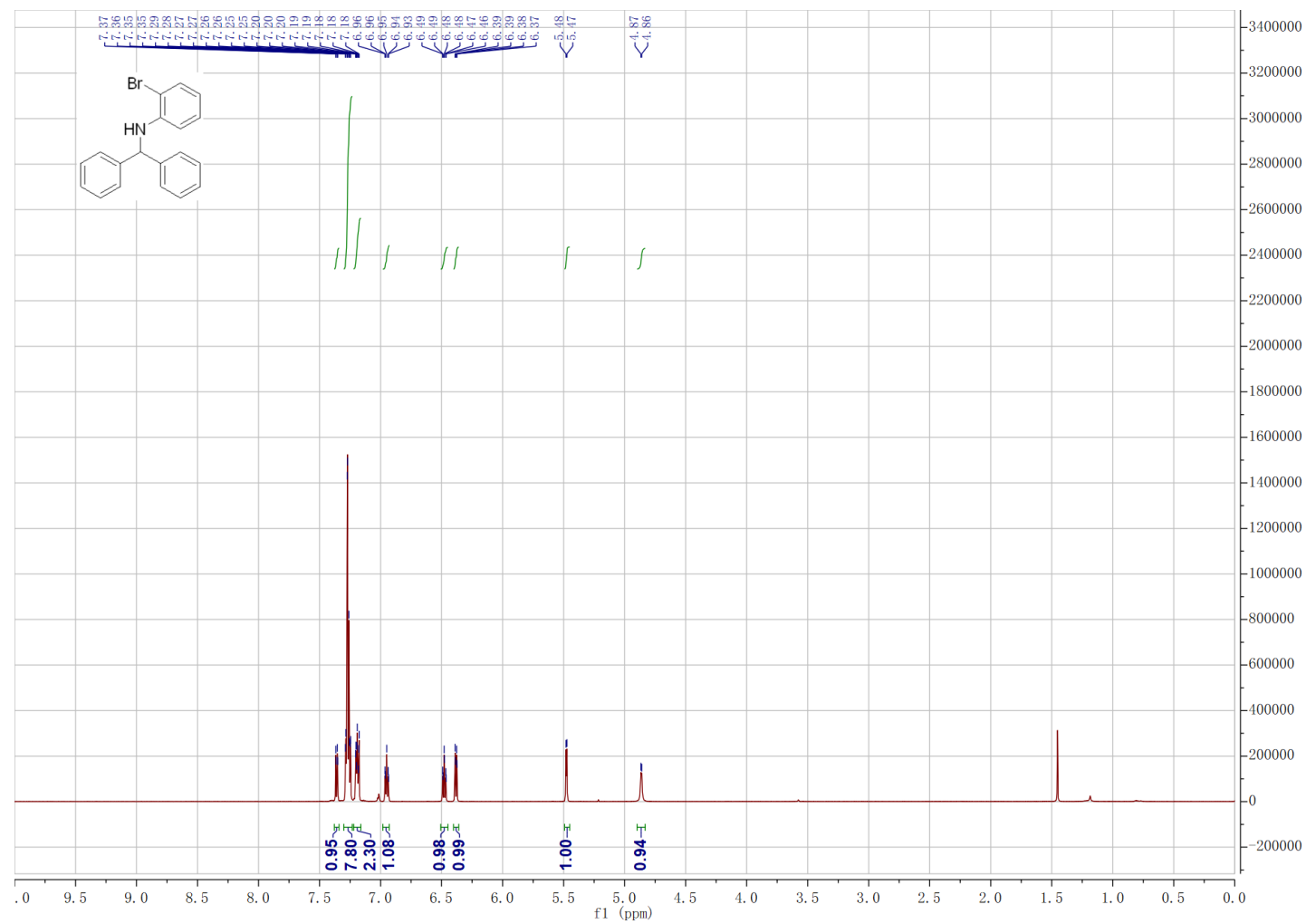

${ }^{13} \mathrm{C}$ NMR (151 MHz, Chloroform- $d$ )

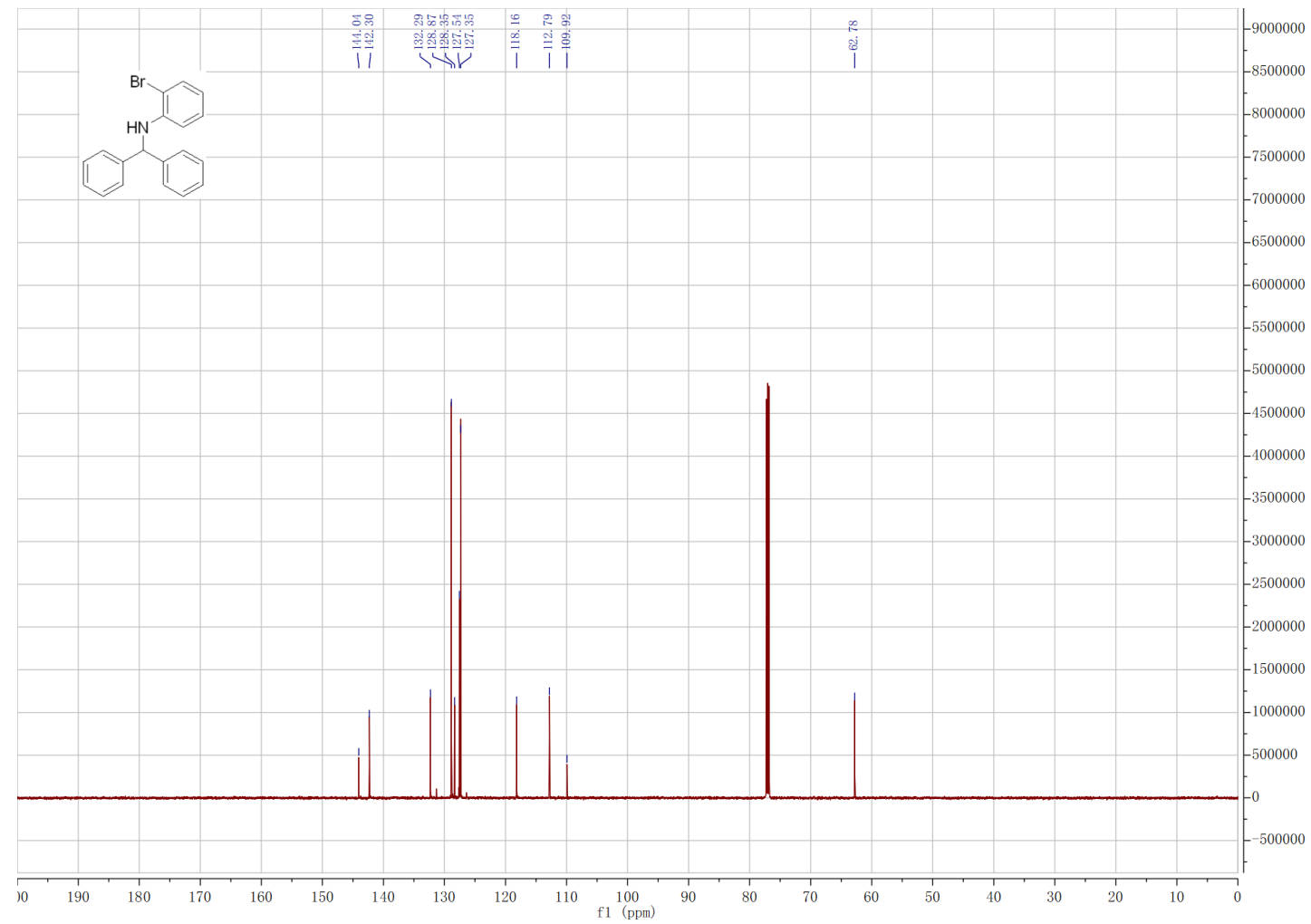


$\mathrm{N}$-Benzhydryl-2-methylaniline (10m)

${ }^{1} \mathrm{H}$ NMR (600 MHz, Chloroform- $d$ )

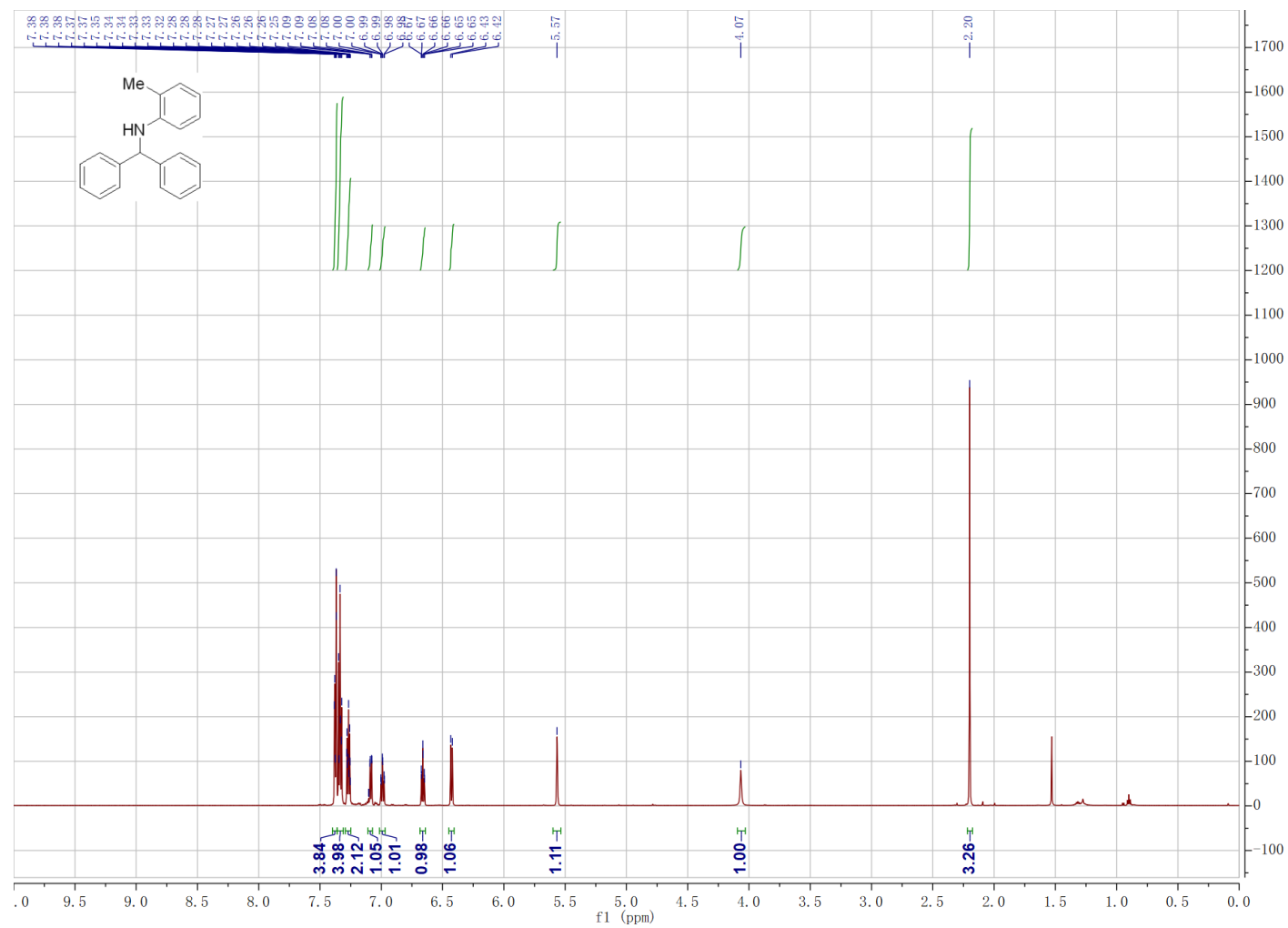

${ }^{13} \mathrm{C}$ NMR (151 MHz, Chloroform- $d$ )
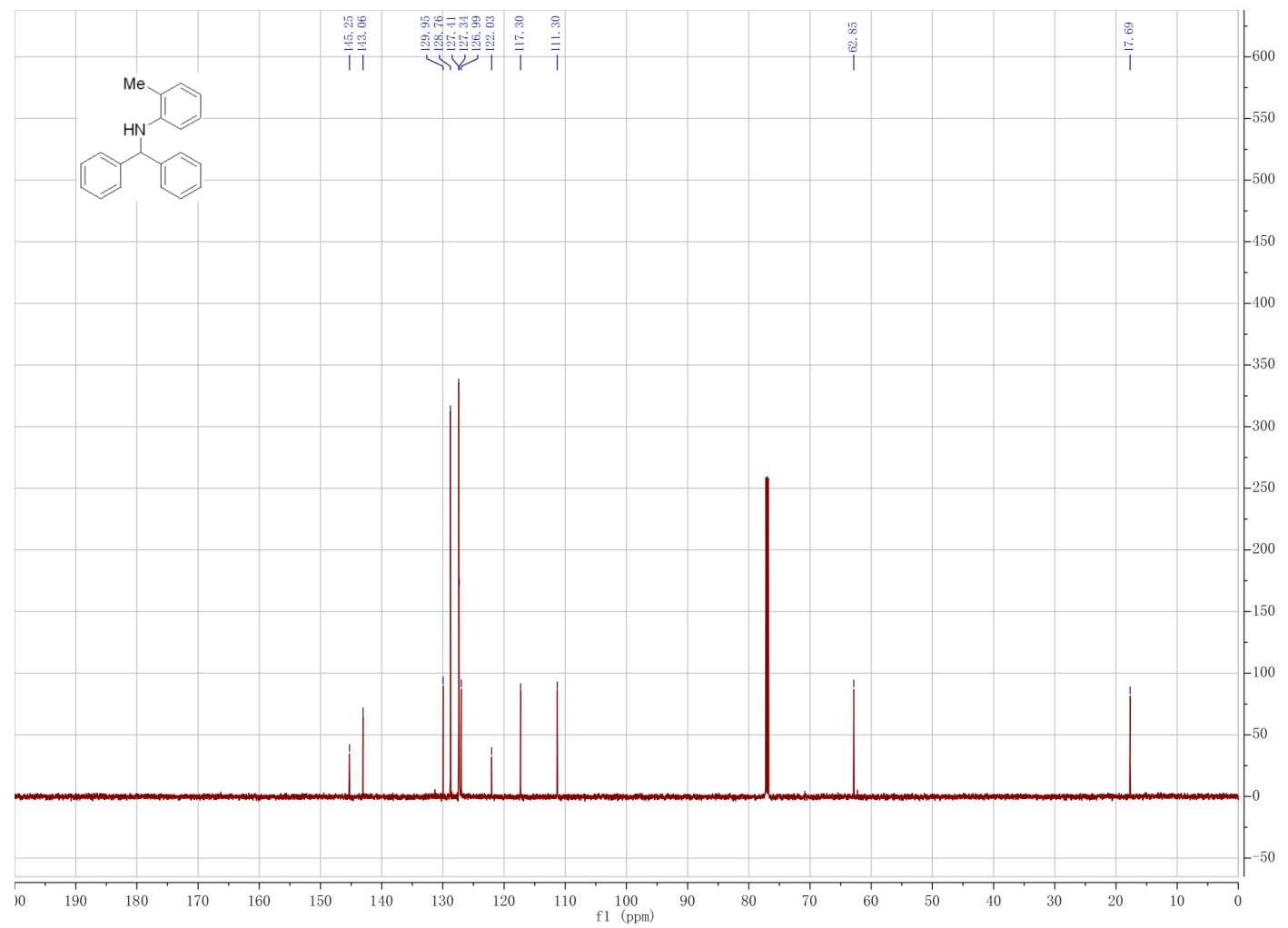
N-Benzhydryl-2-methoxyaniline (10n)

${ }^{1} \mathrm{H}$ NMR (600 MHz, Chloroform- $d$ )

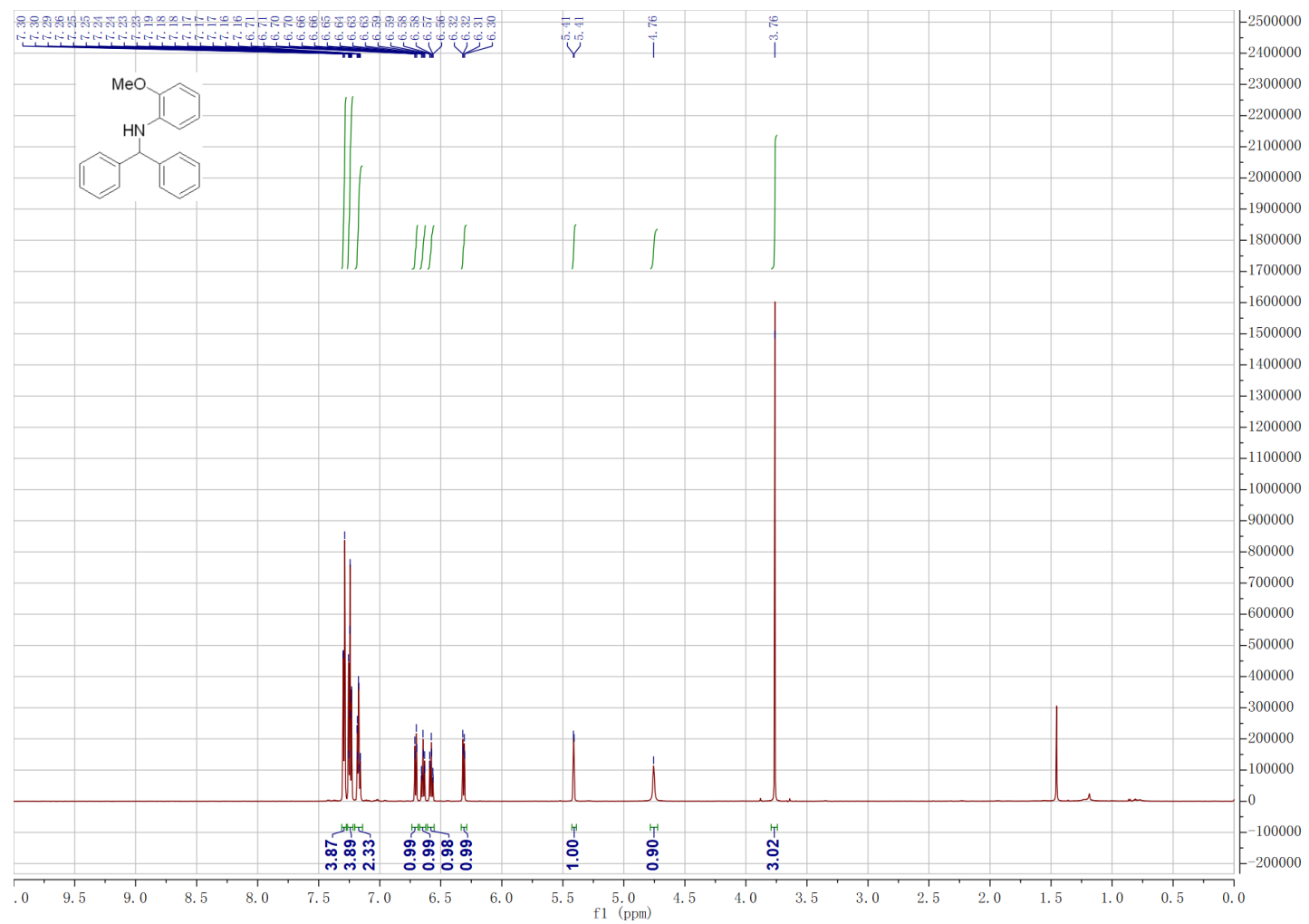

${ }^{13} \mathrm{C}$ NMR (151 MHz, Chloroform- $d$ )

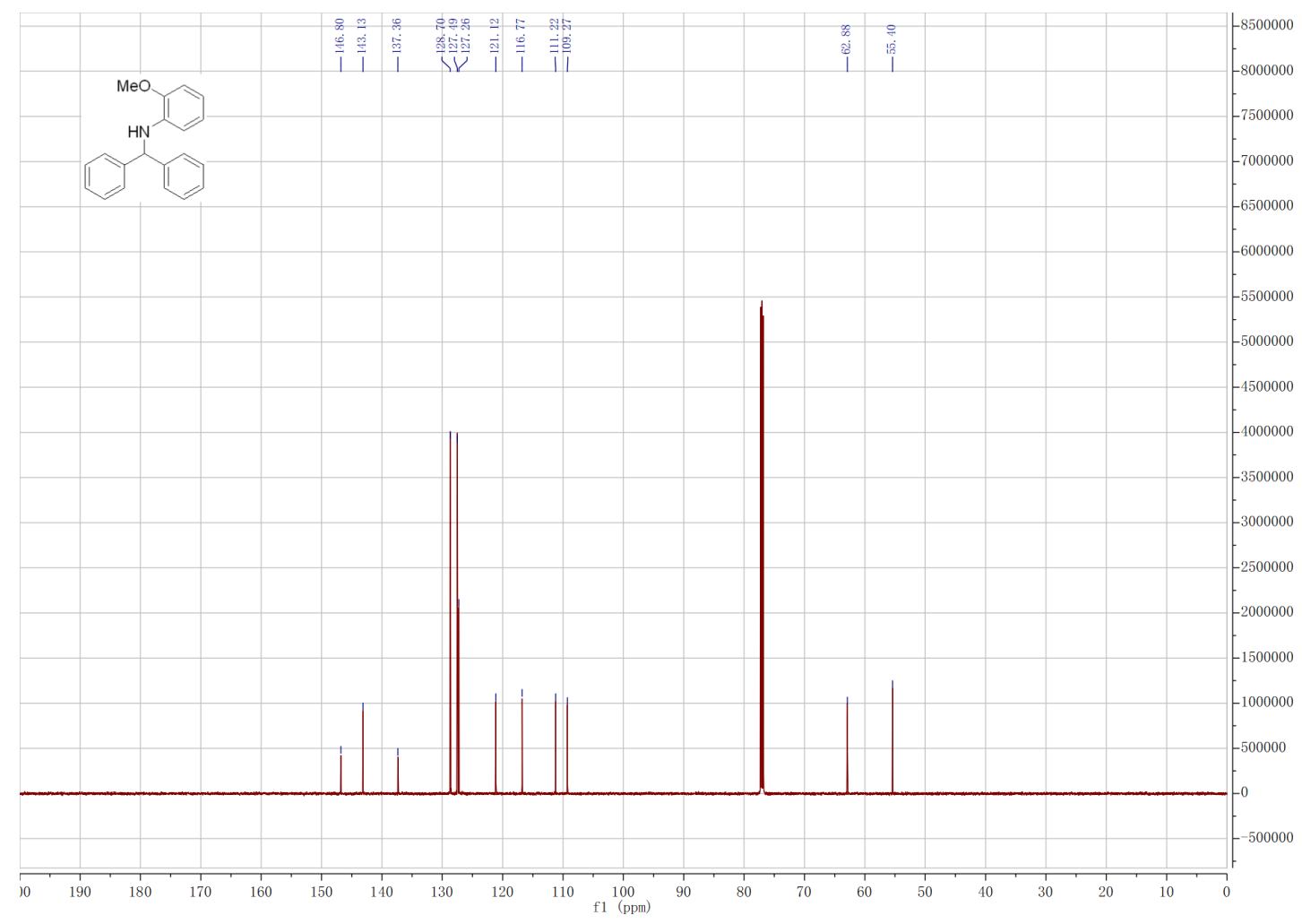


Methyl 2-(benzhydrylamino)benzoate (10o)

${ }^{1} \mathrm{H}$ NMR (600 MHz, Chloroform- $d$ )

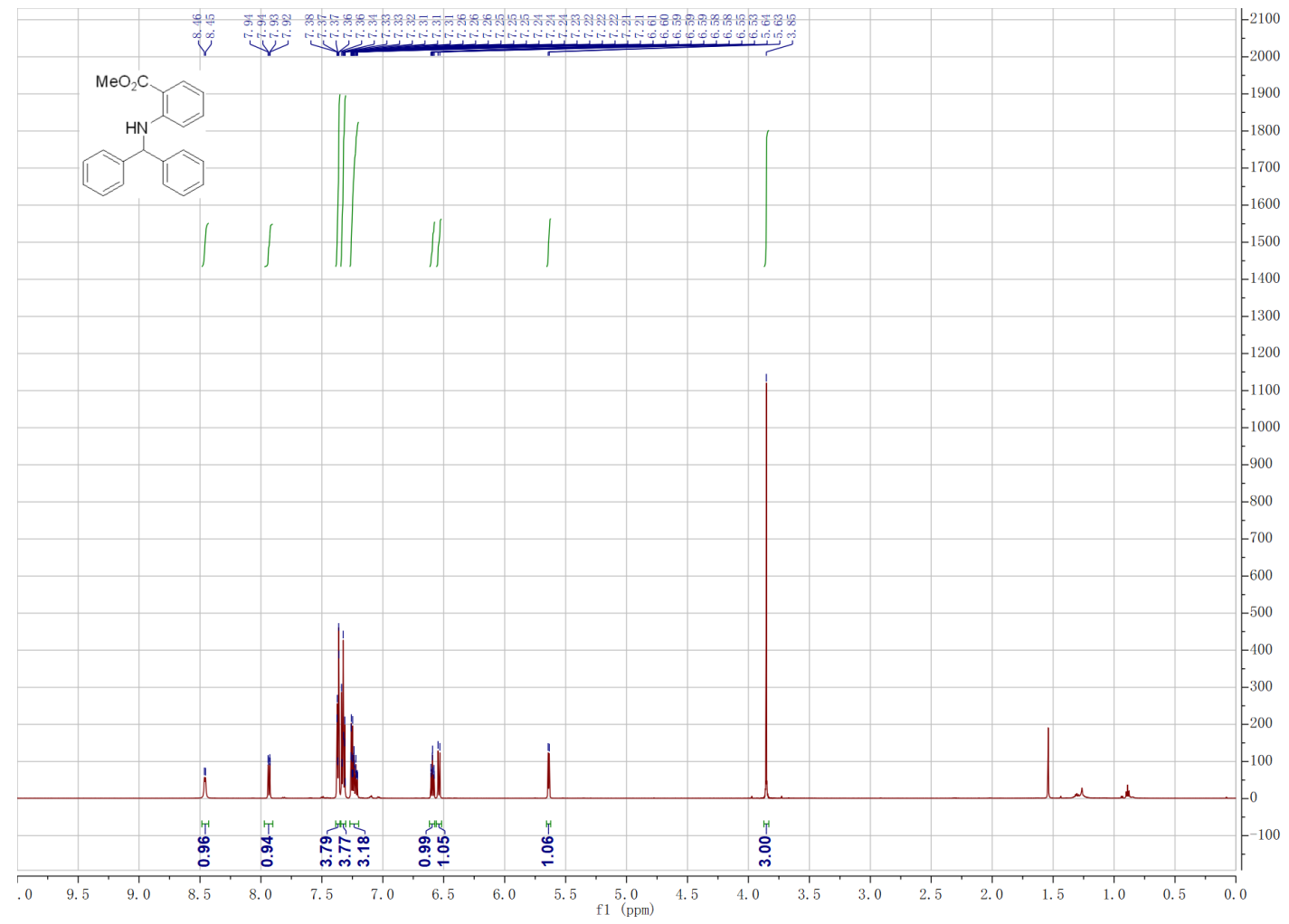

${ }^{13} \mathrm{C}$ NMR (151 MHz, Chloroform- $d$ )

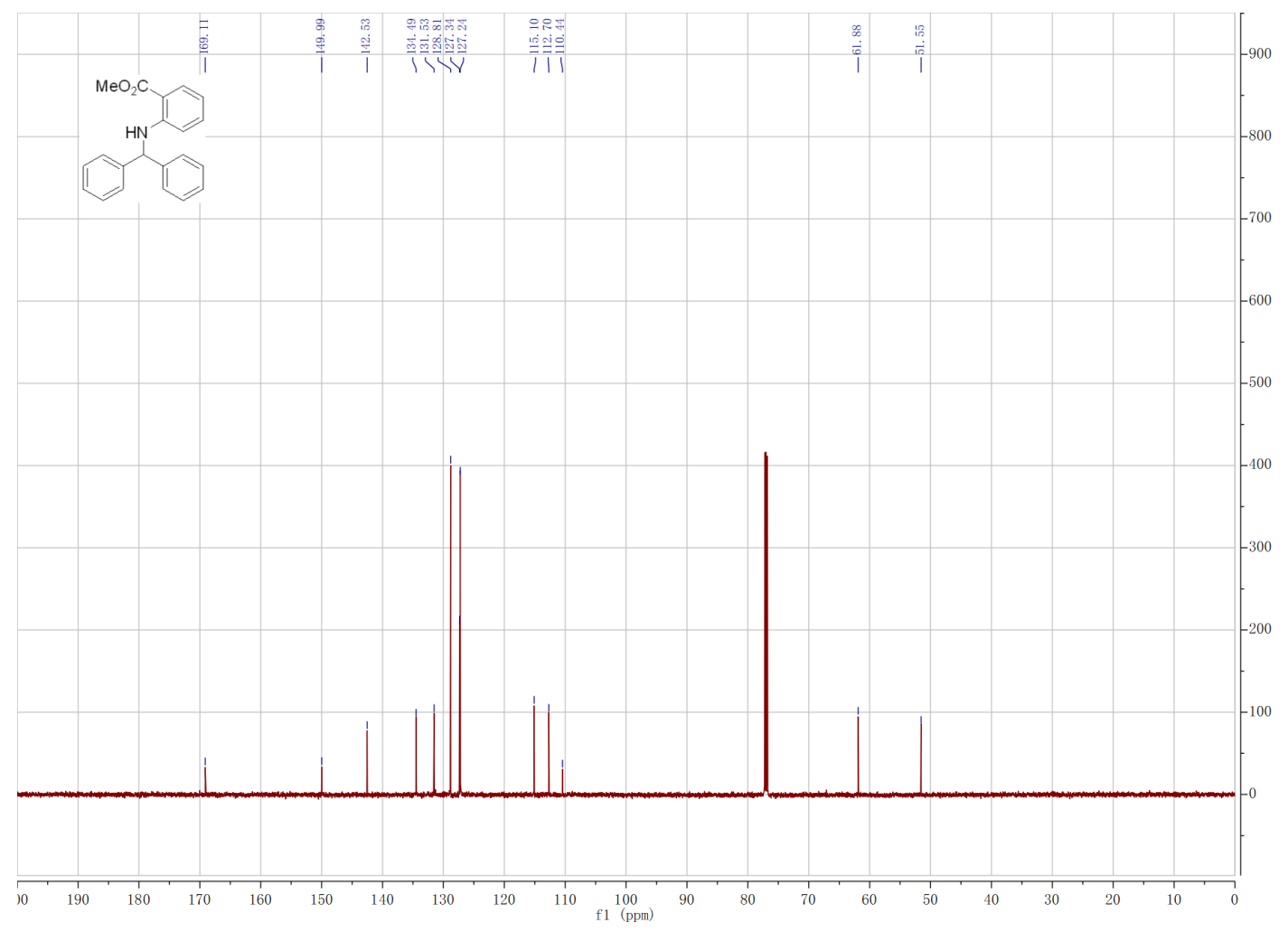


N-Benzhydrylnaphthalen-1-amine (10p)

${ }^{1} \mathrm{H}$ NMR (600 MHz, Chloroform-d)

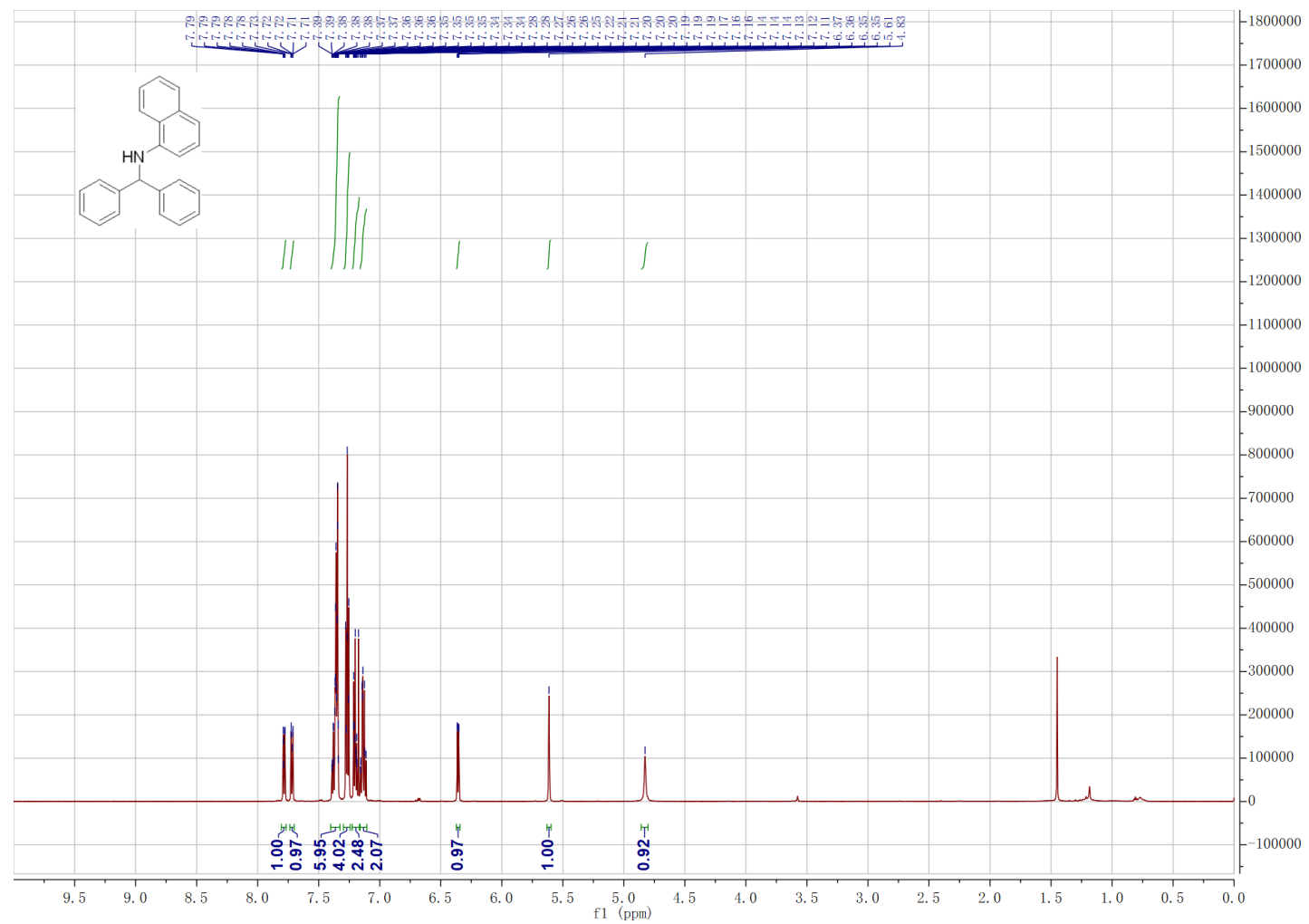

${ }^{13} \mathrm{C}$ NMR (151 MHz, Chloroform- $d$ )

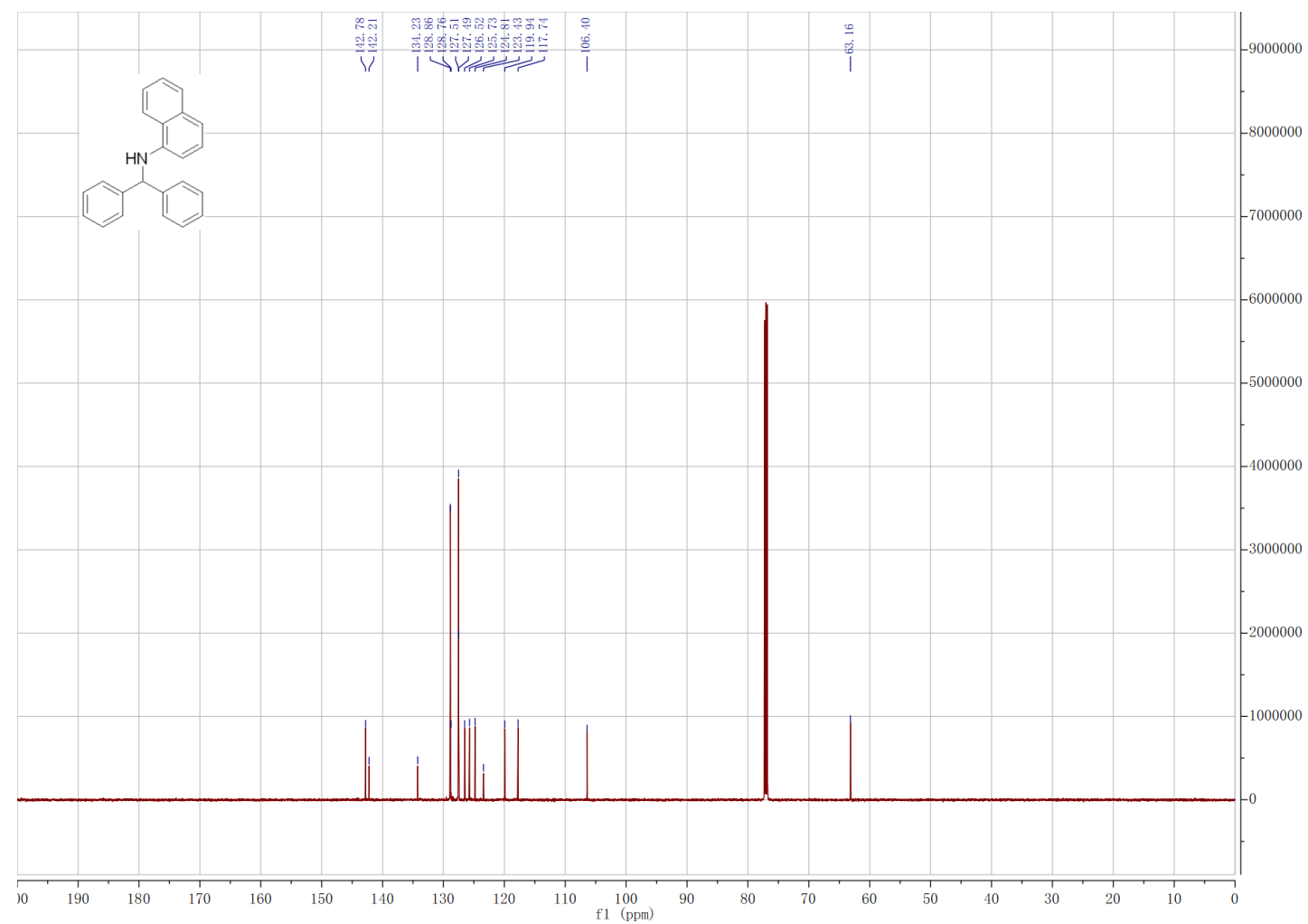


$N$-Benzhydrylbenzo[ $d][1,3]$ dioxol-5-amine (10q)

${ }^{1} \mathrm{H}$ NMR (600 MHz, Chloroform- $d$ )

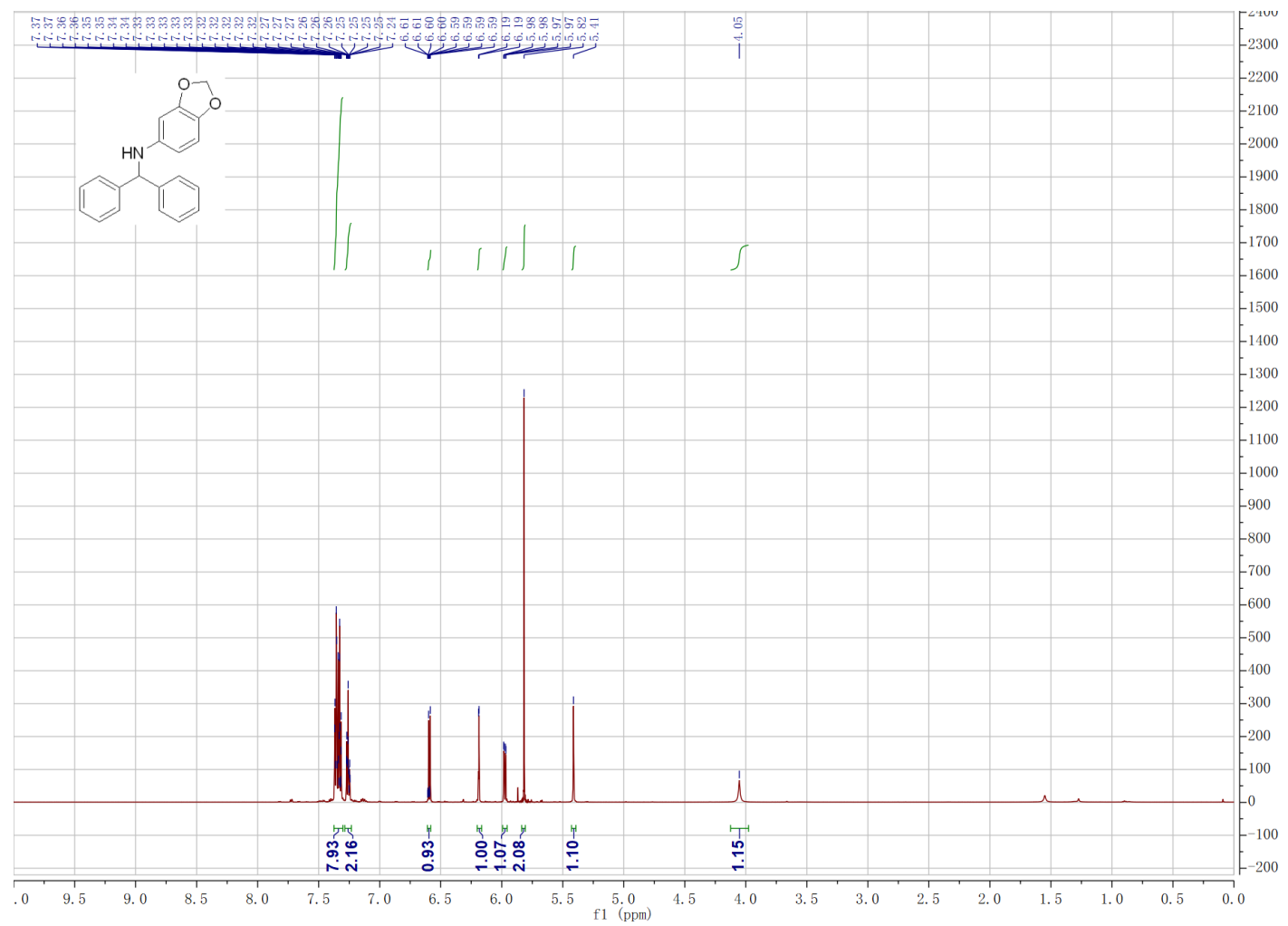

${ }^{13} \mathrm{C}$ NMR (151 MHz, Chloroform- $d$ )

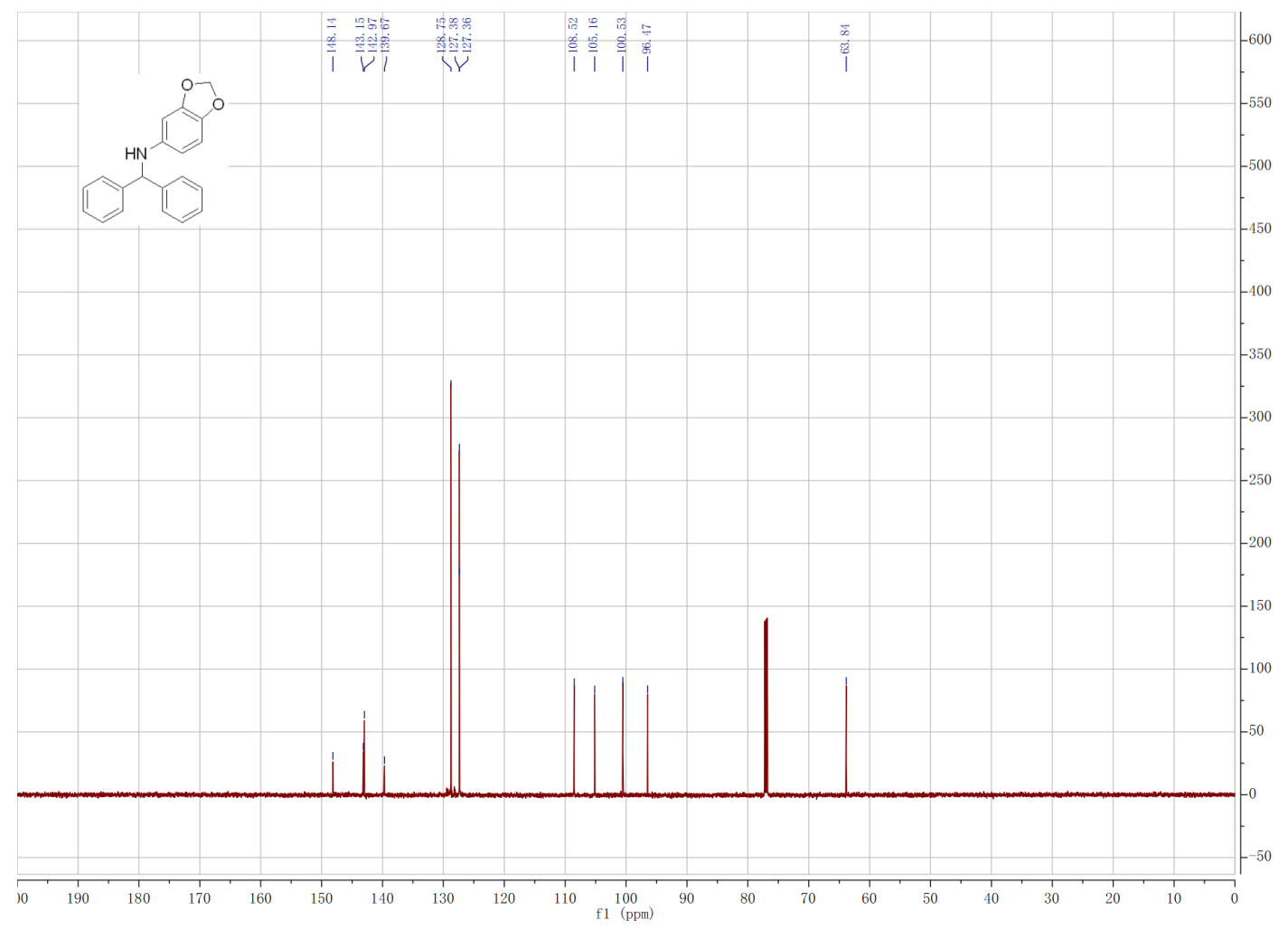


$\mathrm{N}$-(Bis(4-methoxyphenyl)methyl)aniline (10r)

${ }^{1} \mathrm{H}$ NMR (600 MHz, Chloroform-d)

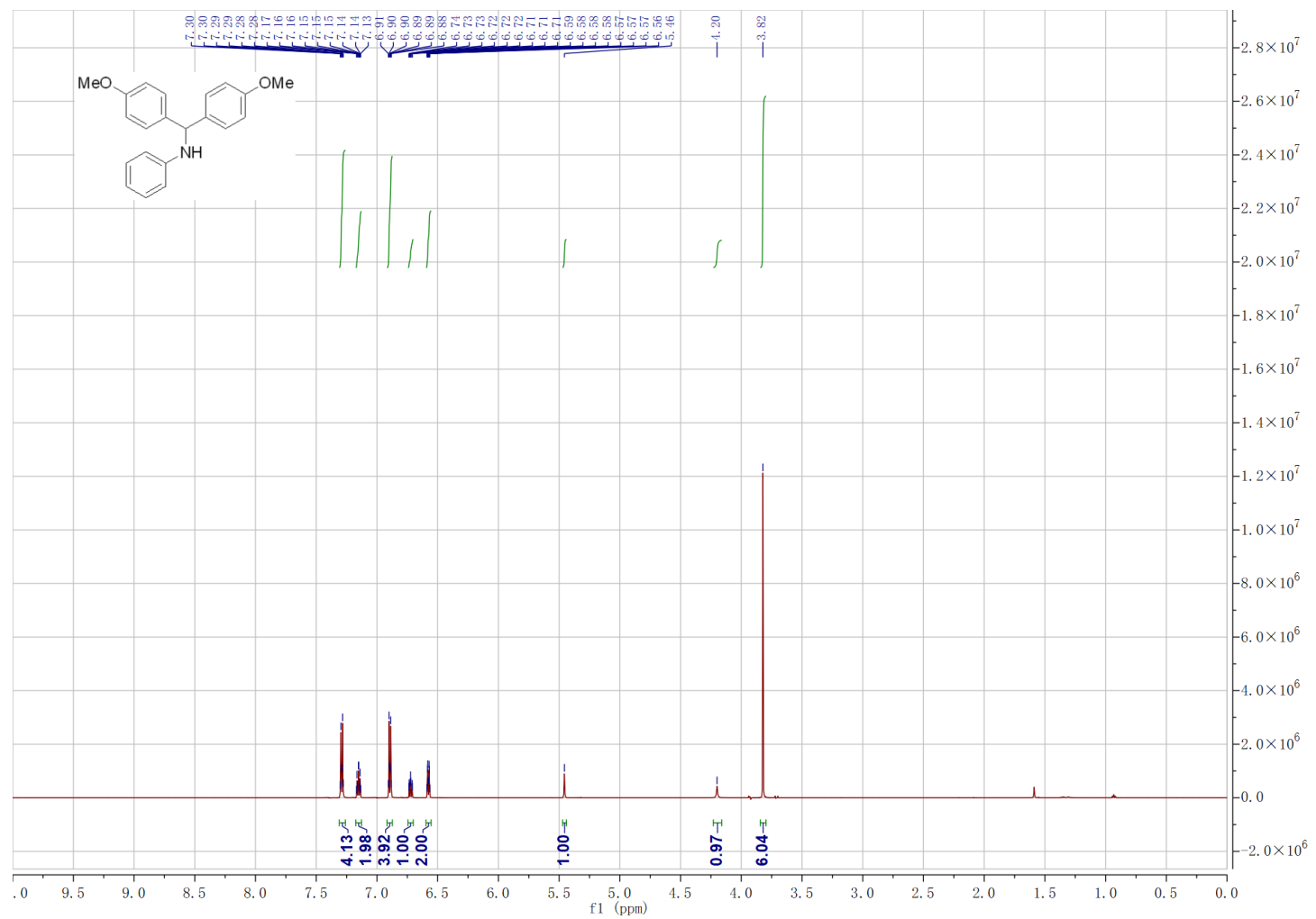

${ }^{13} \mathrm{C}$ NMR (151 MHz, Chloroform- $d$ )

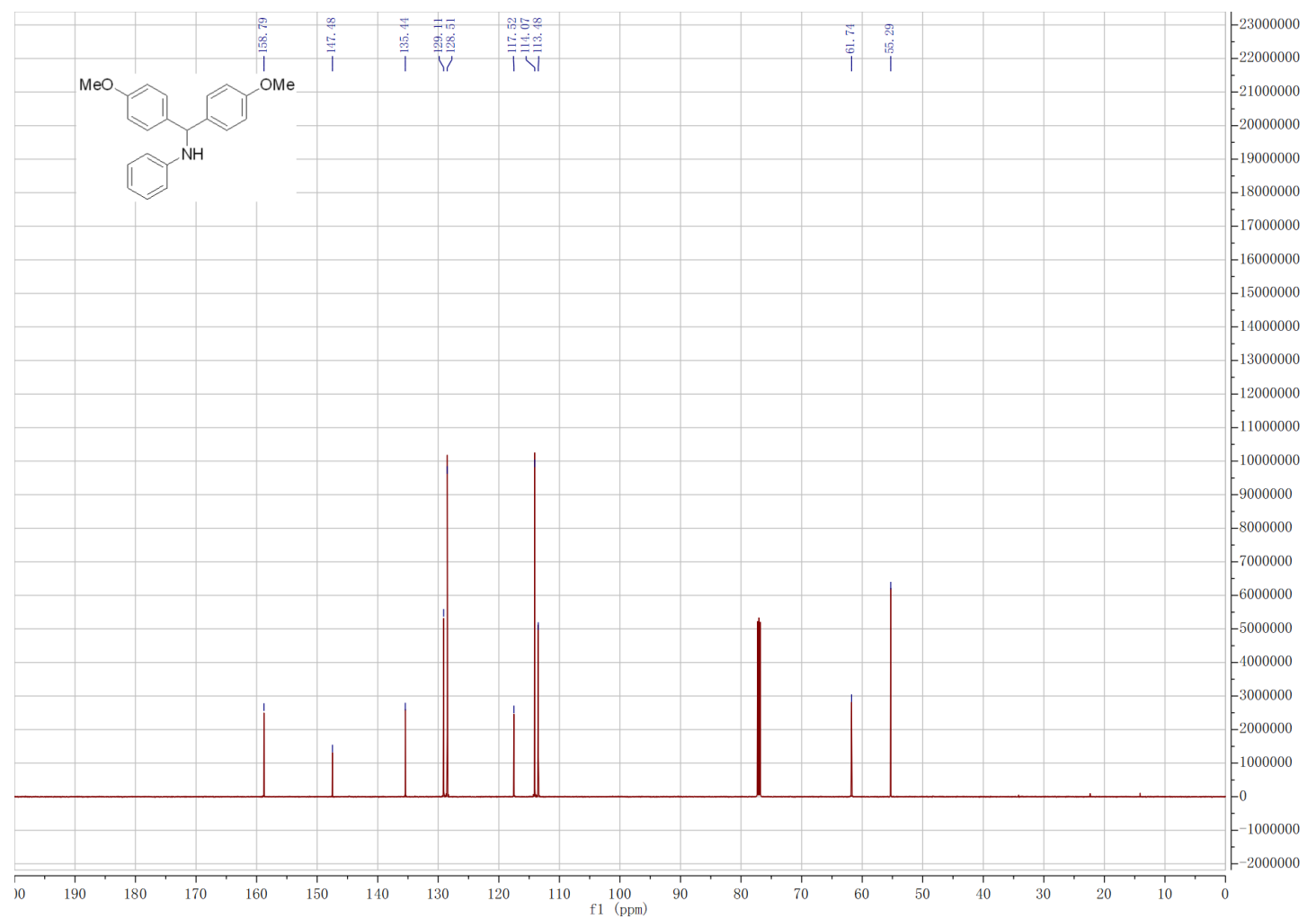


$\mathrm{N}$-((4-Chlorophenyl)(4-methoxyphenyl)methyl)aniline (10s)

${ }^{1} \mathrm{H}$ NMR (600 MHz, Chloroform- $d$ )

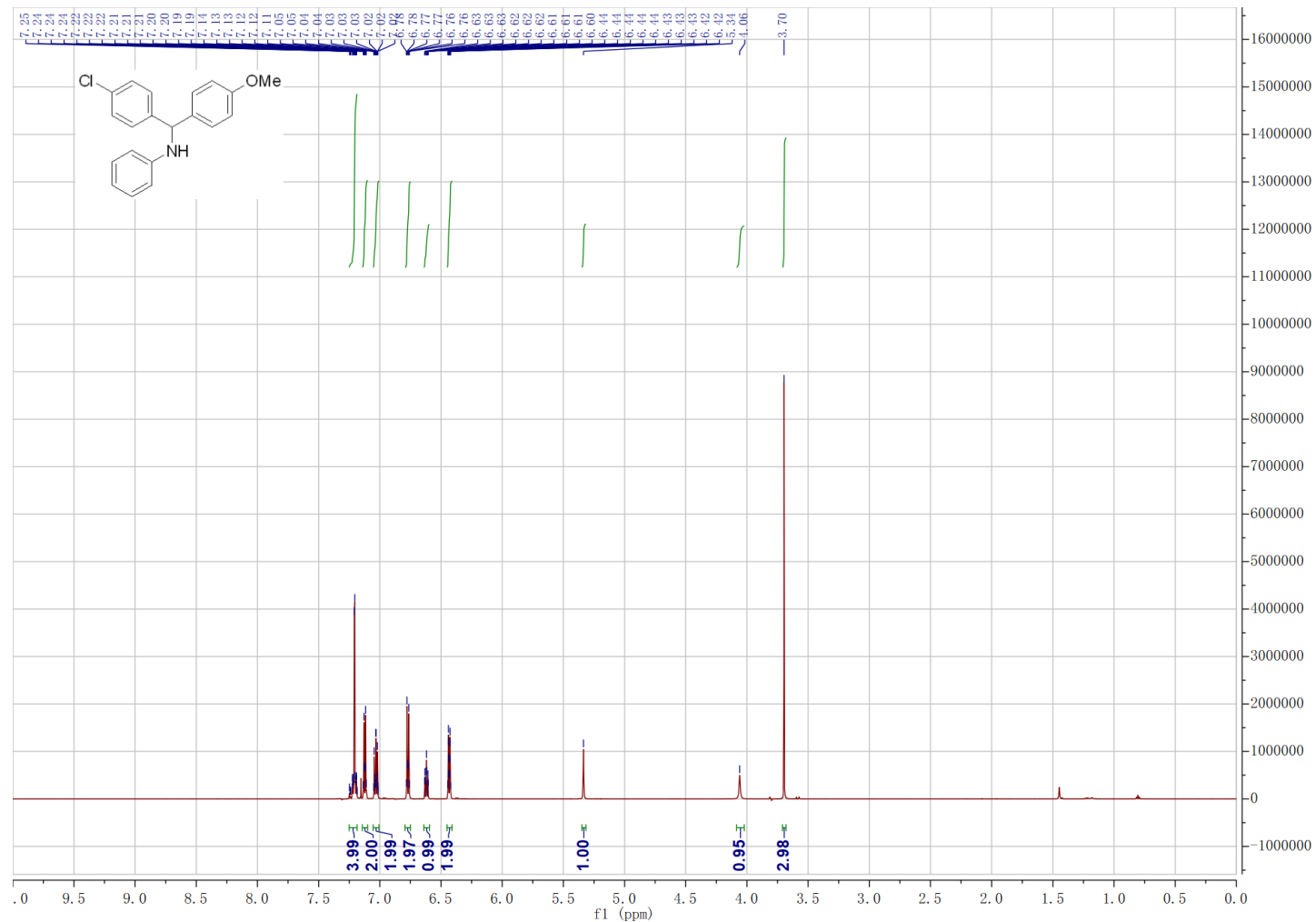

${ }^{13} \mathrm{C}$ NMR (151 MHz, Chloroform- $d$ )

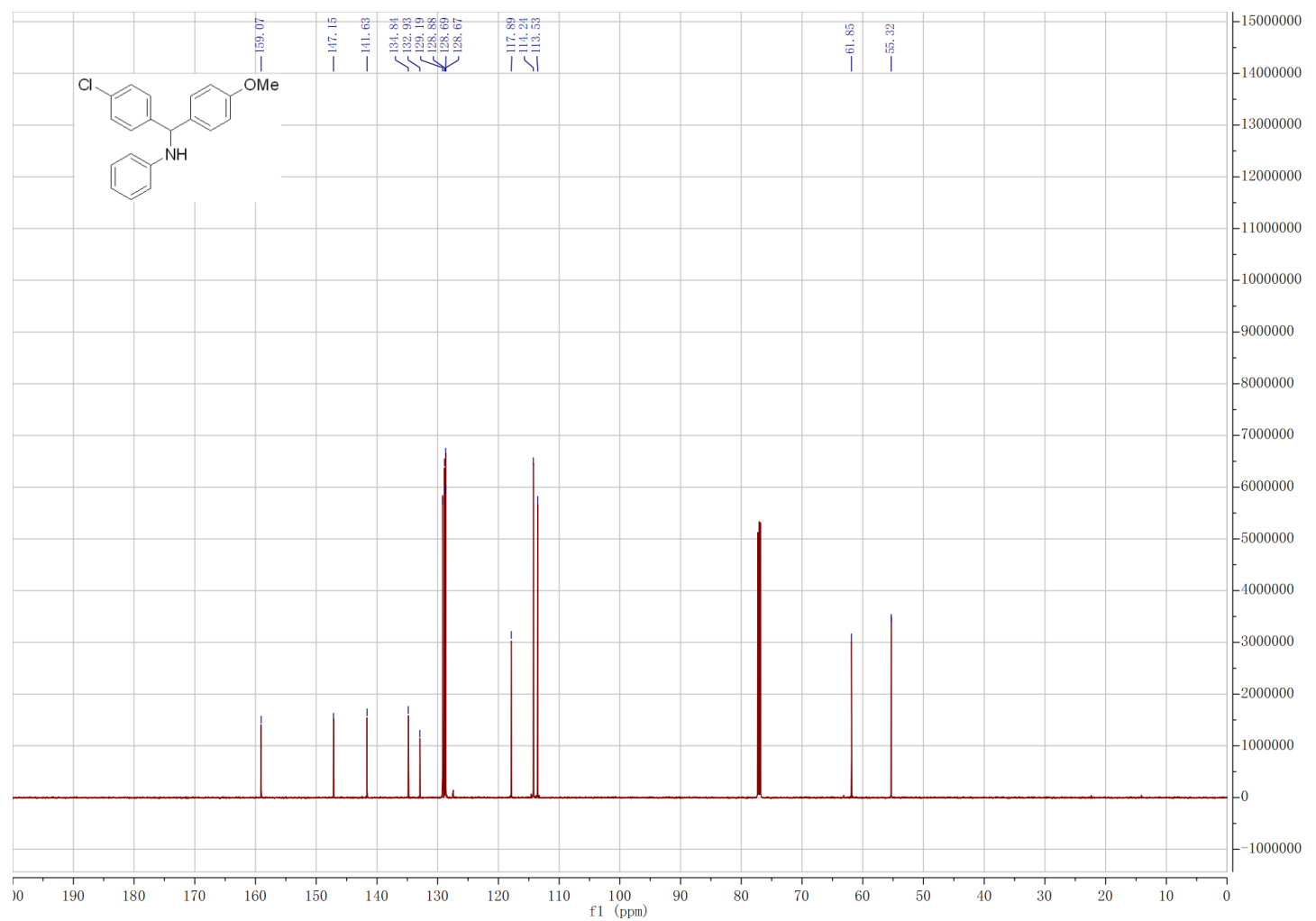


$N$-([1,1'-Biphenyl]-4-yl(phenyl)methyl)aniline (10t)

${ }^{1} \mathrm{H}$ NMR (600 MHz, Chloroform- $d$ )

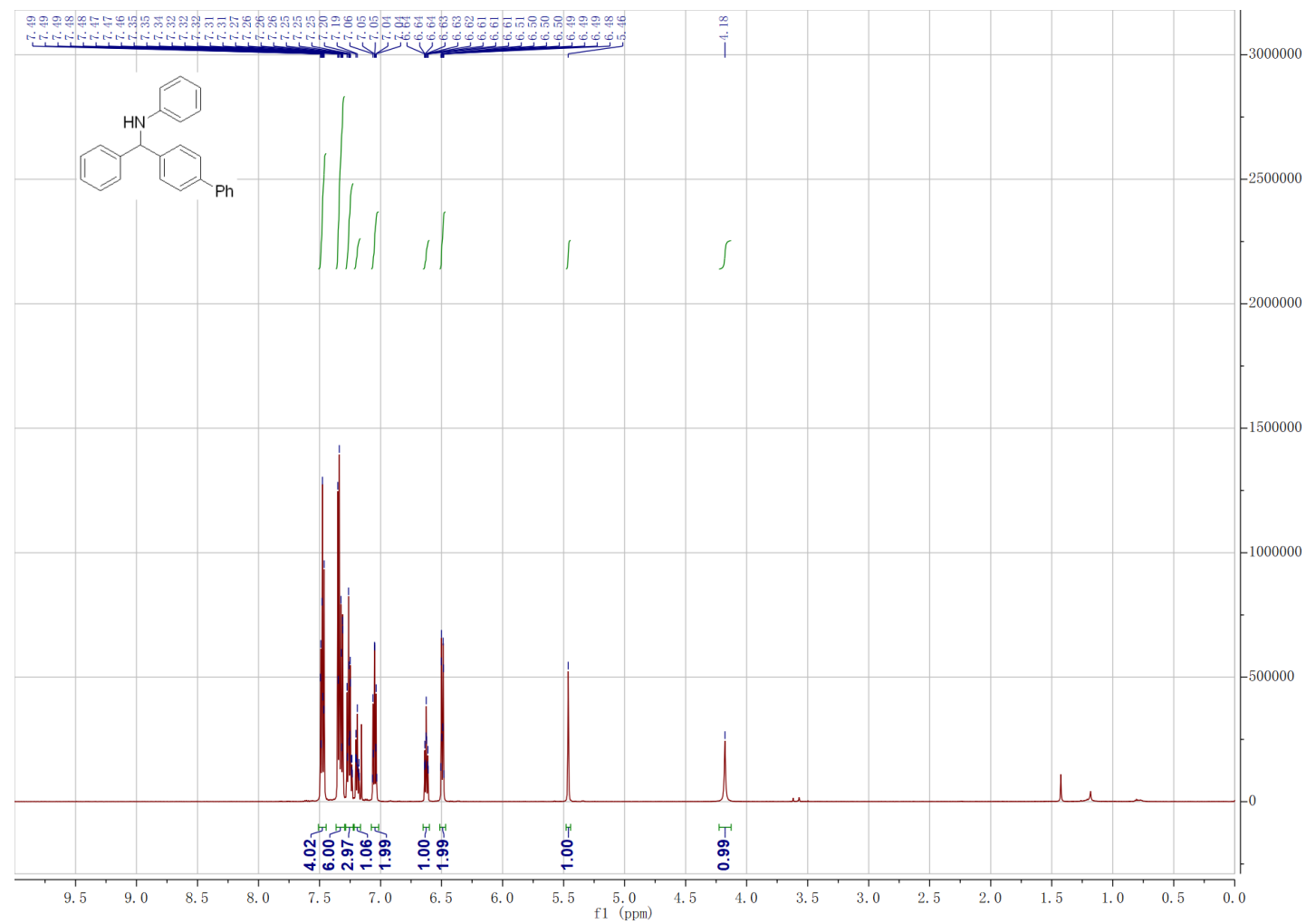

${ }^{13} \mathrm{C}$ NMR (151 MHz, Chloroform- $d$ )

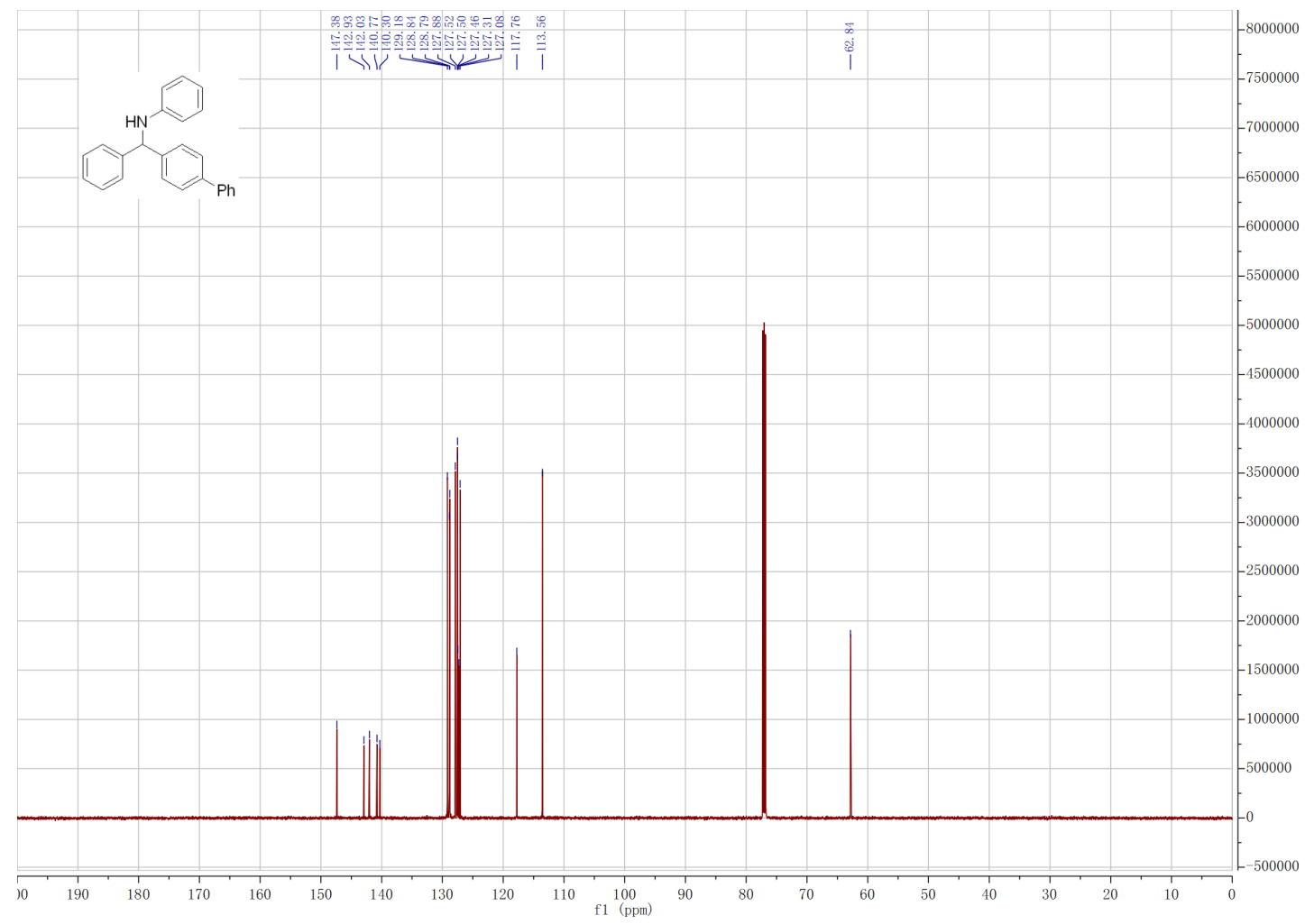


$N$-(Phenyl(m-tolyl)methyl)aniline (10u)

${ }^{1} \mathrm{H}$ NMR (600 MHz, Chloroform- $d$ )

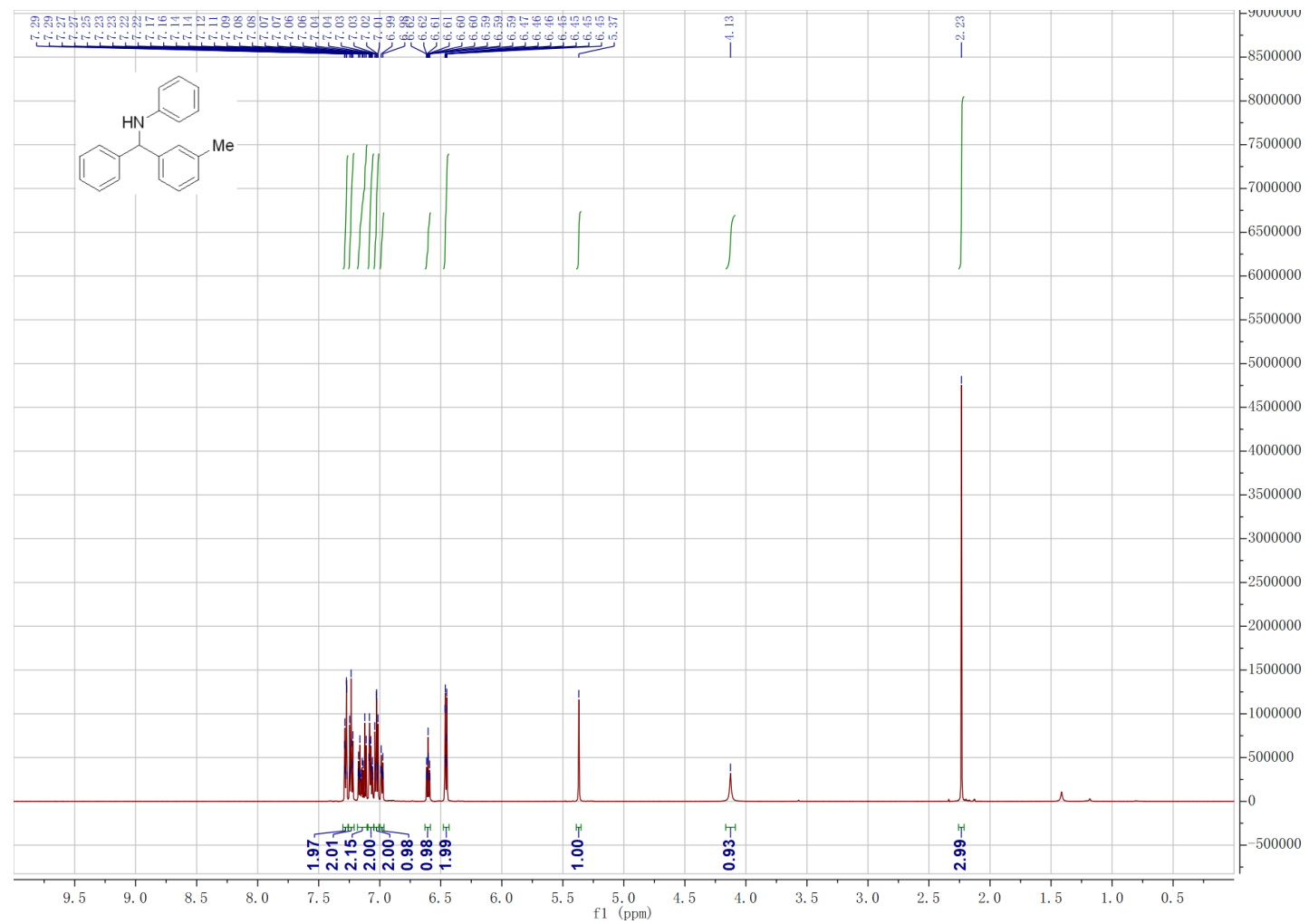

${ }^{13} \mathrm{C}$ NMR (151 MHz, Chloroform- $d$ )

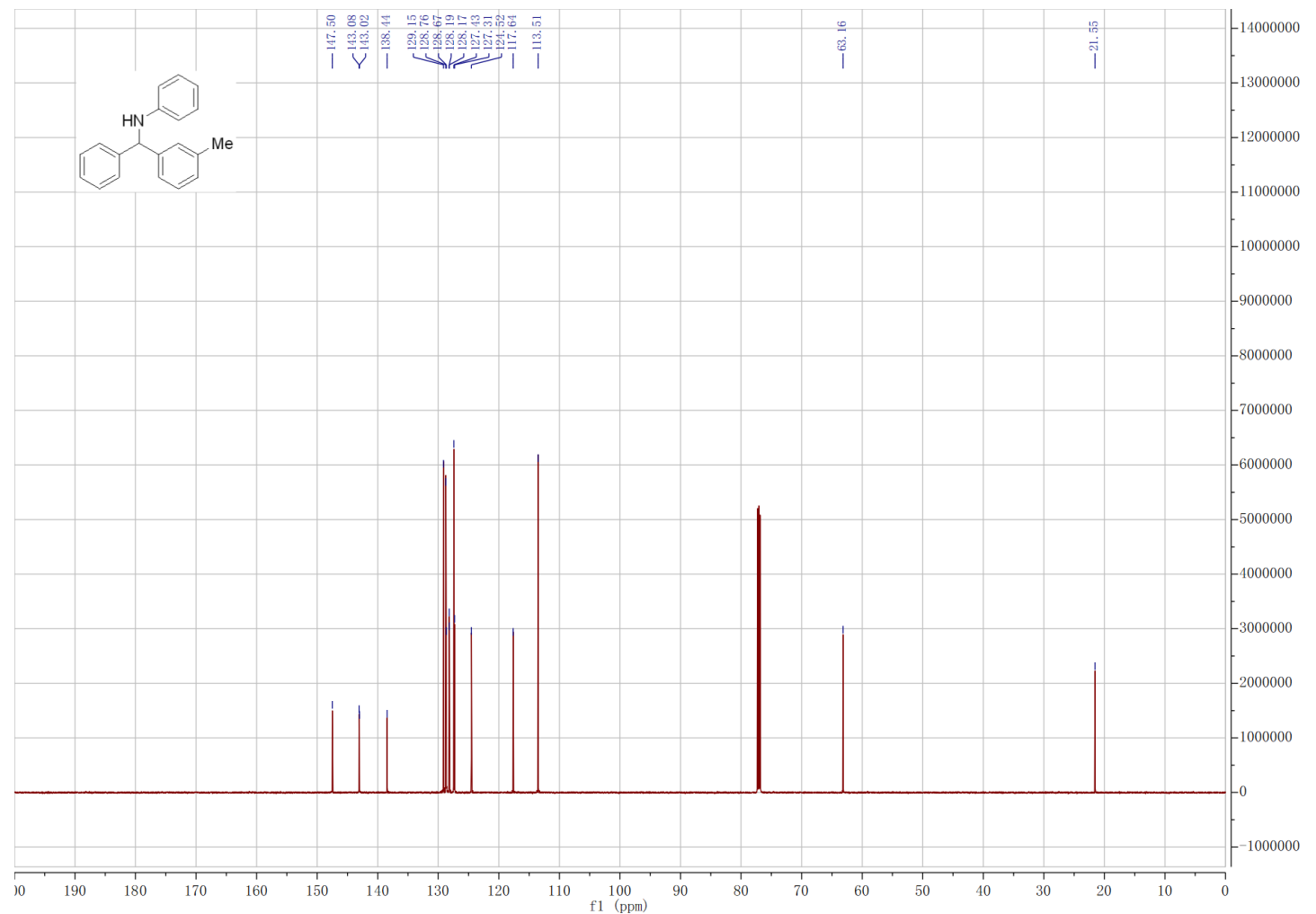


$N$-(Phenyl(o-tolyl)methyl)aniline (10v)

${ }^{1} \mathrm{H}$ NMR (600 MHz, Chloroform- $d$ )

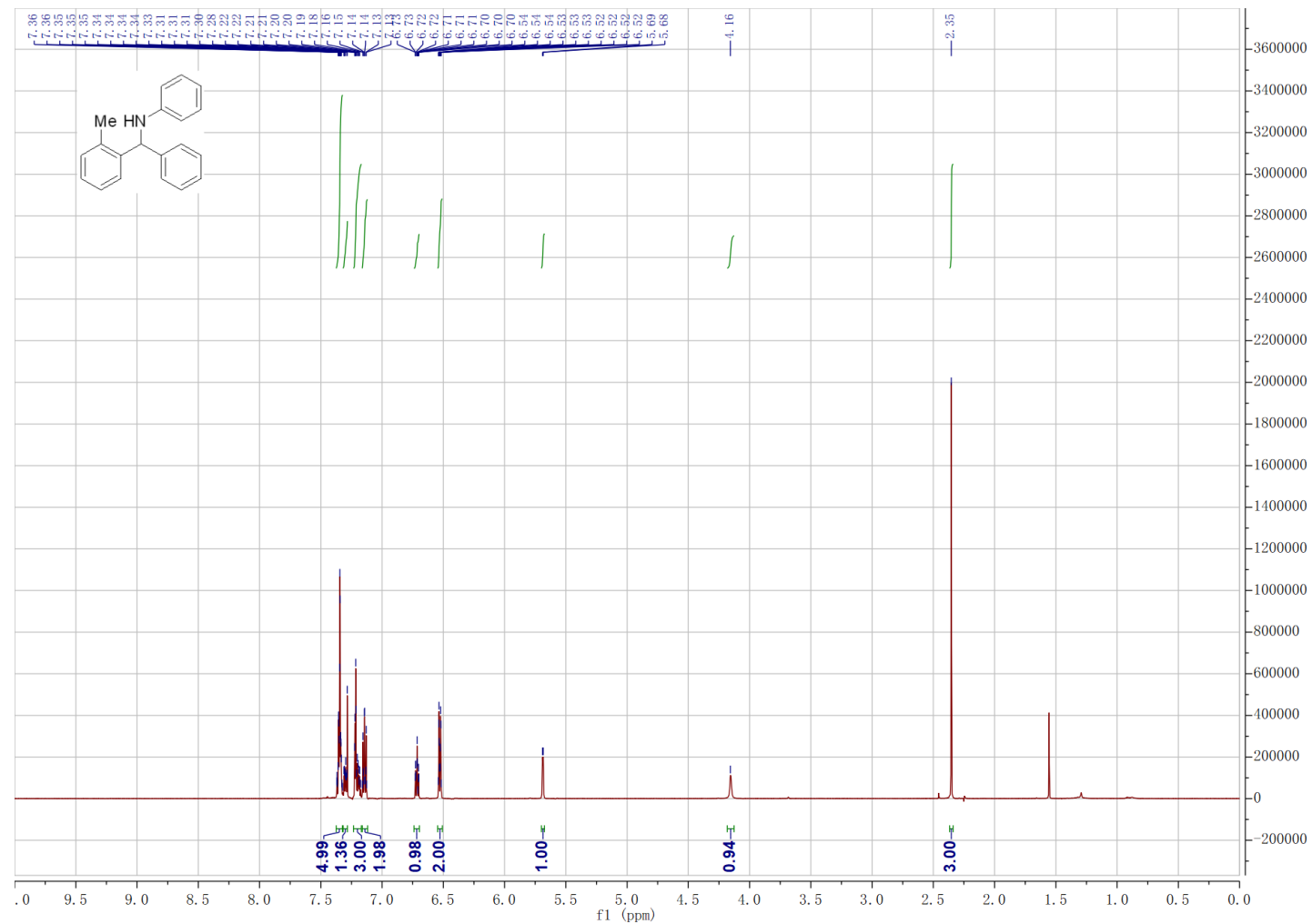

${ }^{13} \mathrm{C}$ NMR (151 MHz, Chloroform- $d$ )

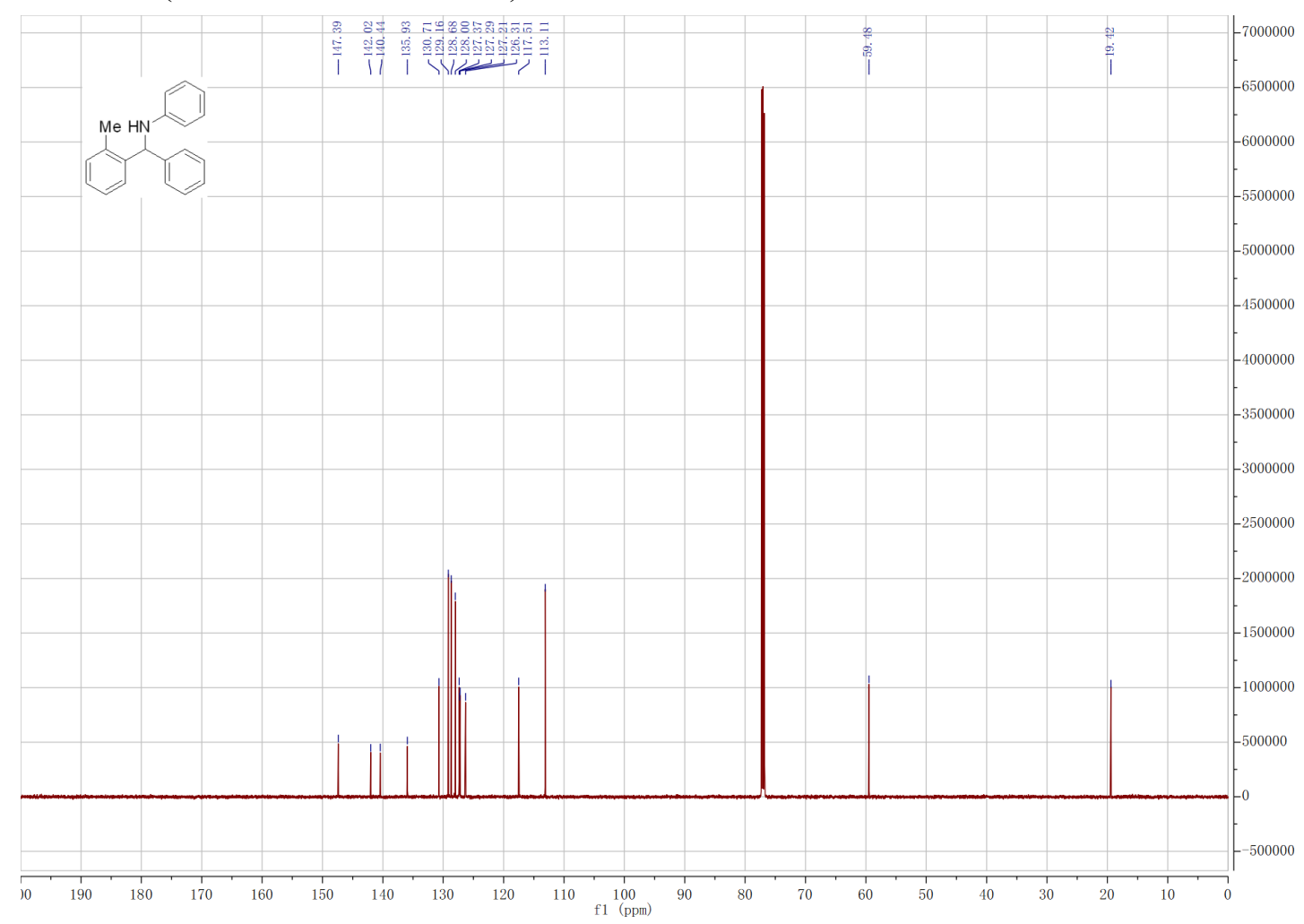


N-((2-Chlorophenyl)(phenyl)methyl)aniline (10w)

${ }^{1} \mathrm{H}$ NMR (600 MHz, Chloroform- $d$ )

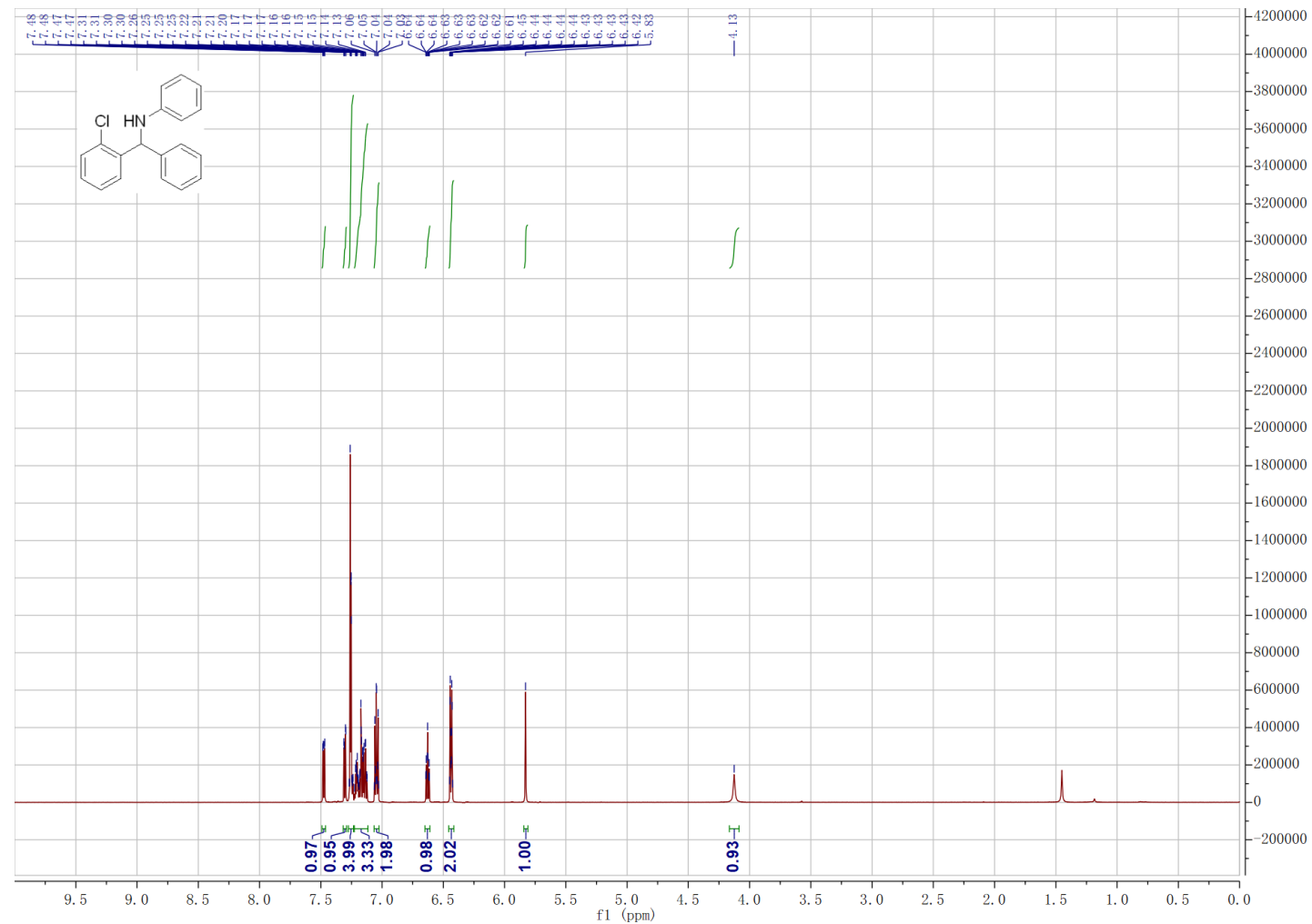

${ }^{13} \mathrm{C}$ NMR (151 MHz, Chloroform- $d$ )

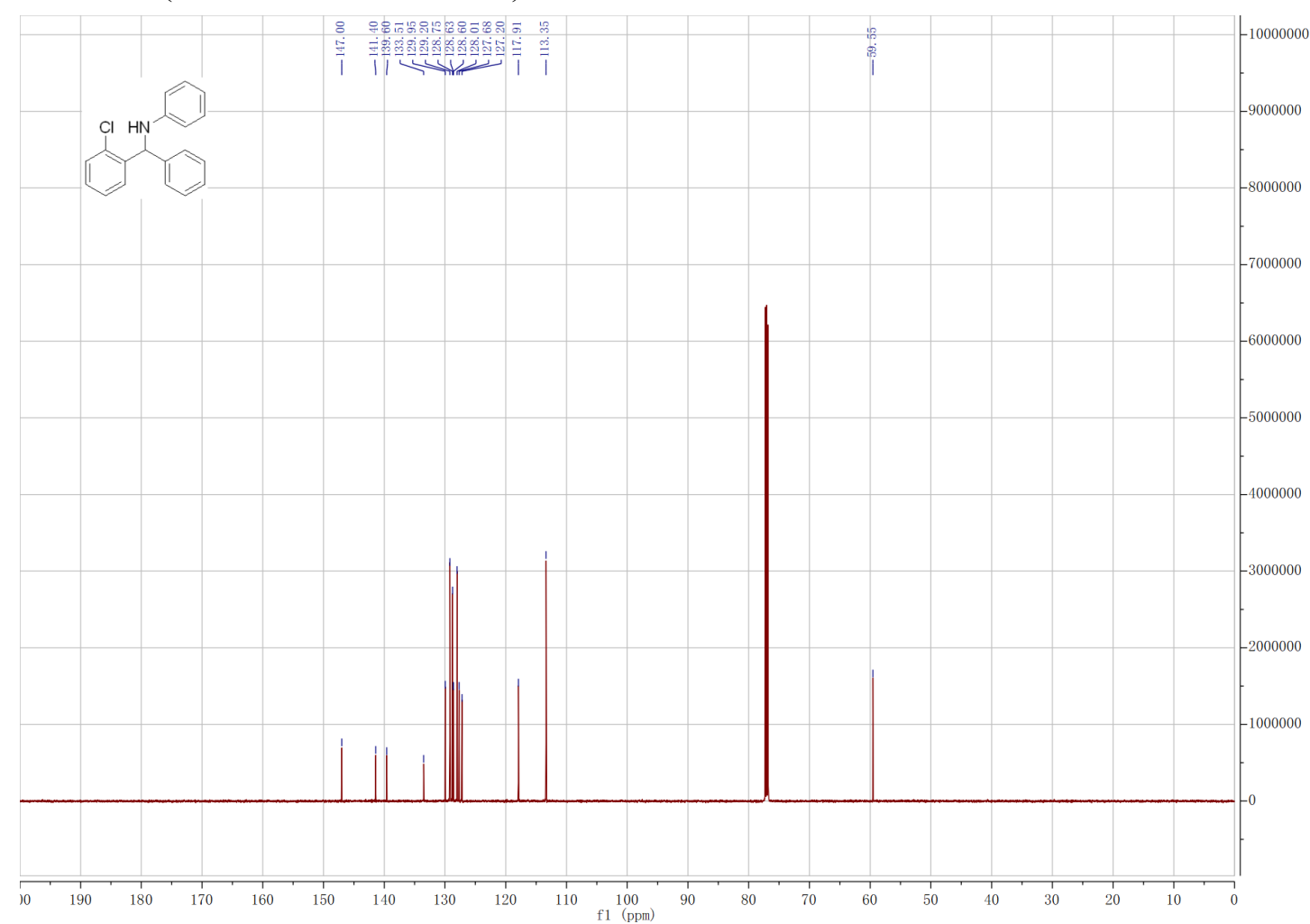


$N$-((4-methoxyphenyl)(3-nitrophenyl)methyl)aniline (10x)

${ }^{1} \mathrm{H}$ NMR (600 MHz, Chloroform- $d$ )

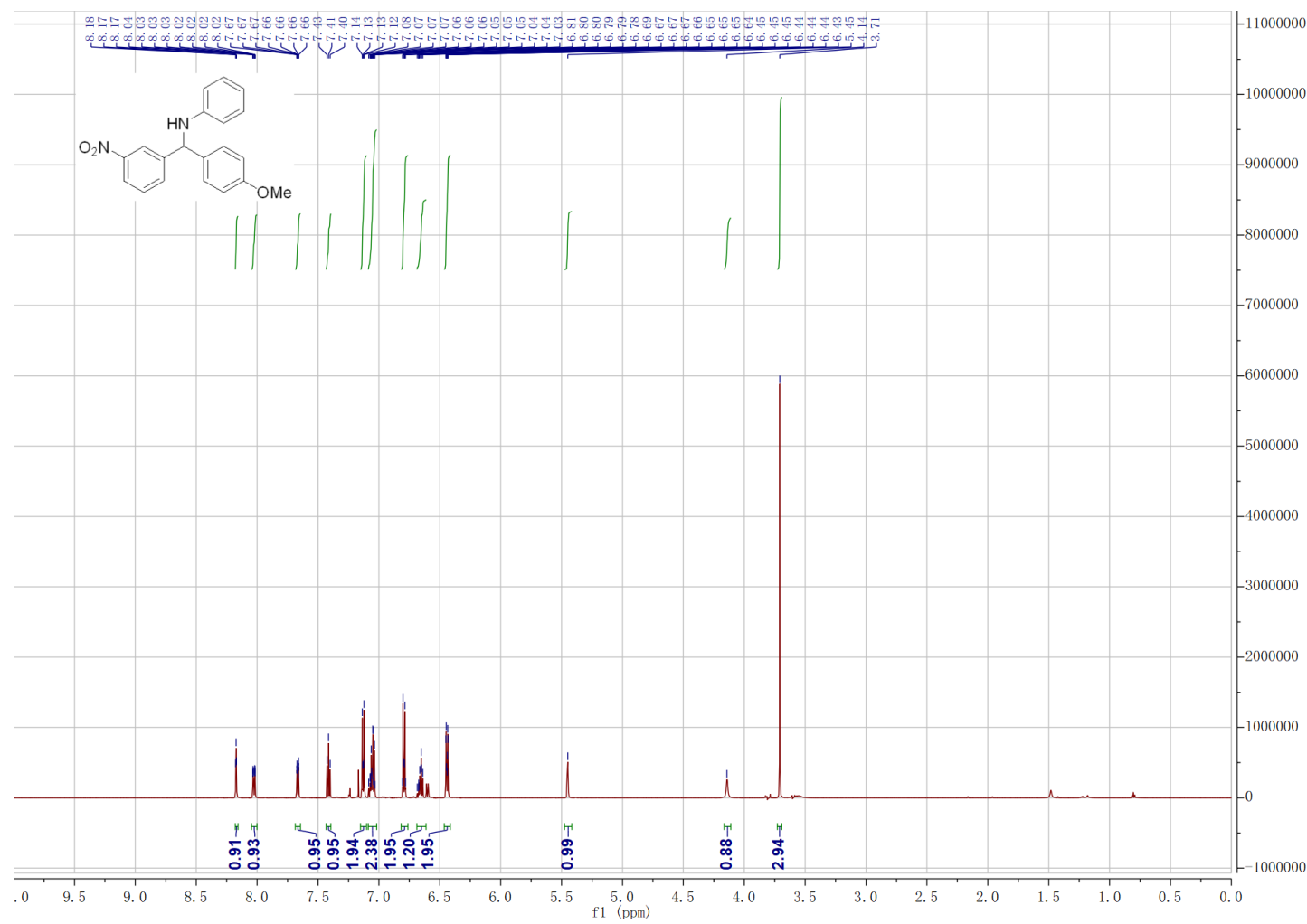

${ }^{13} \mathrm{C}$ NMR (151 MHz, Chloroform- $d$ )

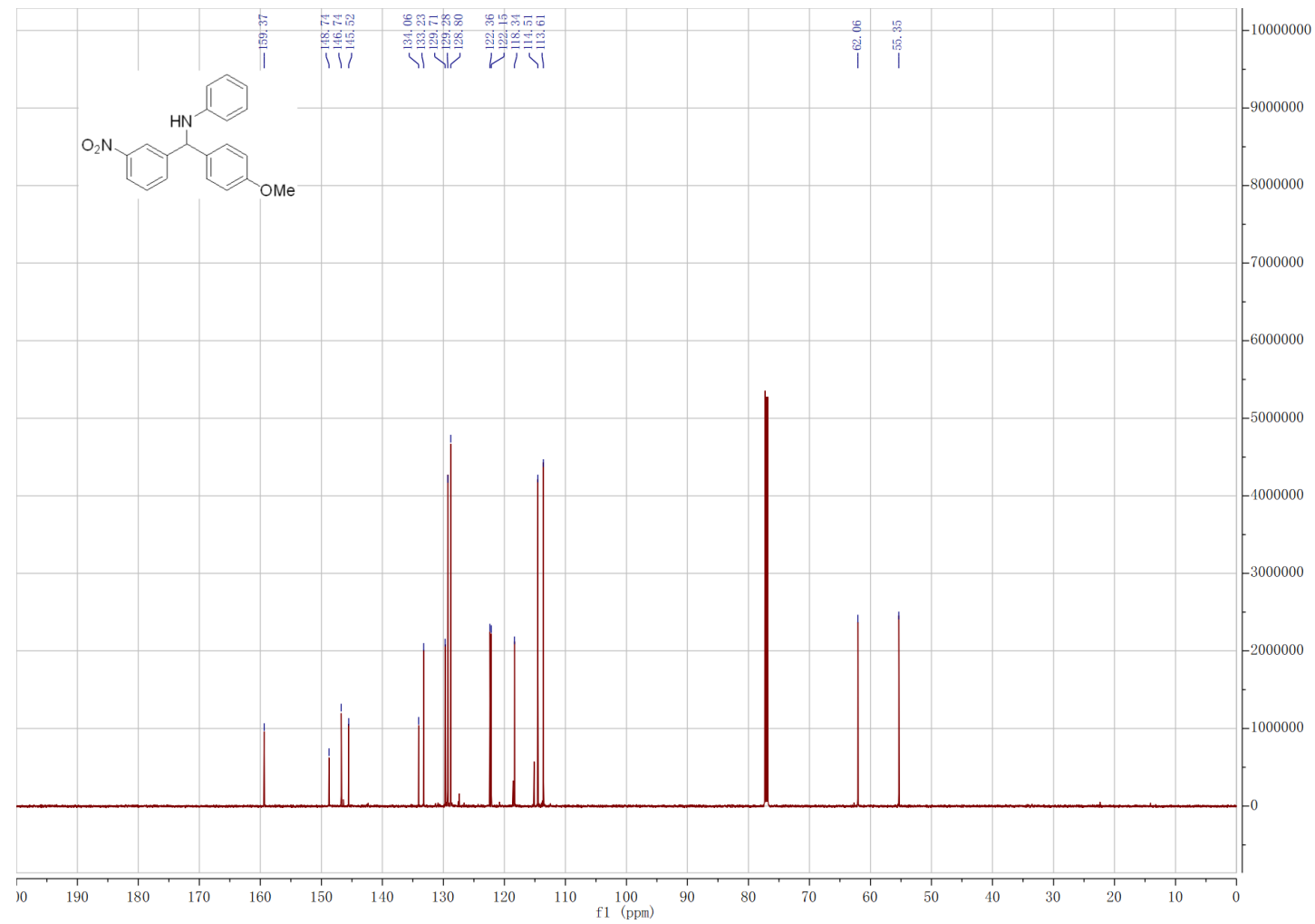


$N$-Benzhydryl- $N$-methylaniline (12a)

${ }^{1} \mathrm{H}$ NMR (600 MHz, Chloroform- $d$ )

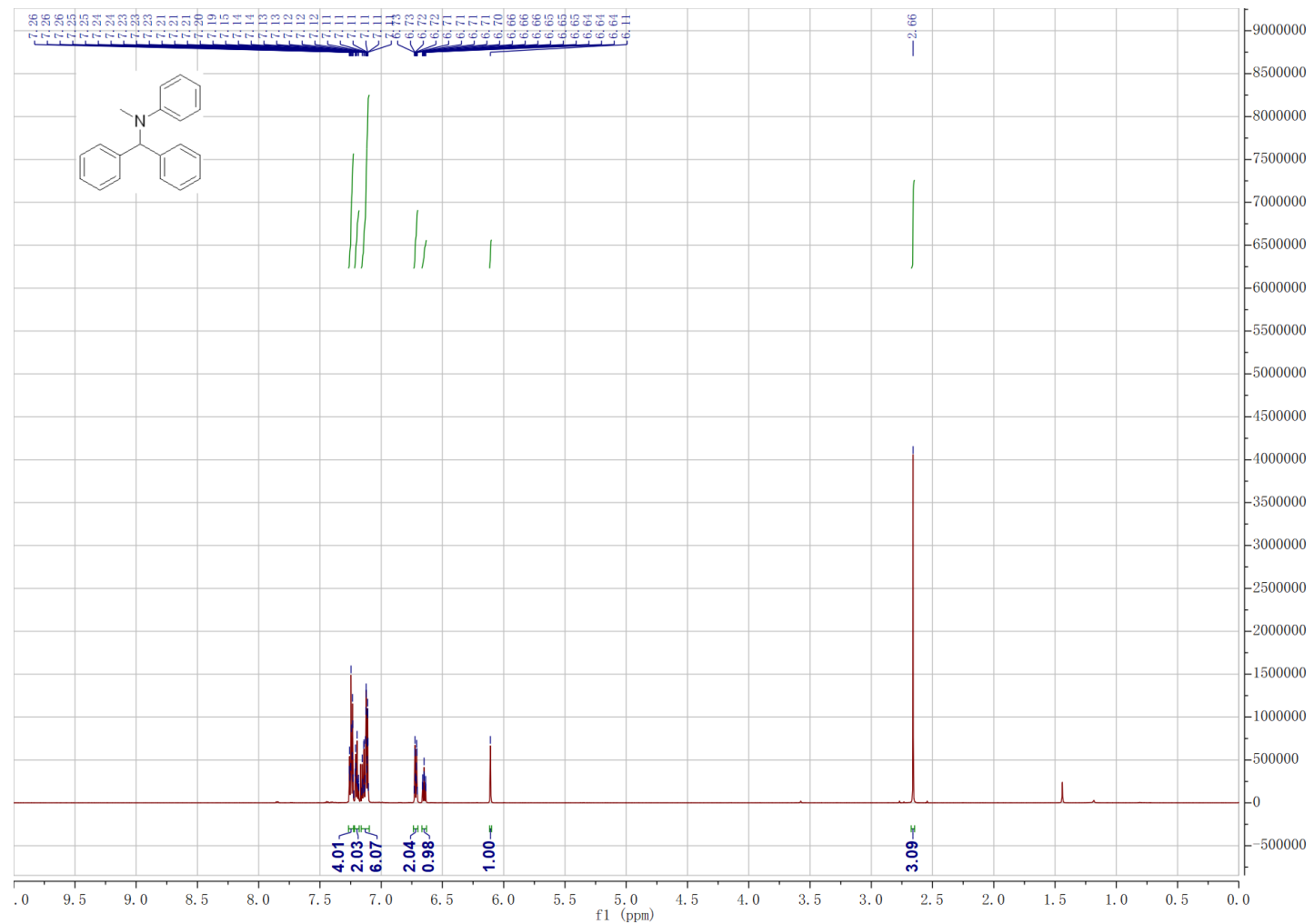

${ }^{13} \mathrm{C}$ NMR (151 MHz, Chloroform- $d$ )

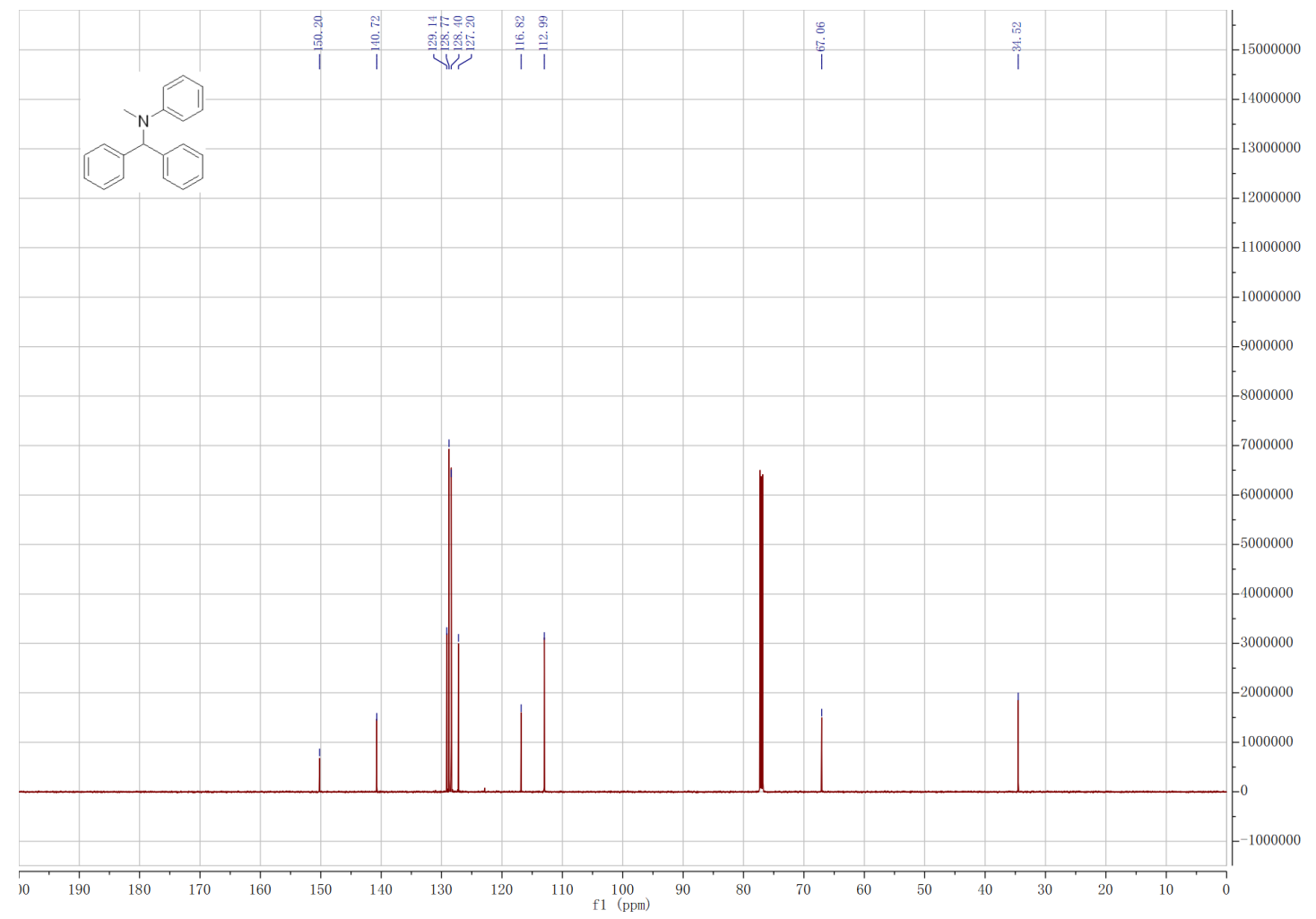


$N$-Benzhydryl- $N$-ethylaniline (12b)

${ }^{1} \mathrm{H}$ NMR (600 MHz, Chloroform- $d$ )

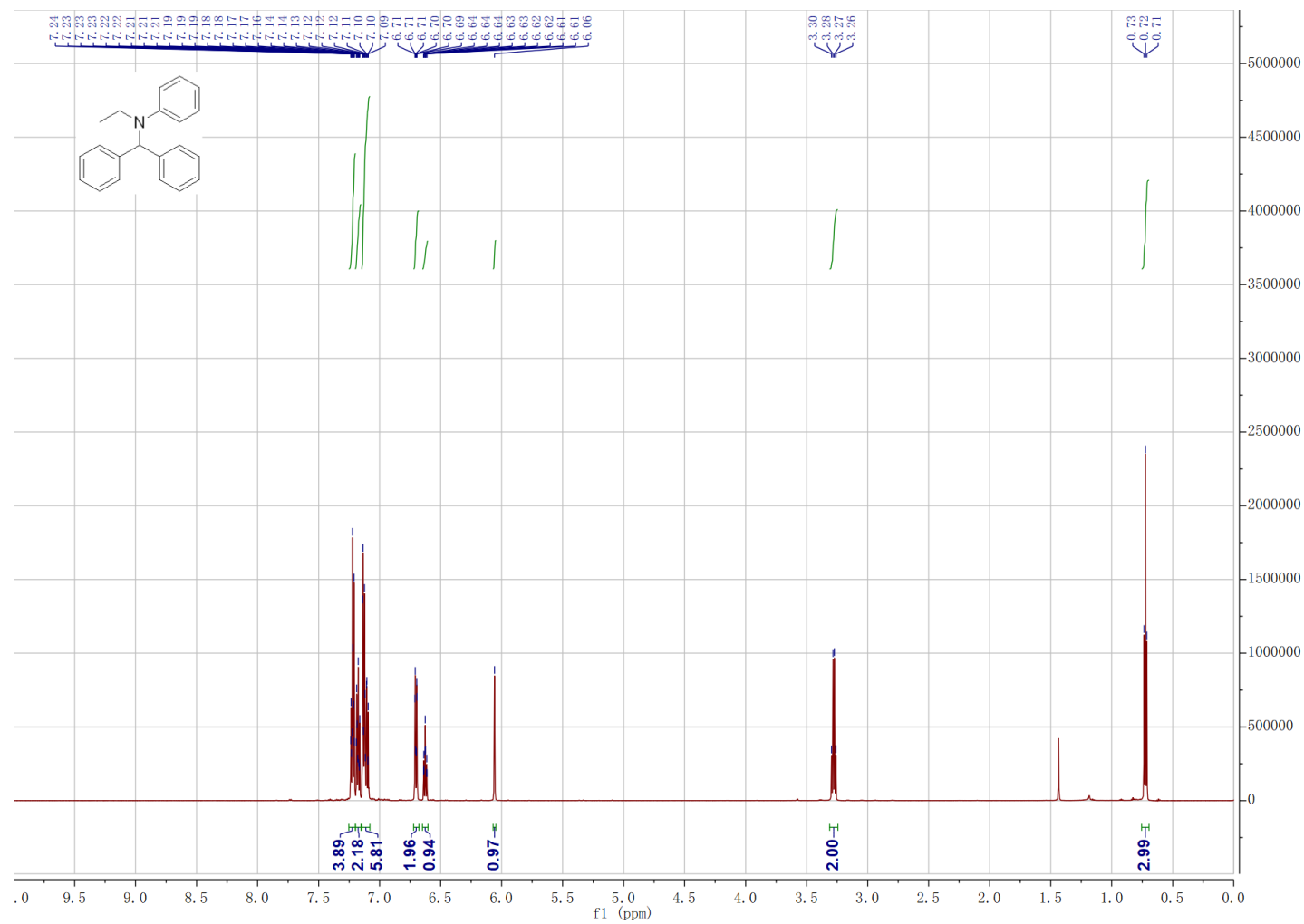

${ }^{13} \mathrm{C}$ NMR (151 MHz, Chloroform- $d$ )

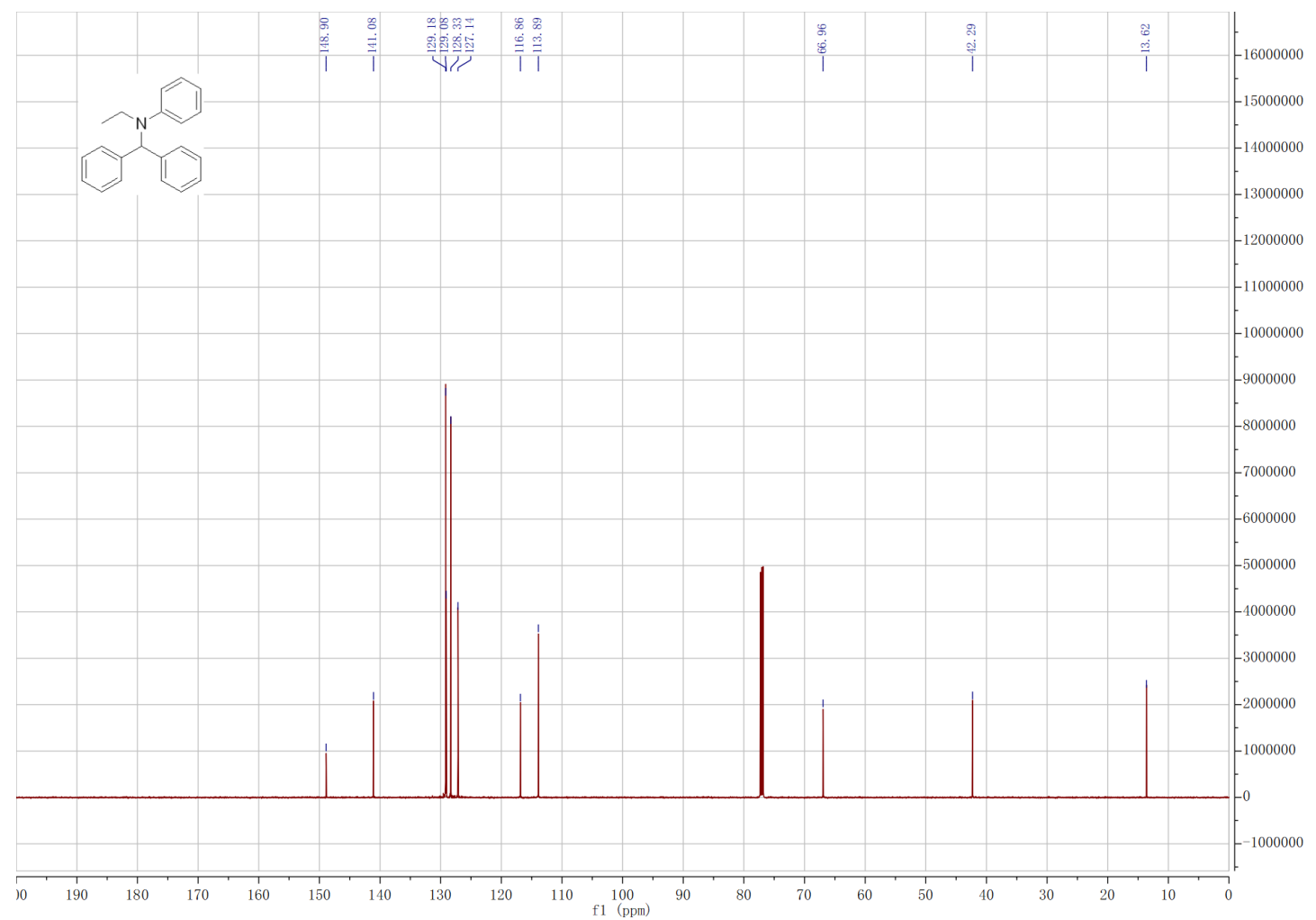


$\boldsymbol{N}$-Benzhydryl-4-methoxy- $\boldsymbol{N}$-methylaniline (12c)

${ }^{1} \mathrm{H}$ NMR (600 MHz, Chloroform-d)

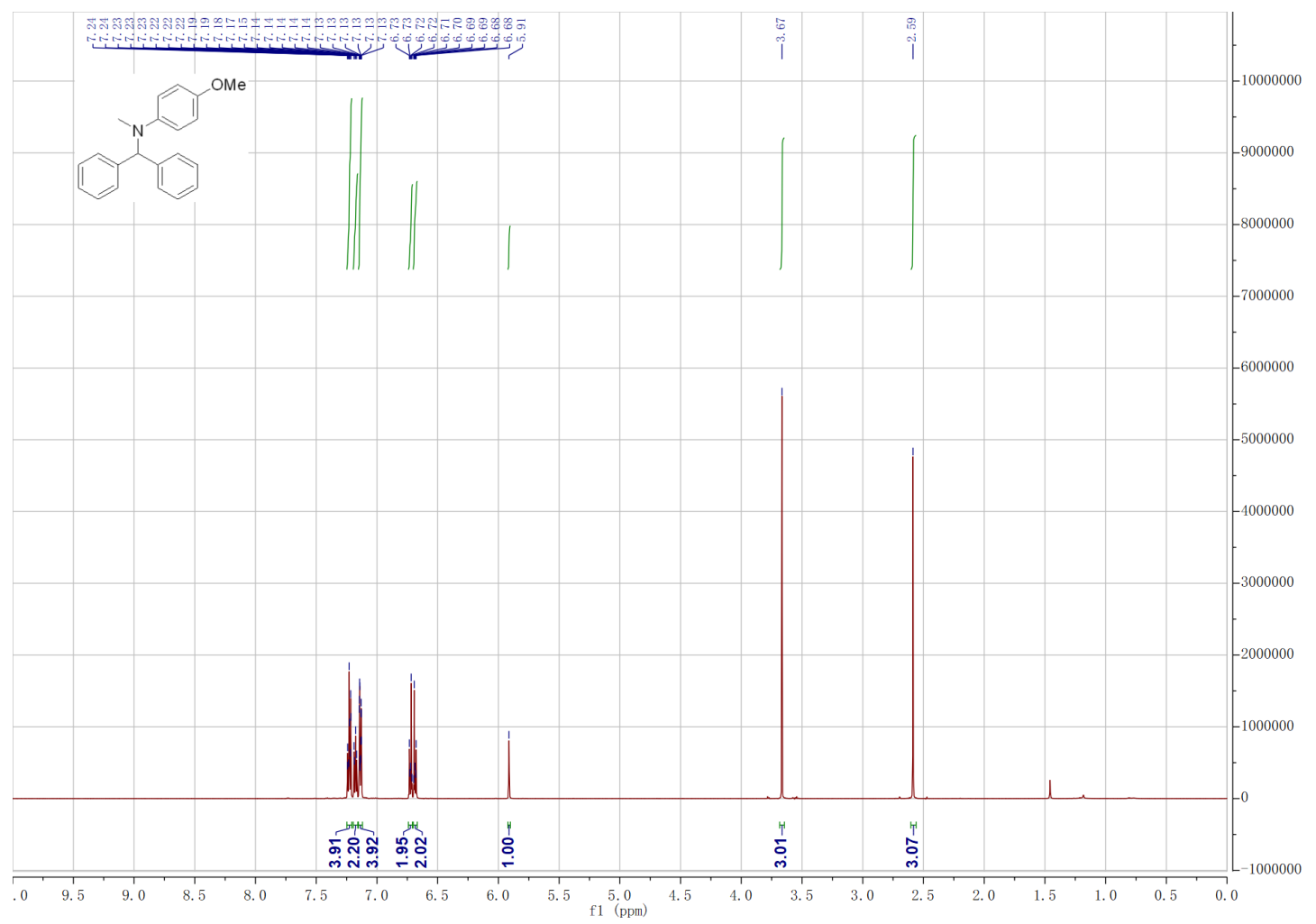

${ }^{13} \mathrm{C}$ NMR (151 MHz, Chloroform- $d$ )

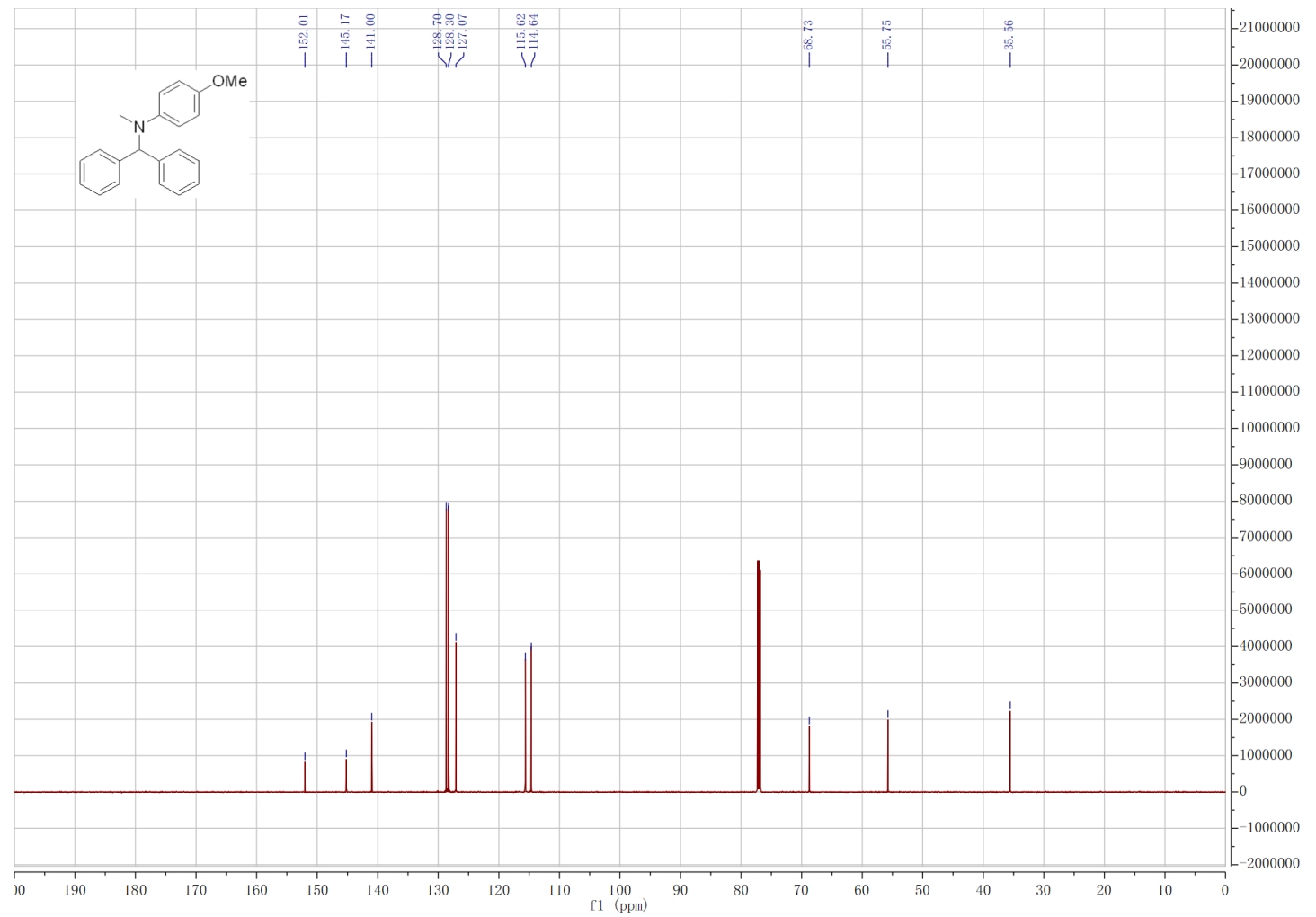


$\boldsymbol{N}$-Benzhydryl-4-chloro- $\boldsymbol{N}$-methylaniline (12d)

${ }^{1}$ H NMR (600 MHz, Chloroform- $d$ )

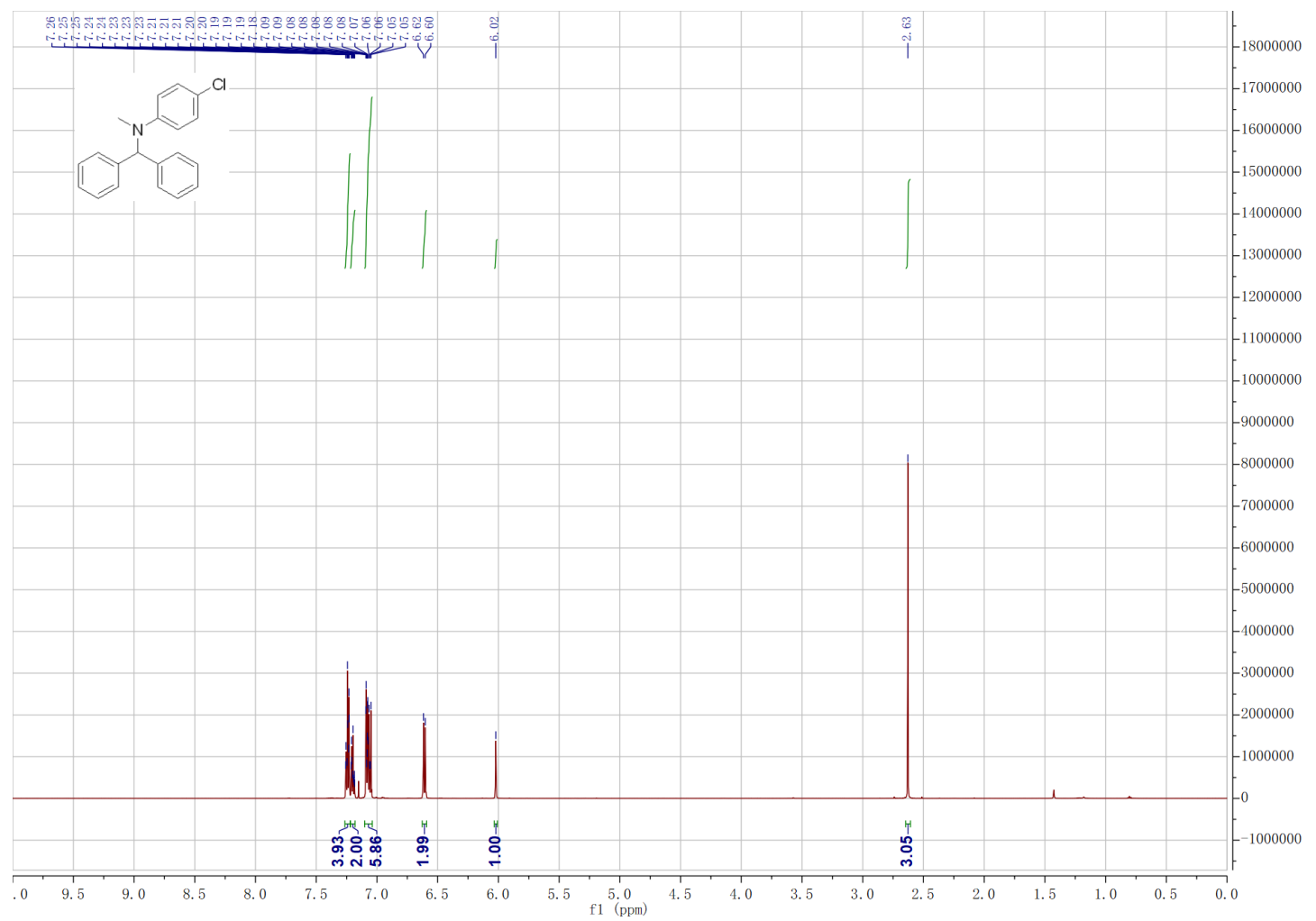

${ }^{13} \mathrm{C}$ NMR (151 MHz, Chloroform- $d$ )

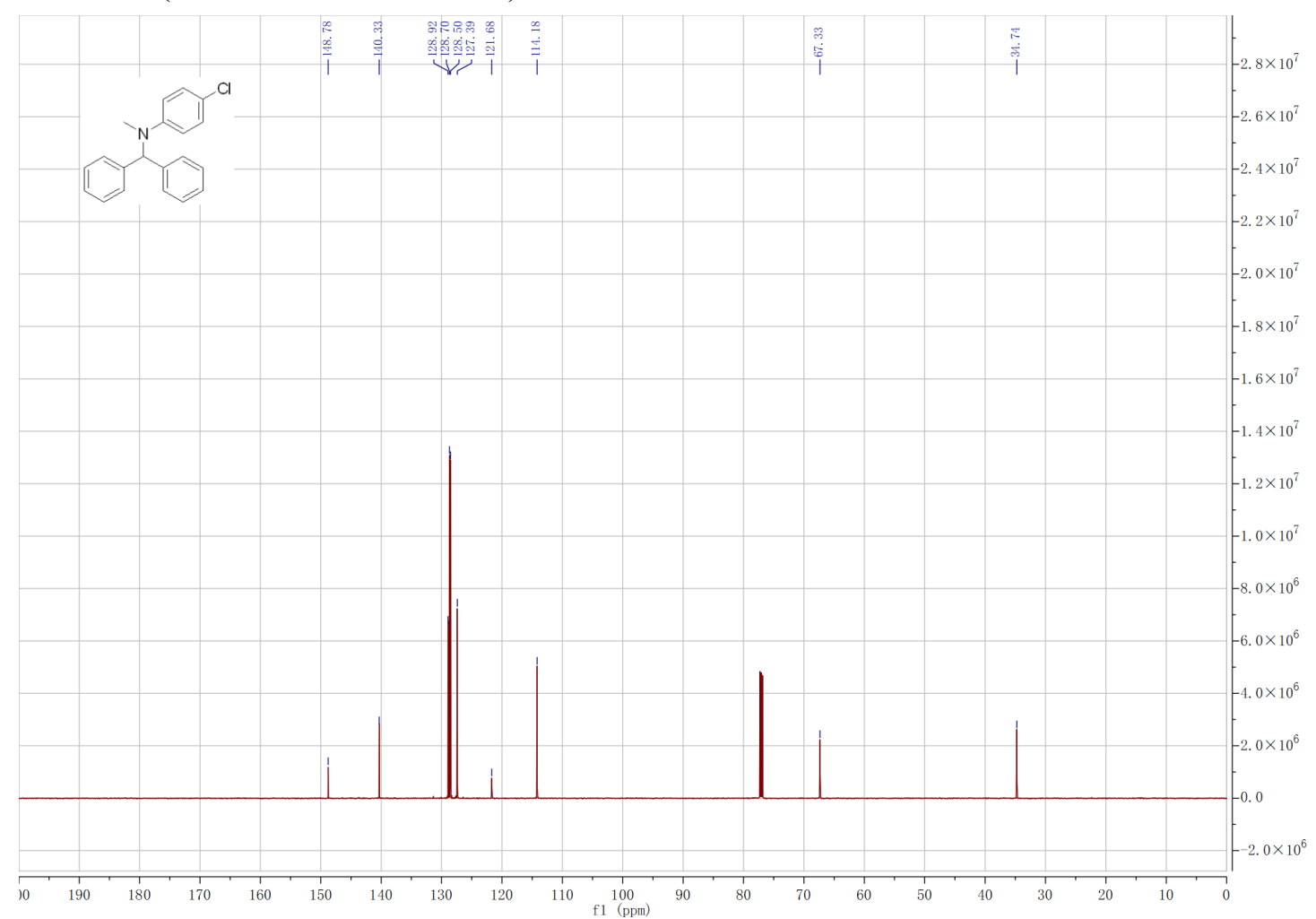


$N$-Benzhydryl- $N, 3$-dimethylaniline (12e)

${ }^{1} \mathrm{H}$ NMR (600 MHz, Chloroform- $d$ )

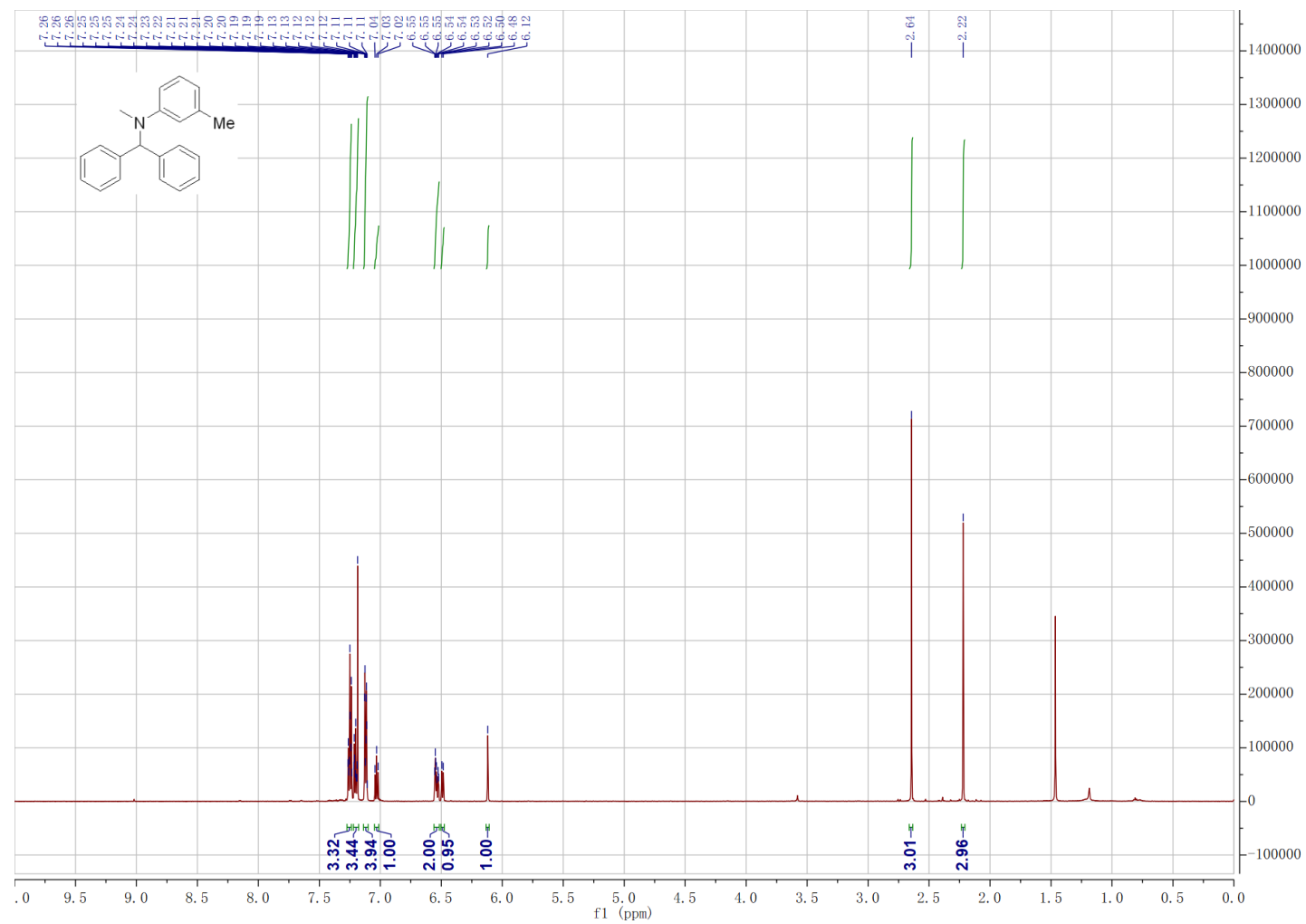

${ }^{13} \mathrm{C}$ NMR (151 MHz, Chloroform- $d$ )

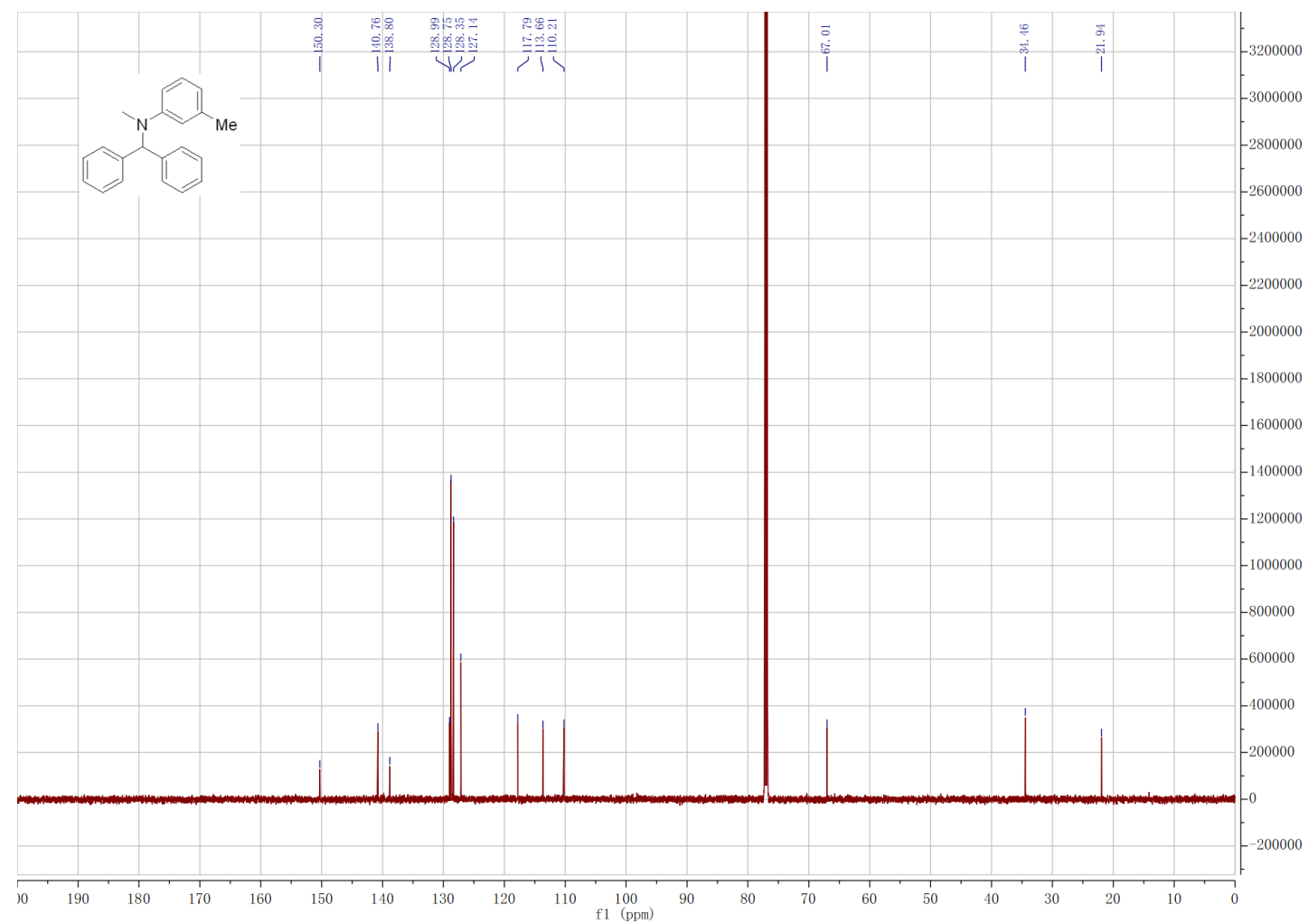


$N$-Benzhydryl- $N$,2-dimethylaniline (12f)

${ }^{1} \mathrm{H}$ NMR (600 MHz, Chloroform- $d$ )

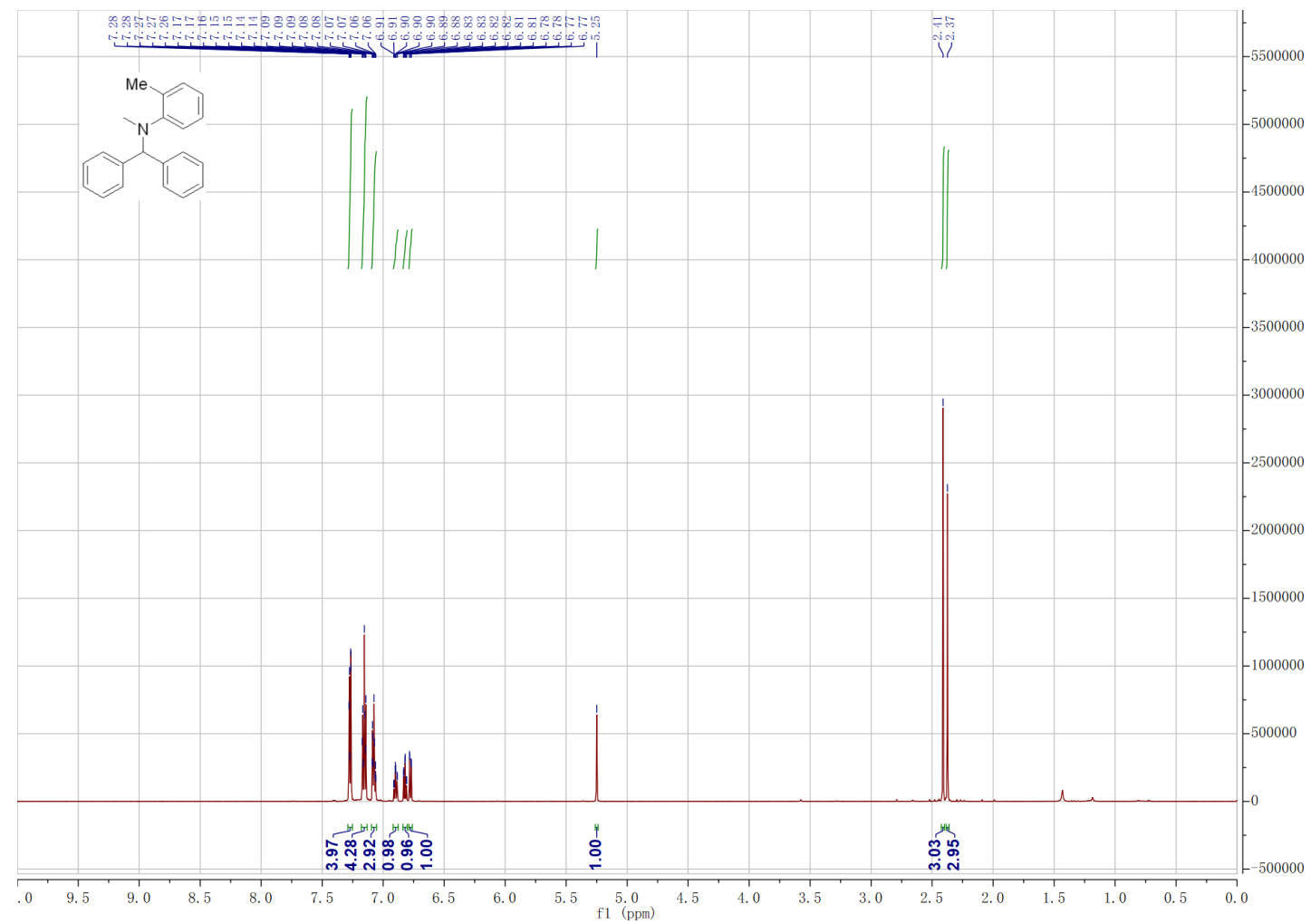

${ }^{13} \mathrm{C}$ NMR (151 MHz, Chloroform- $d$ )

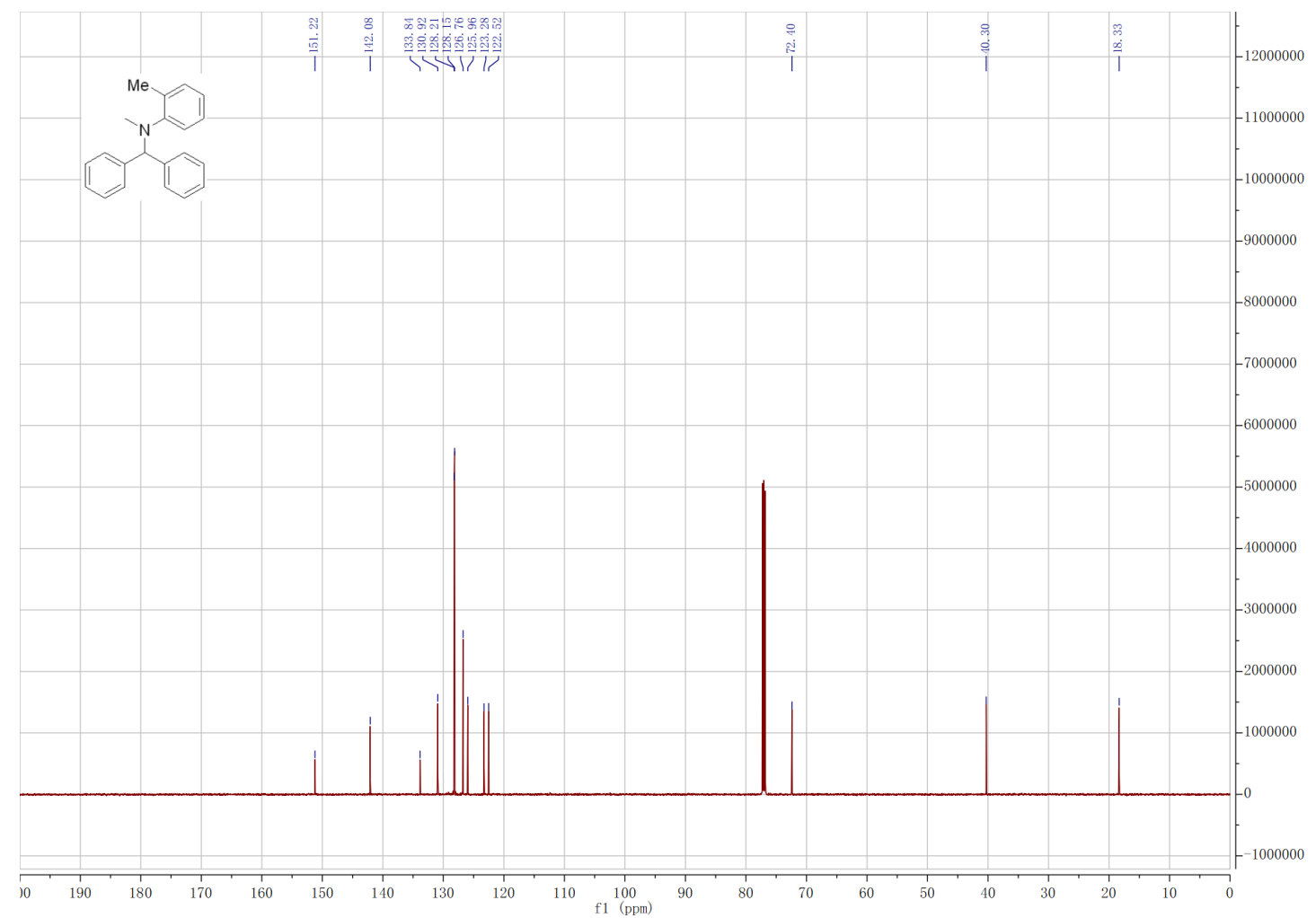


$\boldsymbol{N}$-Benzhydryl- $\boldsymbol{N}$-phenylaniline (12g)

${ }^{1} \mathrm{H}$ NMR (600 MHz, Chloroform- $d$ )

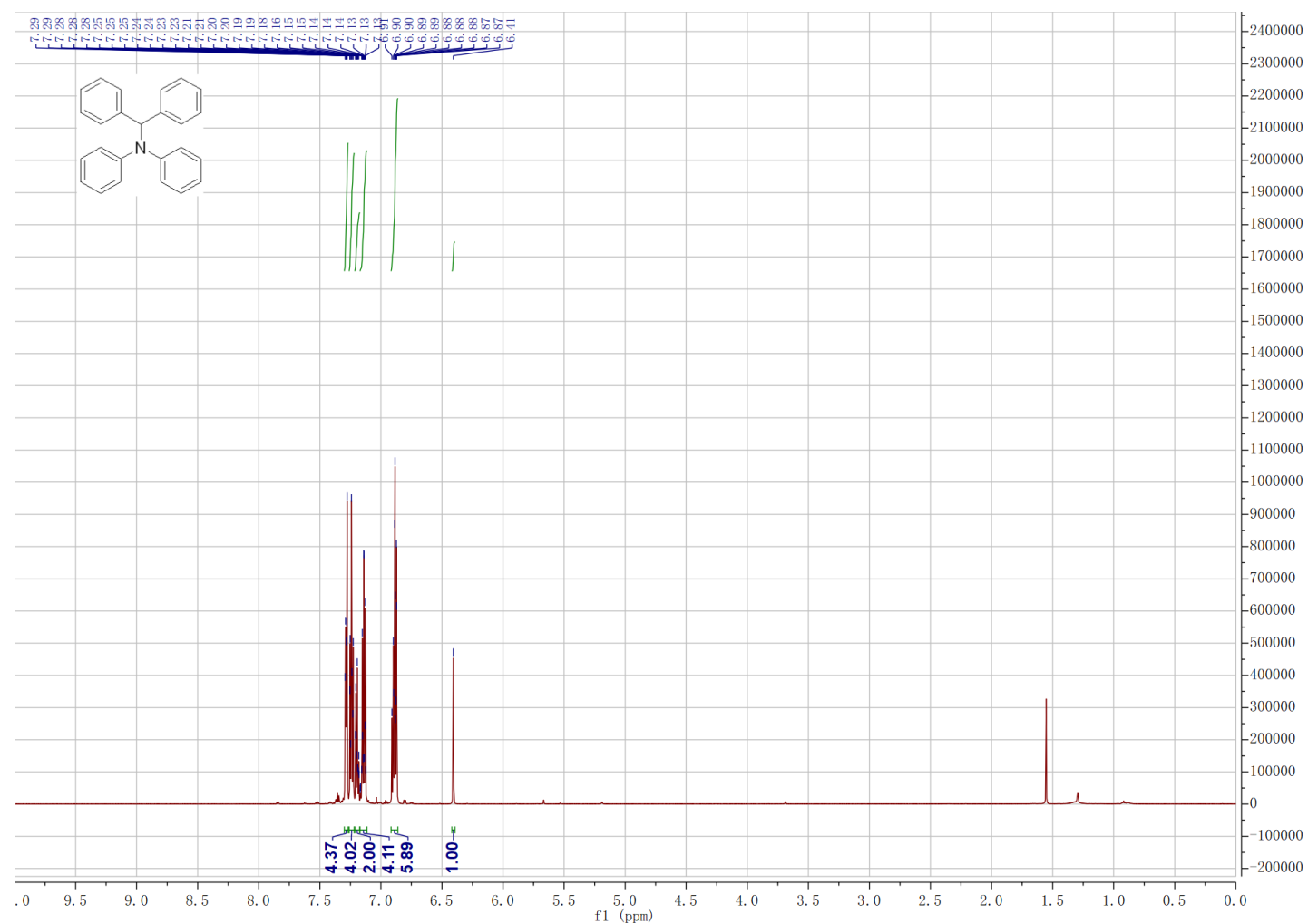

${ }^{13} \mathrm{C}$ NMR (151 MHz, Chloroform- $d$ )

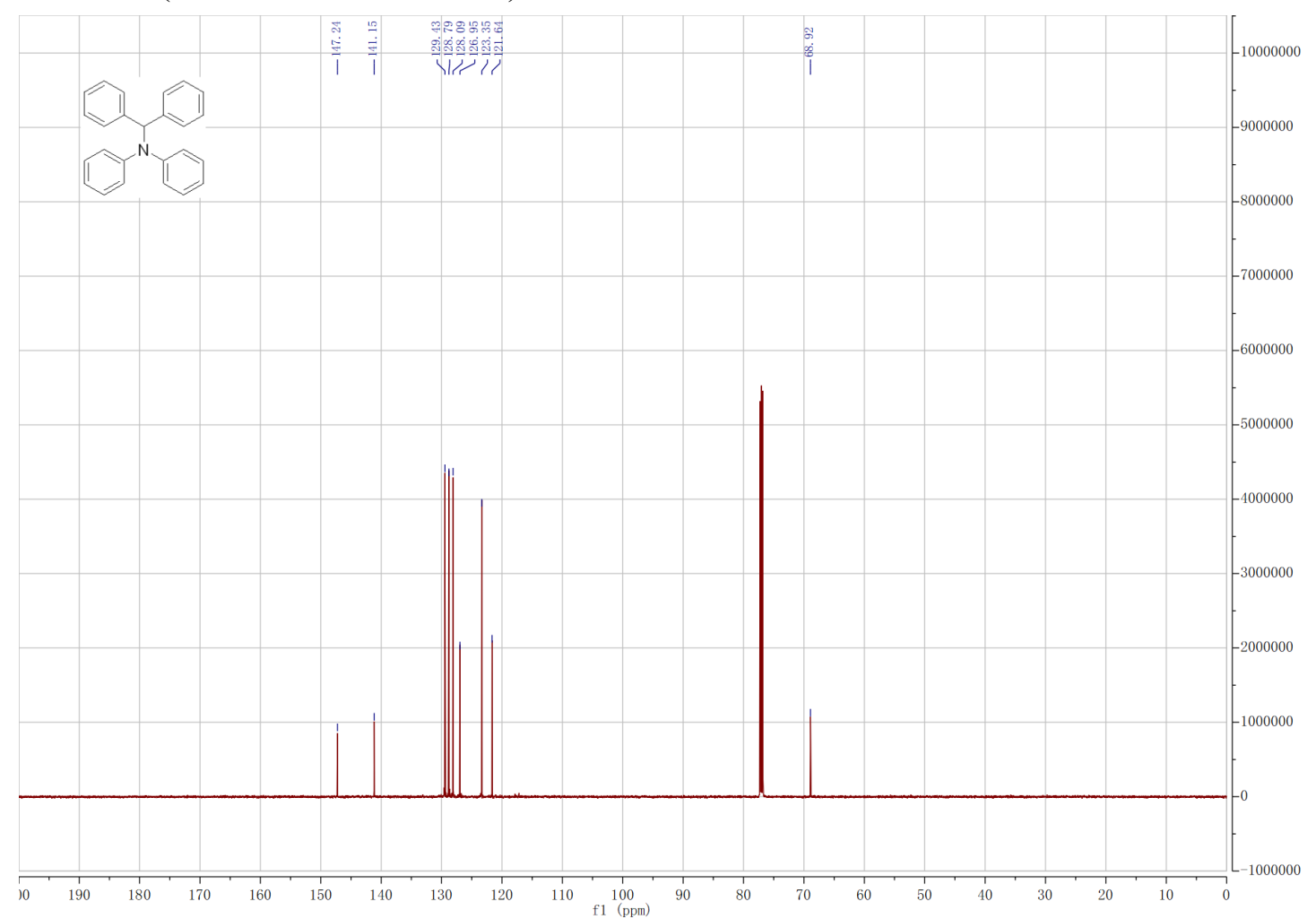

